\title{
Segregation von Wasserstoff und Deuterium an Versetzungen in Palladium
}

\author{
Dissertation \\ zur Erlangung des Doktorgrades \\ der Mathematisch-Naturwissenschaftlichen Fakultäten \\ der Georg-August-Universität zu Göttingen
}

vorgelegt von

Michael Helmut Maxelon

aus Kassel

Göttingen 2000 
D 7

Referent:

Prof. Dr. R. Kirchheim

Korreferent:

Prof. Dr. W. Felsch

Tag der mündlichen Prüfung:

26. April 2000 


\section{Inhaltsverzeichnis}

1 EINLEITUNG

2 DIE WECHSELWIRKUNG VON WASSERSTOFF MIT VERSETZUNGEN

2.1 Wasserstoff in Palladium

2.2 Grundlagen zu Wasserstoff in Metallen

2.3 Das elastische Feld einer Versetzung 9

2.3.1 Grundlagen der linearen Elastizitätstheorie $\quad 10$

2.3.2 Das Spannungsfeld einer Schraubenversetzung 11

2.3.3 Das Spannungsfeld einer Stufenversetzung 12

2.3.4 Der hydrostatische Druck 13

$\begin{array}{lll}2.4 & \text { Modell zur Segregation von Wasserstoff an Versetzungen } & 15\end{array}$

3 UNTERSUCHUNG DES SEGREGATIONSGEBIETES MIT NEUTRONENSTREUUNG $\quad 20$

$\begin{array}{lll}3.1 & \text { Grundlagen der Neutronenstreuung } & 20\end{array}$

3.2 Typische Streukurven ausgewählter Streuobjekte 23

3.2.1 Guinierverhalten 23

$\begin{array}{lll}3.2 .2 & \text { Porodverhalten } & 24\end{array}$

3.3 Das Streuverhalten des Segregationszylinders $\quad 24$

4 BESCHREIBUNG DER EXPERIMENTE 29

4.1 Probenpräparation 29

4.1.1 Elektrochemische Beladung 29

4.1.2 Versetzungsbildung durch Phasenseparation 31

4.1.3 Charakterisierung der Versetzungsdichte 32

4.2 Bestimmung der segregierten Wasserstoffmenge 35

4.2.1 Die Methode der Gasvolumetrie 36

4.2.2 Isothermen für Wasserstoff und Deuterium 38 
4.3.1 Experimentelles Vorgehen $\quad 41$

4.3.2 Berechnung des makroskopischen Streuquerschnittes 43

$5 \quad$ ERGEBNISSE UND DISKUSSION $\quad 47$

$\begin{array}{llr}5.1 & \text { Gasvolumetrische Messungen } & 47\end{array}$

$\begin{array}{llr}5.2 & 50\end{array}$

5.2.1 Zylinderradius in Abhängigkeit von der Wasserstoffkonzentration 51

$\begin{array}{ll}\text { 5.2.2 Streuverhalten deuterierter Proben } & 59\end{array}$

$\begin{array}{lll}\text { 5.2.3 Porodverhalten } & 63\end{array}$

6 ZUSAMMENFASSUNG $\quad 65$

$\begin{array}{ll}\text { LITERATURVERZEICHNIS } & 67\end{array}$

$\begin{array}{ll}\text { LEBENSLAUF } & 69\end{array}$ 


\section{Einleitung}

Wasserstoff gilt derzeit als Energieträger der Zukunft. Die ersten Brennstoffzellen erzeugen sowohl im stationären als auch im mobilen Einsatz Strom und wiederaufladbare Batterien auf der Basis von Metall-Wasserstoffsystemen haben die Serienreife erreicht. Das Interesse an Materialien, die Wasserstoff enthalten, ist außerdem durch die smart properties motiviert, die einige Materialien in Abhängigkeit von ihrer Wasserstoffkonzentration aufweisen. Hierbei sind beispielsweise schaltbare Spiegel zu nennen, die durch eine Änderung der Wasserstoffkonzentration von metallisch glänzend auf transparent schalten [Gri97]. Auch der hohe Wirkungsgrad neuer Solarzellen wird durch den gezielten Einsatz von Wasserstoff erreicht.

Seit Graham 1866 [Gra66] entdeckte, dass Palladium große Mengen Wasserstoff aufnimmt, sind Metall-Wasserstoffsysteme Gegenstand intensiver Forschung. Sie streben mit einer für Metalle bei tiefen Temperaturen außergewöhnlich hohen Kinetik in das thermodynamische Gleichgewicht. Mit Wasserstoff als Sonde lassen sich daher Zustände des Metalls bei niedrigen Temperaturen untersuchen, die bei höheren Temperaturen nicht stabil wären. Dabei wird die Wechselwirkung von Wasserstoff mit Defekten ausgenutzt [Kir88], insbesondere seine Anreicherung an Versetzungen.

Die Wechselwirkung von Zwischengitteratomen mit Versetzungen wurde erstmals von Cottrell und Bilby [Cot49] beschrieben. Die Autoren schlossen aus der Zeitabhängigkeit der Fließspannung bei niedriglegierten Stählen auf eine Anreicherung des Kohlenstoffes an den Versetzungen („Cottrell-Wolke“). Die treibende Kraft ist dabei eine Absenkung der elastischen Energie eines Zwischengitteratomes (oder eines größeren substitutionellen Fremdatomes) durch Diffusion in einen aufgeweiteten Gitterbereich, wie er durch eine Stufenversetzung unterhalb der eingeschobenen Halbebene verursacht wird. Für Sauerstoff in Silizium konnte eine derartige Anreicherung an Versetzungen mit hochauflösender Elektronenmikroskopie direkt nachgewiesen werden [Bou82]. Neueste Untersuchungen von Bor in EisenAluminium mit der tomographischen Atomsonde zeigen einen Schlauch mit 50 Å Durchmesser tangential zur Versetzungslinie, in dem die Konzentration von Bor auf das fünfzigfache des Matrixwertes erhöht ist [Bla99]. Auf die Segregation von Wasserstoff an Versetzungen konnte indirekt aus Messungen des elektrischen Widerstandes geschlossen werden, da der an den Versetzungen gebundene Wasserstoff im Vergleich zum interstitiell gelösten kaum zur Streuung der Elektronenwelle beiträgt [Rod83, Szö87]. Messungen der elektromotorischen Kraft lassen auf eine Zunahme der segregierten Wasserstoffmenge mit der Konzentration schließen [Kir88]. Anreicherungen von Deuterium an Versetzungen wurden erstmals von Kirchheim als räumlich ausgedehnte Streulängendichteänderungen mit der Kleinwinkelneutronenstreuung nachgewiesen [Kir88]. Heuser und King fanden in entsprechenden Messungen deuteriumreiche Schläuche in Palladium mit einem Durchmesser von $22 \AA$ bei einer Deuteriumkonzentration von $5,5 \cdot 10^{-3} \mathrm{D} / \mathrm{Pd}$ [Heu91, Heu97]. 
Die radiale Ausdehung des Segregationsgebietes wird in der vorliegenden Arbeit erstmals auch für Wasserstoff mit der Methode der Kleinwinkelneutronenstreuung untersucht. Ziel der Untersuchungen ist es, die Segregation an Versetzungen in Palladium in Abhängigkeit von der Wasserstoffkonzentration zu charakterisieren. Die Ergebnisse werden in einem thermodynamischen Modell beschrieben. Die Streulängendifferenz von Wasserstoff und Deuterium führt zu einem unterschiedlichen Streukontrast. Ein Vergleich der Kleinwinkelstreuung von deuterierten und hydrierten Proben ermöglicht es daher, die Aufweitung des Segregationsgebietes im Palladiumgitter zu bestimmen. Eine weitgehend vollständige Beschreibung des Problems gelingt durch den Einsatz zusätzlicher Methoden. Mittels der Elektronenmikroskopie kann die für die Größe des Segregationseffektes entscheidende Versetzungsdichte ermittelt werden. Gasvolumetrische Messungen kommen als unabhängige Methode zur Untersuchung der Löslichkeit von Wasserstoff und Deuterium zum Einsatz und bestätigen die Interpretation der Streuergebnisse.

In Kapitel 2 erfolgt zunächst eine thermodynamische Beschreibung der Segregation von Wasserstoff an Versetzungen. Anschließend wird in Kapitel 3 das Streuverhalten des Segregationsgebietes berechnet und im Verlauf der Arbeit mit den experimentellen Ergebnissen der Kleinwinkelneutronenstreuung verglichen. Die Beschreibung der Experimente erfolgt in Kapitel 4, in dem auch die Ergebnisse der Elektronenmikroskopie, der Gasvolumetrie und teilweise auch die der Neutronenstreuung vorgestellt werden. Letztere werden in Kapitel 5 zusammengefasst, dargestellt und vergleichend diskutiert. Die physikalische Interpretation der Messergebnisse gelingt mit den vorgestellten theoretischen Modellen. Die in dieser Arbeit gewonnen Erkenntnisse fasst Kapitel 6 zusammen. 


\section{Die Wechselwirkung von Wasserstoff mit Versetzungen}

In diesem Kapitel wird das Segregationsverhalten von Wasserstoff an Versetzungen thermodynamisch beschrieben. Ausgehend von dem elastischen Feld einer Stufenversetzung wird ein einfaches Modell entwickelt, welches die radiale Ausdehnung des zylinderförmigen Segregationsgebietes mit experimentell bestimmbaren Größen verknüpft. Einleitend werden die Grundlagen der interstitiellen Lösung von Wasserstoff in Metallen dargestellt und das System Palladium-Wasserstoff als Modellsystem eingeführt.

\subsection{Wasserstoff in Palladium}

Seit Graham 1866 [Gra66] entdeckte, dass Palladium große Mengen Wasserstoff aufnimmt, gilt das System Palladium-Wasserstoff als physikalisches Modellsystem für das Verhalten von Wasserstoff in Metallen. Eine Übersicht der umfangreichen experimentellen Daten findet sich beispielsweise in [Wic78, Oat81, Fla91].
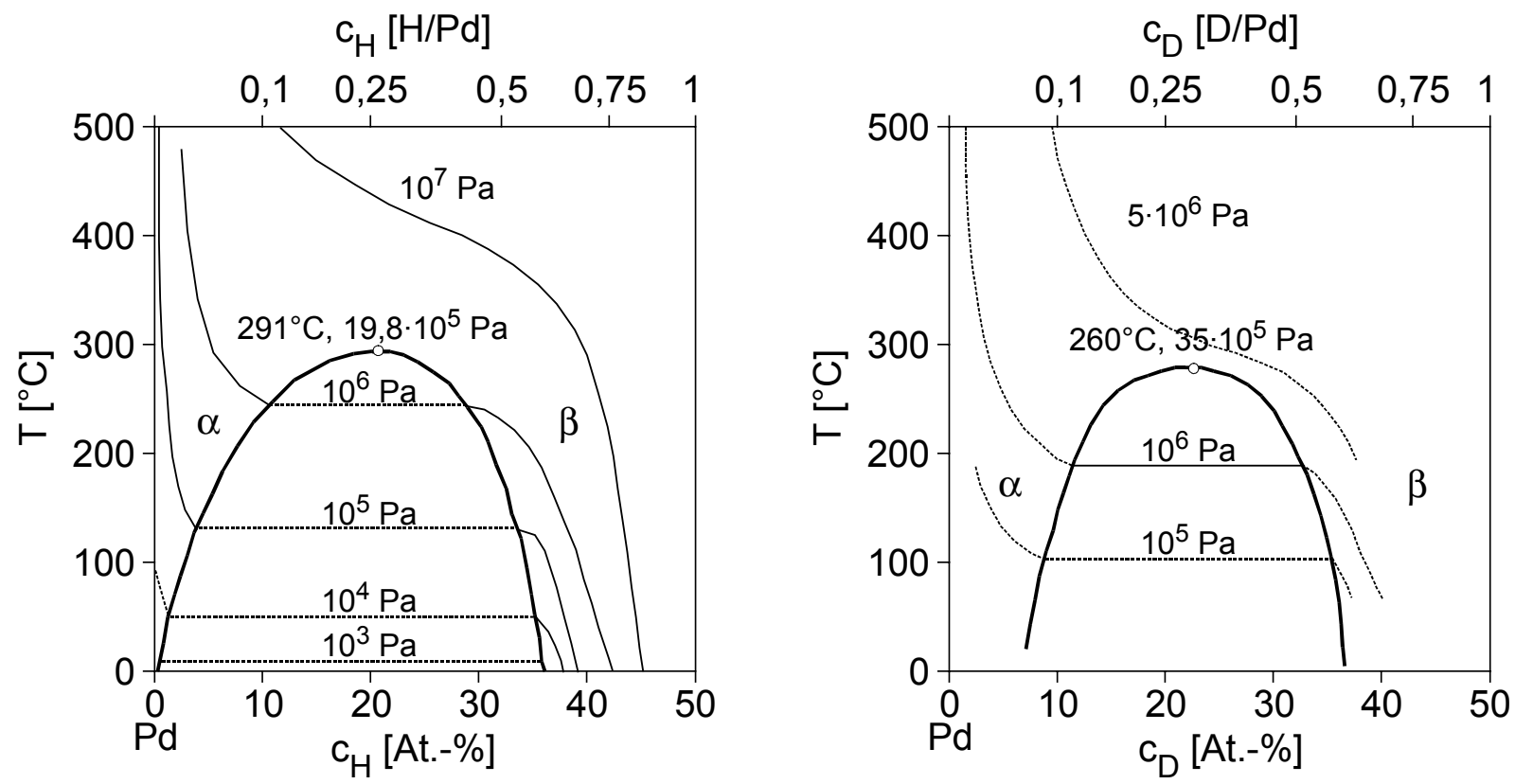

Abbildung 2.1: $\quad$ Phasendiagramme der Systeme Palladium-Wasserstoff und Palladium-Deuterium. Für verschiedene Gleichgewichtsgasdrücke sind die Isobaren angegeben [Rau76].

Tabelle 2.1: $\quad$ Kritische Temperatur $T_{c}$ und kritischer Druck $p_{c}$ für die Systeme Palladium-Wasserstoff und Palladium-Deuterium [Rau76]

\begin{tabular}{|c|c|c|}
\hline & $\mathrm{T}_{\mathrm{C}}$ & $\mathrm{p}_{\mathrm{C}}$ \\
\hline Palladium-Wasserstoff & $564 \mathrm{~K}$ & $19,8 \cdot 10^{5} \mathrm{~Pa}$ \\
\hline Palladium-Deuterium & $533 \mathrm{~K}$ & $35 \cdot 10^{5} \mathrm{~Pa}$ \\
\hline
\end{tabular}


Die Oktaederplätze eines kubisch flächenzentrierten Gitters bilden selbst ein um 1/2[100] verschobenes kfz-Gitter (Abb. 2.2), das durch den Wasserstoff stochastisch besetzt wird. Es existiert ein Zwischengitterplatz pro Palladiumatom. Ordnungsphasen sind ebenso wie eine Besetzung der Tetraederplätze nur für tiefe Temperaturen bekannt, bei denen in dieser Arbeit keine Messungen durchgeführt worden sind. Bei höheren Temperaturen bildet das System Palladium-Wasserstoff (Abb. 2.1) zwei Phasen ( $\alpha$ und $\beta^{1}$ ), die beide eine kubisch flächenzentrierte Gitterstruktur besitzen. Die Besetzung der interstitiellen Gitterplätze mit Wasserstoff oder Deuterium führt zu einer Gitteraufweitung, die Peisl mit 2,9 $\AA^{3}$ pro Atom angibt [Pei78]. Die $\beta$-Phase exitiert bei höheren Konzentrationen aufgrund der Wasserstoff-WasserstoffWechselwirkung mit einem Volumenmisfit von ca. $16 \%$ zur $\alpha$-Phase. Unterhalb von $\mathrm{T}_{\mathrm{c}}$ (vgl. Tabelle 2.1) tritt zwischen der $\alpha$ - und $\beta$-Phase eine Mischungslücke auf. Das Zweiphasengebiet erstreckt sich bei Raumtemperatur von $0,008 \mathrm{H} / \mathrm{Pd}$ bis $0,607 \mathrm{H} / \mathrm{Pd}$ [Vö178]. Für Deuterium ist die maximale Löslichkeit in der $\alpha$-Phase größer, wie Abbildung 2.1 zeigt. Besonders bemerkenswert ist der höhere Gleichgewichtsgasdruck für das schwerere Isotop Deuterium im Vergleich zu Wasserstoff bei gegebener Temperatur und Konzentration. Dieser Isotopeneffekte wird in Kapitel 4.2 diskutiert. Außerdem ist die kritische Temperatur für das System $\mathrm{Pd}-\mathrm{D}_{2}$ kleiner als im Fall des $\mathrm{Pd}-\mathrm{H}_{2}$.

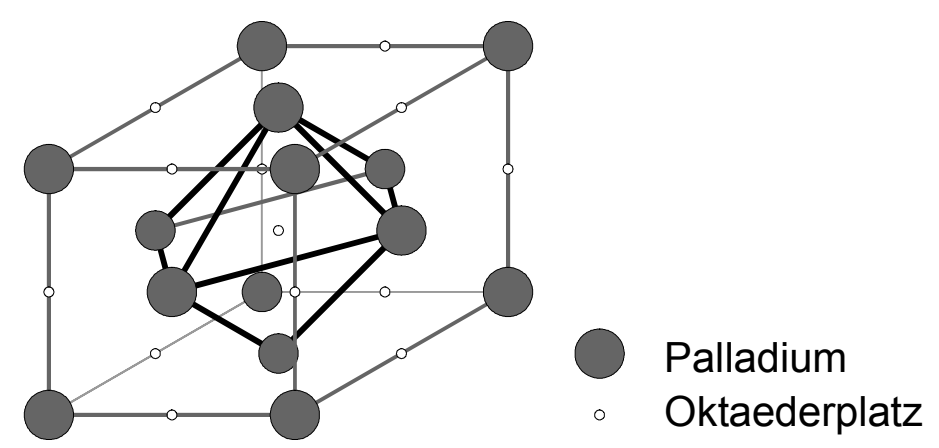

Abbildung 2.2: Okteaderlücke des kubisch flächenzentrierten Gitters. Die Oktaederplätze bilden selbst ein um $1 / 2[100]$ verschobenes kfz-Gitter.

\footnotetext{
${ }^{1}$ Beide Phasen $\alpha$ und $\beta$ in den Systemen Palladium-Wasserstoff und Palladium-Deuterium werden thermodynamisch durch eine gemeinsame Kurve der freien Energie beschrieben. Der Konvention entsprechend wäre die Hochkonzentrationsphase daher $\alpha^{6}$-Phase zu nennen. In dieser Arbeit wird jedoch die übliche und historisch begründete Bezeichnung $\beta$-Phase verwendet.
} 


\subsection{Grundlagen zu Wasserstoff in Metallen}

Der Wasserstoff besetzt die Plätze des Zwischengitters nach der Fermi-Dirac-Statistik [Bes58]. Diese berücksichtigt eine maximale Belegung jedes Zwischengitterplatzes durch nur ein Atom. Den Plätzen kann eine Bindungsenergie $E_{i}$ zugeordnet werden, so dass man eine Platzenergieverteilungsfunktion $\mathrm{n}(\mathrm{E})$ erhält [Kir81b, Kir88]. Das Integral über diese Zustandsdichte multipliziert mit der Besetzung ergibt die Konzentration c, die als das Verhältnis der Anzahl der Wasserstoffatome und der Anzahl der Plätze definiert ist.

$$
c=\int_{-\infty}^{\infty} \frac{n(E) d E}{1+\exp \left(\frac{E-\mu}{R T}\right)}
$$

Für den Fall eines idealen Gitters, bei dem alle Plätze dieselbe Energie $\mathrm{E}_{0}$ besitzen, entspricht sie der Diracschen Deltafunktion $\delta\left(E_{-} E^{0}\right)$. Nahe einer Stufenversetzung ist die Platzenergie im aufgeweiteten Gitter (Dilatationsgebiet) abgesenkt und im Kompressionsfeld angehoben. Abbildung 2.3 zeigt die entsprechenden Potentialverläufe und die sich daraus ergebenden Platzenergieverteilungsfunktionen [Kir88].

(1)
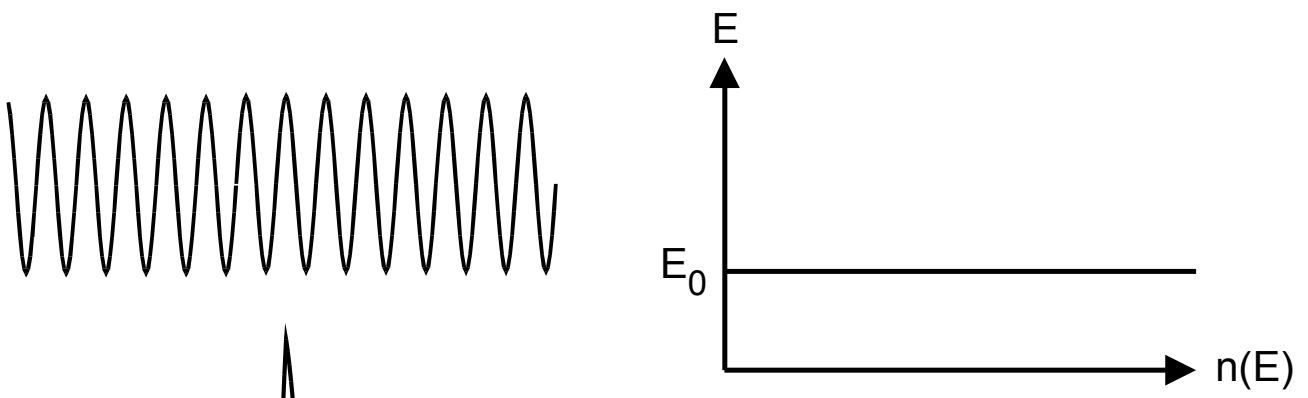

(2)
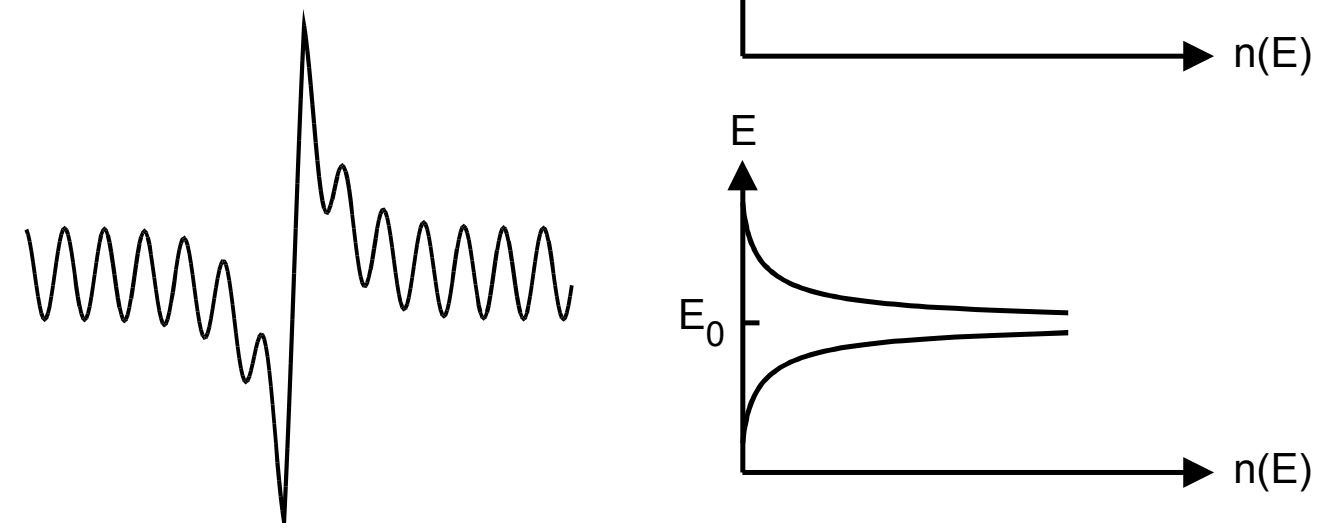

Abbildung 2.3: Potentialverläufe (1) eines idealen Gitters und (2) einer Stufenversetzung mit den zugehörigen Platzenergieverteilungsfunktionen n(E). 
Für versetzungsreiches Material müssen zwei Beiträge der Platzenergieverteilungsfunktion $\mathrm{n}(\mathrm{E})$ betrachtet werden. Dazu wird die Gesamtzahl $\mathrm{M}$ der Zwischengitterplätze eingeteilt in die des ungestörten Gitters $\mathrm{M}_{\mathrm{f}}$ und die durch die Versetzung gestörten Gitterplätze $\mathrm{M}_{\mathrm{s}}$. Daraus ergeben sich die Anteile $n_{\alpha}^{0}=M_{\alpha} / M(\alpha=f \vee s)$.

$$
\begin{array}{ll}
\mathrm{n}_{\mathrm{f}}(\mathrm{E})=\mathrm{n}_{\mathrm{f}}^{0} \cdot \delta\left(E-\mathrm{E}_{0}\right) & \text { ideales Gitter } \\
\mathrm{n}_{\mathrm{s}}(\mathrm{E}) \propto \mathrm{n}_{\mathrm{s}}^{0} \cdot\left(\frac{\mathrm{Eb} \bar{V}_{\mathrm{H}}}{6 \pi(1-v)}\right)^{2} \cdot \frac{\pi \zeta}{2 \mathrm{E}^{3}} & \text { Stufenversetzung }
\end{array}
$$

$\mathrm{E}_{0}$ ist hierbei die Platzenergie des ungestörten Gitters. Gleichung 2.2 enthält die Versetzungsdichte $\zeta$ des Materials und den Wechselwirkungsterm aus Gleichung 2.25 [Kir88], der die Wechselwirkung der Zwischengitteratome mit dem elastischen Feld der Versetzung beschreibt und in Kapitel 2.4 ausführlich erläutert wird. Die sich aus den Platzenergieverteilungen ergebenden Integrale werden in [Kir83] diskutiert. Im Fall der Stufenversetzung existiert keine geschlossene Lösung. In der vorliegenden Arbeit ist diese entbehrlich und es wird folgende einfache Unterteilung verwendet:

$$
\begin{aligned}
c & =\int_{-\infty}^{\infty} \frac{n_{f}(E) d E}{1+\exp \left(\frac{E-\mu}{R T}\right)}+\int_{-\infty}^{\infty} \frac{n_{s}(E) d E}{1+\exp \left(\frac{E-\mu}{R T}\right)} \\
& =\frac{n_{f}^{0}}{1+\exp \left(\frac{E^{0}-\mu}{R T}\right)}+c_{s} \\
& =c_{f}+c_{s}
\end{aligned}
$$

Für das kfz-Gitter des Palladiums mit je einem Zwischengitterplatz pro Palladiumatom entspricht diese Konzentrationsangabe dem Wasserstoff-zu-Metallatomverhältnis H/Pd. Gleichung 2.3 enthält drei wesentliche Ergebnisse. Zum einen ergibt sich die Gesamtkonzentration c als Summe der partiellen Konzentrationen $c_{\mathrm{f}}$ des ,freien“"Wasserstoffs im idealen Gitter und $\mathrm{c}_{\mathrm{s}}$ im Spannungsfeld der Versetzung. Außerdem werden die energetisch begünstigten Plätze im Dilatationsfeld der Versetzung bevorzugt besetzt. In Kapitel 2.4 wird begründet, dass das Kompressionsfeld für die vorliegende Arbeit ohne Bedeutung ist.

Schließlich folgt aus dem Ausdruck für $\mathrm{c}_{\mathrm{f}}$ direkt das chemische Potential im Modell der idealen Lösung unter Verwendung des Standardpotential $\mu_{\mathrm{H}}^{0}$ :

$$
\mu=\mu_{\mathrm{H}}^{0}+\mathrm{RT} \cdot \ln \left(\frac{\mathrm{c}_{\mathrm{f}}}{1-\mathrm{c}_{\mathrm{f}}}\right)
$$


Diese Gleichung ist für die $\alpha$-Phase des Palladiums gültig, die mit einer maximalen Löslichkeit von $0,008 \mathrm{H} / \mathrm{Pd}$ (bei Raumtemperatur) als ideal verdünnt angesehen werden kann. Der oben beschriebene Einfluß von Versetzungen wird dabei ebensowenig berücksichtigt wie eine Wechselwirkung der Wasserstoffatome untereinander.

Im thermodynamischen Gleichgewicht befindet sich der interstitiell gelöste Wasserstoff im Gleichgewicht mit der Gasphase, die durch den Wasserstoffpartialdruck $\mathrm{p}_{\mathrm{H}_{2}}$ beschrieben wird.

$$
\mu=\frac{1}{2} \mu_{\mathrm{H}_{2}}^{0}+\frac{\mathrm{RT}}{2} \cdot \ln \left(\mathrm{p}_{\mathrm{H}_{2}}\right)
$$

Aus den Gleichungen 2.4 und 2.5 folgt

$$
\ln \left(\sqrt{\mathrm{p}_{\mathrm{H}_{2}}}\right)=\ln \left(\frac{\mathrm{c}_{\mathrm{f}}}{1-\mathrm{c}_{\mathrm{f}}}\right)+\frac{\mu_{\mathrm{H}}^{0}-\frac{1}{2} \mu_{\mathrm{H}_{2}}^{0}}{\mathrm{RT}}
$$

und damit das Sievertssche Gesetz [Sie29] für die ideal verdünnte Lösung $\left(\mathrm{c}_{\mathrm{f}} \ll 1\right)$

$$
\mathrm{c}_{\mathrm{f}}=\mathrm{K} \sqrt{\mathrm{p}_{\mathrm{H}_{2}}} \quad \text { mit } \mathrm{K}=\exp \left(\frac{\mu_{\mathrm{H}}^{0}-\frac{1}{2} \mu_{\mathrm{H}_{2}}^{0}}{\mathrm{RT}}\right) \text {, }
$$

der Sievertskonstanten, deren Temperaturabhängigkeit aus der Desorptionsenthalpie folgt. Das Sievertssche Gesetz entspricht dem Henryschen Gesetz mit Berücksichtigung der Dissoziation des $\mathrm{H}_{2}$-Moleküls.

Für höhere Konzentrationen sind insbesondere elastische und elektronische Wechselwirkungen zu berücksichtigen [Wic78, Bro72]. Sie sind Ursache der $\beta$-Phase und können als ein Beitrag $\Delta \mu_{\mathrm{H}}$ zum chemischen Potential zusammengefaßt werden:

$$
\mu=\mu_{\mathrm{H}}^{0}+\mathrm{RT} \cdot \ln \left(\frac{\mathrm{c}}{1-\mathrm{c}}\right)+\Delta \mu_{\mathrm{H}}
$$

Die Wechselwirkung von Wasserstoff mit Versetzungen kann durch einen äquivalenten Term beschrieben werden. Ein entsprechendes Modell wird in Kapitel 2.4 vorgestellt, welches die Wechselwirkung eines Zwischengitteratoms mit Versetzungen rein elastisch berücksichtigt.

\subsection{Das elastische Feld einer Versetzung}

In dieser Arbeit lassen sich die beobachteten Effekte erfolgreich durch eine elastische Wechselwirkung des interstitiell gelösten Wasserstoff mit den Versetzungen beschreiben. 
Elektronische Beiträge können zumindest in der $\alpha$-Phase des Palladiumhydrids vernachlässigt werden.

Für die Bestimmung der elastischen Wechselwirkung durch den hydrostatischen Druck werden die Spannungsfelder einer Stufen- und Schraubenversetzung berechnet. Kapitel 2.3.1 dient zunächst als Einführung in die Elastizitätstheorie.

\subsubsection{Grundlagen der linearen Elastizitätstheorie}

In der linearen Elastizitätstheorie gilt das verallgemeinerte Hooksche Gesetz zwischen dem Spannungstensor $\sigma_{\mathrm{ij}}$ und dem Verzerrungstensor $\varepsilon_{\mathrm{k} 1}$ :

$$
\sigma_{\mathrm{ij}}=\mathrm{C}_{\mathrm{ijkl}} \cdot \varepsilon_{\mathrm{kl}}
$$

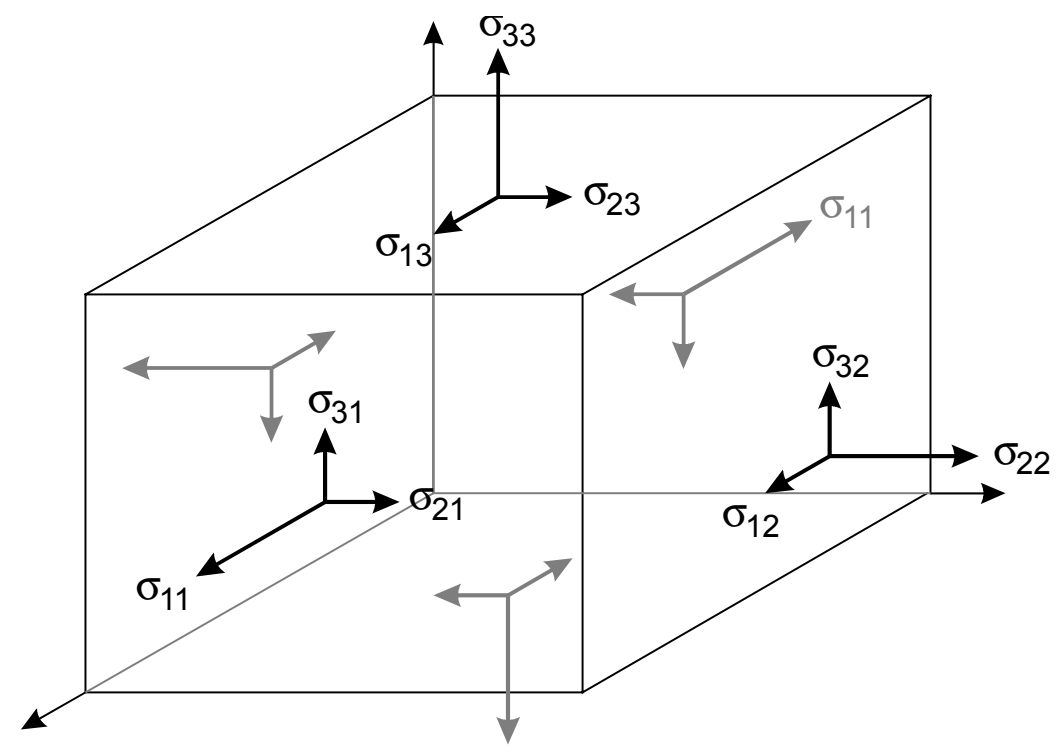

Abbildung 2.4: Die Spannungskomponenten $\sigma_{\mathrm{ij}}$ an einem infinitesimalen Volumenelement. Die Spannung wirkt in i-Richtung auf die Fläche mit Normale j.

Der Verzerrungstensor $\varepsilon_{\mathrm{kl}}$ ist gegeben durch die Verschiebungen $\overrightarrow{\mathrm{u}}(\overrightarrow{\mathrm{x}})$ des Kristalls:

$$
\varepsilon_{\mathrm{kl}}=\frac{1}{2}\left(\frac{\partial \mathrm{u}_{\mathrm{k}}}{\partial \mathrm{x}_{1}}+\frac{\partial \mathrm{u}_{1}}{\partial \mathrm{x}_{\mathrm{k}}}\right)
$$

Die Materialeigenschaften bestimmen den Tensor $\mathrm{C}_{\mathrm{ijk}}$ der Elastizitätsmoduln. Es handelt sich um einen Tensor 4. Stufe mit 81 elastischen Konstanten, die sich im vorliegenden Fall eines kubischen Gitters durch Symmetrieüberlegungen auf drei unabhängige Konstanten reduzieren lassen. Aufgrund der Symmetrie des Verzerrungstensor $\varepsilon_{\mathrm{k} l}$ kann $\mathrm{C}_{\mathrm{ijkl}}$ durch 21 Moduln dargestellt werden, von denen beim kubischen Gitter nur drei unabhängig sind. Palladium ist au- 
ßerdem isotrop und wird deshalb durch die beiden Laméschen Konstanten $\lambda$ und $\mu$ vollstandig beschrieben. In Voigtscher Notation ( $\mathrm{xx} \rightarrow 1$, yy $\rightarrow 2$, $\mathrm{zz} \rightarrow 3$, $\mathrm{xy} \rightarrow 4$, $\mathrm{xz} \rightarrow 5, \mathrm{yz} \rightarrow 6$ ) wird dem Tensor $\mathrm{C}_{\mathrm{ijk}}$ der Elastizitätsmoduln eine 6×6-Matrix zugeordnet [Hir68]:

$$
\mathrm{C}_{\alpha \beta}=\left|\begin{array}{cccccc}
\lambda+2 \mu & \lambda & \lambda & 0 & 0 & 0 \\
\lambda & \lambda+2 \mu & \lambda & 0 & 0 & 0 \\
\lambda & \lambda & \lambda+2 \mu & 0 & 0 & 0 \\
0 & 0 & 0 & 2 \mu & 0 & 0 \\
0 & 0 & 0 & 0 & 2 \mu & 0 \\
0 & 0 & 0 & 0 & 0 & 2 \mu
\end{array}\right|
$$

Zwischen der Laméschen Konstanten $\lambda$, dem Schermodul $\mu$, dem Schubmodul G, dem ElastizitätsmodulE und der Poissonschen Querkontraktionszahl $v$ bestehen folgende Zusammenhänge:

$$
\begin{aligned}
v & =\frac{\lambda}{2 \lambda+2 \mu} \\
\mathrm{G} & =\mu \\
\mathrm{E} & =2 \mu\left(\frac{\lambda}{2 \lambda+2 \mu}+1\right)=2 \mathrm{G}(1+v)
\end{aligned}
$$

Damit ist es möglich, das Spannungsfeld $\sigma$ aus den Verschiebungen $\overrightarrow{\mathrm{u}}(\overrightarrow{\mathrm{x}})$ der Gitteratome um eine Versetzung unter Verwendung der experimentell zugänglicher Größen $v$ und $\mathrm{E}$ zu berechnen.

\subsubsection{Das Spannungsfeld einer Schraubenversetzung}

Eine Schraubenversetzung wird durch das Bild einer Wendeltreppe beschrieben. Die Verschiebung existiert ausschließlich in z-Richtung und ist in Zylinderkoordinaten nur abhängig vom Winkel $\vartheta$ :

$$
\begin{aligned}
& \mathrm{u}_{\mathrm{x}}=\mathrm{u}_{\mathrm{y}}=0 \\
& \mathrm{u}_{\mathrm{z}}=\frac{\mathrm{b} \vartheta}{2 \pi}=\frac{\mathrm{b}}{2 \pi} \arctan \left(\frac{\mathrm{y}}{\mathrm{x}}\right)
\end{aligned}
$$


Dabei bezeichnet $\mathrm{b}$ den Burgersvektor, um den die Versetzung den Kristall bei einem Burgersumlauf verschiebt. Mit der Definition der Verzerrung $\varepsilon$ (Gl. 2.10) folgt aus den Verschiebungen:

$$
\begin{aligned}
& \varepsilon_{\mathrm{xx}}=\varepsilon_{\mathrm{yy}}=\varepsilon_{\mathrm{zz}}=\varepsilon_{\mathrm{xy}}=\varepsilon_{\mathrm{yx}}=0 \\
& \varepsilon_{\mathrm{xz}}=\varepsilon_{\mathrm{zx}}=-\frac{\mathrm{b}}{4 \pi} \frac{\mathrm{y}}{\mathrm{x}^{2}+\mathrm{y}^{2}} \\
& \varepsilon_{\mathrm{yz}}=\varepsilon_{\mathrm{zy}}=\frac{\mathrm{b}}{4 \pi} \frac{\mathrm{x}}{\mathrm{x}^{2}+\mathrm{y}^{2}}
\end{aligned}
$$

Unter Verwendung des Hookschen Gesetzes und des oben angegeben Tensors der elastischen Konstanten (Gl. 2.9, 2.11, 2.12) erhält man das Spannungsfeld einer Schraubenversetzung:

$$
\begin{aligned}
& \sigma_{\mathrm{xx}}=\sigma_{\mathrm{yy}}=\sigma_{\mathrm{zz}}=\sigma_{\mathrm{xy}}=\sigma_{\mathrm{yx}}=0 \\
& \sigma_{\mathrm{xz}}=\sigma_{\mathrm{zx}}=-\frac{\mathrm{Gb}}{2 \pi} \frac{\mathrm{y}}{\mathrm{x}^{2}+\mathrm{y} 2} \\
& \sigma_{\mathrm{yz}}=\sigma_{\mathrm{zy}}=\frac{\mathrm{Gb}}{2 \pi} \frac{\mathrm{x}}{\mathrm{x}^{2}+\mathrm{y} 2}
\end{aligned}
$$

Folglich bewirkt eine Schraubenversetzung in einem isotropen Medium ausschließlich Scherspannungen.

\subsubsection{Das Spannungsfeld einer Stufenversetzung}

Die Verschiebung $\overrightarrow{\mathrm{u}}(\overrightarrow{\mathrm{x}})$ um eine unendlich lange Stufenversetzung besitzt keinen Beitrag in Richtung der Versetzungslinie $\left(\mathrm{u}_{\mathrm{z}}=0\right)$. Die Lösungen in der Ebene sind nicht trivial und das Ergebnis wird der Literatur entnommen [Cot64]:

$$
\begin{aligned}
& \sigma_{\mathrm{xx}}=-\frac{\mathrm{Gb}}{2 \pi(1-v)} \cdot \frac{3 \mathrm{x}^{2}+\mathrm{y}^{2}}{\left(\mathrm{x}^{2}+\mathrm{y}^{2}\right)^{2}} \cdot \mathrm{y} \\
& \sigma_{\mathrm{yy}}=\frac{\mathrm{Gb}}{2 \pi(1-v)} \cdot \frac{\mathrm{x}^{2}-\mathrm{y}^{2}}{\left(\mathrm{x}^{2}+\mathrm{y}^{2}\right)^{2}} \cdot \mathrm{y} \\
& \sigma_{\mathrm{zz}}=v \cdot\left(\sigma_{\mathrm{xx}}+\sigma_{\mathrm{yy}}\right) \\
& \sigma_{\mathrm{xy}}=\sigma_{\mathrm{yx}}=\frac{\mathrm{Gb}}{2 \pi(1-v)} \cdot \frac{\mathrm{x}^{2}-\mathrm{y}^{2}}{\left(\mathrm{x}^{2}+\mathrm{y}^{2}\right)^{2}} \cdot \mathrm{x}
\end{aligned}
$$


Die Stufenversetzung besitzt neben der Scherspannung $\sigma_{\mathrm{xy}}$ auch eine Spannung, die auf das Flächenelement normal wirkt (vgl. Abb. 2.4). Bei negativem Vorzeichen spricht man üblicherweise von einer Kompressionsspannung und bei positivem Vorzeichen von einer Dilatationsspannung.

\subsubsection{Der hydrostatische Druck}

Ein Zwischengitteratom leistet aufgrund seines partiellen Atomvolumens $\bar{V}$ einen Beitrag $\Delta \mathrm{G}=\sigma_{\mathrm{h}} \cdot \overline{\mathrm{V}}_{\mathrm{H}}$ zur freien Enthalpie des Systems. Unter dem hydrostatischen Druck $\sigma_{\mathrm{h}}$ versteht man den effektiven Druck auf ein Volumenelement. Wie Abbildung 2.4 zeigt, tragen die Spannungskomponenten $\sigma_{\mathrm{xx}}, \sigma_{\mathrm{yy}}$ und $\sigma_{\mathrm{zz}}$ zum Druck bei:

$$
\sigma_{\mathrm{h}}=-\frac{1}{3}\left(\sigma_{11}+\sigma_{22}+\sigma_{33}\right)
$$

Alle Diagonalelemente des Spannungstensors einer Schraubenversetzung verschwinden nach Gleichung 2.15, so dass eine Schraubenversetzungen keinen hydrostatischen Druck bewirkt.

$$
\sigma_{\mathrm{h}}(\text { Schraubenversetzung })=0
$$

Für eine Stufenversetzung ergibt sich der hydrostatische Druck aus den Gleichungen 2.16.

$$
\sigma_{\mathrm{h}}(\text { Stufenversetzung })=\frac{\mathrm{Gb}(1+\mathrm{v})}{3 \pi(1-\mathrm{v})} \cdot \frac{\mathrm{y}}{\mathrm{x}^{2}+\mathrm{y}^{2}}
$$

In Zylinderkoordinaten (vgl. Abb. 2.5) lautet das Ergebnis folgendermaßen:

$$
\begin{aligned}
\sigma_{\mathrm{h}}(\text { Stufenversetzung }) & =-\frac{\mathrm{Gb}(1+v)}{3 \pi(1-v)} \cdot \frac{\sin \vartheta}{\mathrm{r}} \\
& =-\frac{\mathrm{Eb}}{6 \pi(1-v)} \cdot \frac{\sin \vartheta}{\mathrm{r}}
\end{aligned}
$$

Die zweite Identität ergibt sich unmittelbar aus Gleichung 2.12. 

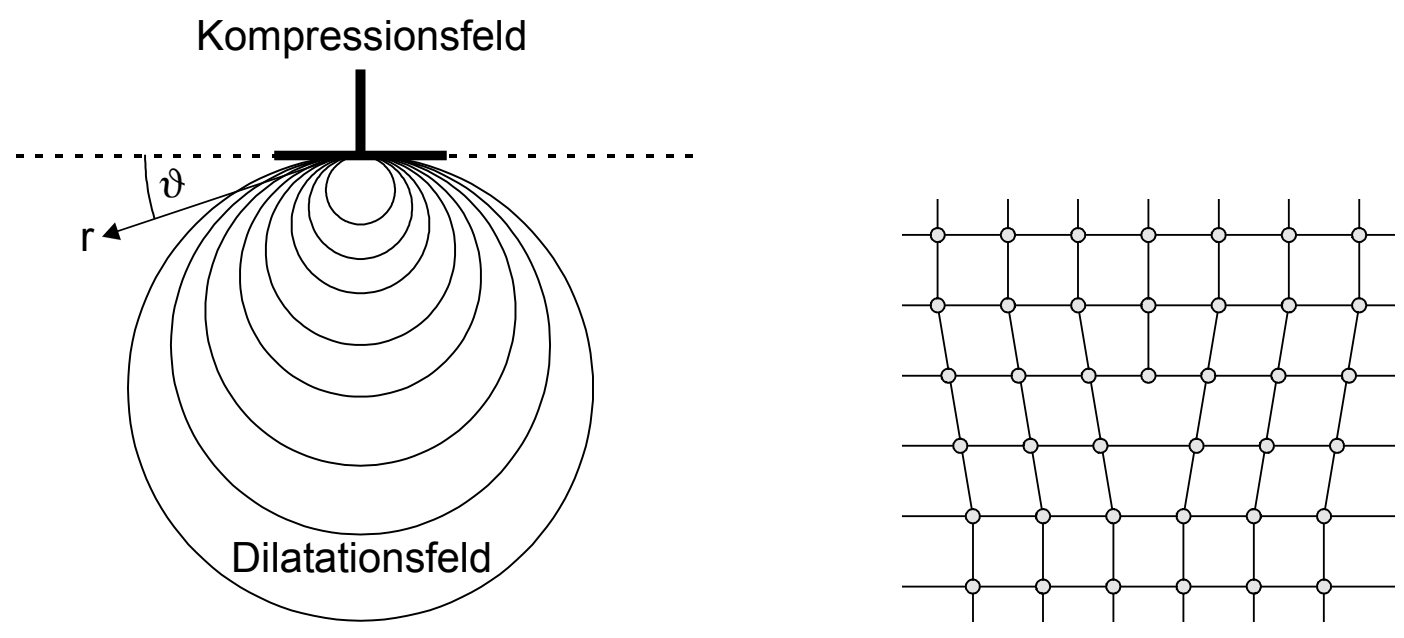

Abbildung 2.5: links: Stufenversetzung mit Linien konstantem hydrostatischen Drucks (Kreise) rechts: Schema einer Stufenversetzung

Der durch eine Stufenversetzung, bzw. den Stufenanteil einer gemischten Versetzung, verursachte hydrostatische Druck fällt wie $1 / \mathrm{r}$ ab, wobei $\mathrm{r}$ der Abstand von der Versetzungslinie in der Ebene senkrecht zum Linienelement ist (vgl. Abb. 2.5). Abbildung 2.5 zeigt weiterhin Linien mit konstantem hydrostatischen Druck als Kreise, die das Linienelement ds (senkrecht zur Zeichenebene) berühren. Der jeweiligen Mantelfläche eines entsprechend orientierten Zylinders eines ungekrümmten Bereichs der Versetzungslinie wird folglich ein hydrostatischer Druck

$$
\sigma_{\mathrm{h}}(\mathrm{R})=-\frac{\mathrm{Eb}}{6 \pi(1-\mathrm{v})} \cdot \frac{1}{2 \mathrm{R}}
$$

zugeordnet, wobei R der Radius des Zylinders ist. Die eingeschobene Halbebene bewirkt eine Kompression des Gitters oberhalb der Versetzungslinie $(\vartheta \in[\pi, 2 \pi])$ und eine Dilatation unterhalb $(\vartheta \in[0, \pi])$. Eine Gitteraufweitung $\bar{V}$ (beispielsweise das partielle Atomvolumen von Wasserstoff in Palladium) führt zu einer Änderung $\sigma_{\mathrm{h}} \overline{\mathrm{V}}$ der freien Enthalpie, die dadurch im Dilatationsfeld abgesenkt wird. Daraus folgt eine attraktive Wechselwirkung von Zwischengitteratomen und dem Dilatationsfeld einer Versetzung, die rein elatisch begründet ist. Mit diesem Ergebnis wird in Kapitel 2.4 die Segregation von Wasserstoff und Deuterium erklärt. 


\subsection{Modell zur Segregation von Wasserstoff an Versetzungen}

Aufgrund der großen Beweglichkeit des Wasserstoffs, auch bei Raumtemperatur, befinden sich die interstitiell gelösten Wasserstoffatome ${ }^{2}$ im ungestörten Gitter mit den Wasserstoffatomen im Spannungsfeld einer Versetzung und der Gasphase im thermodynamischen Gleichgewicht. Dieses liegt genau dann vor, wenn die chemischen Potentiale $\mu_{\alpha}$ des Wasserstoffs in allen Gebieten $\alpha$ identisch sind:

$$
\mu=\mu_{\mathrm{f}}=\mu_{\mathrm{s}}
$$

Dabei bezeichnet, wie bereits zuvor, $\mu_{\mathrm{f}}$ das chemische Potential des „freien” Wasserstoffs im ungestörten Gitter, $\mu_{\mathrm{s}}$ bezieht sich auf das Spannungsfeld und $\mu$ auf die Gasphase. Die Terme für das chemische Potential werden im Folgenden für die drei oben genannten Bereiche entwickelt. Da die Spannungen um eine Versetzung nahe dem Kern nicht elastizitätstheoretisch behandelt werden können, wird zusätzlich zu $\mu_{\mathrm{s}}$ das chemische Potential $\mu_{\mathrm{ss}}$ im Segregationsgebiet diskutiert.

Mit $\mathrm{c}_{\mathrm{f}} \ll 1$ ergibt sich das chemische Potential $\mu_{\mathrm{f}}$ eines Wasserstoffatoms im ungestörten Gitter aus Gleichung 2.4:

$$
\mu_{\mathrm{f}}=\mu_{\mathrm{H}}^{0}+\mathrm{kT} \cdot \ln \left(\mathrm{c}_{\mathrm{f}}\right)
$$

Der Beitrag $\Delta \mu_{\mathrm{h}}$ eines isotropen Spannungsfeldes zum chemischen Potential eines Zwischengitteratoms wird durch den hydrostatischen Druck beschrieben [Li66]

$$
\Delta \mu_{\mathrm{h}}=\sigma_{\mathrm{h}} \cdot \overline{\mathrm{V}}_{\mathrm{H}}
$$

und entspricht der Änderung des chemischen Potentials eines Zwischengitteratoms im elastischen Feld einer Versetzung im Vergleich zum ungestörten Gitter. Da die Gitteraufweitung im Palladium sowohl für ein Wasserstoffatom als auch für ein Deuteriumatom 2,9 $\AA^{3}$ beträgt [Pei78], ist die Wechselwirkung isotopenunabhängig. Mit Gleichung 2.21 erhält man die Wechselwirkung als Funktion des Zylinderradius R:

$$
\Delta \mu_{\mathrm{h}}(\mathrm{R})=-\frac{\mathrm{Eb} \overline{\mathrm{V}}_{\mathrm{H}}}{12 \pi(1-\mathrm{v})} \cdot \frac{1}{\mathrm{R}}
$$

\footnotetext{
${ }^{2}$ Die Betrachtungen in diesem Kapitel werden analog für Wasserstoff und Deuterium angestellt. Zur sprachlichen Vereinfachung wird stellvertretend für beide Isotope von Wasserstoff gesprochen, wenn nicht bei Isotopeneffekten unterschieden werden muss.
} 
Damit kann man das chemische Potential $\mu_{\mathrm{s}}$ eines Wasserstoffatoms im Dilatationsfeld einer Stufenversetzung ausdrücken durch:

$$
\mu_{\mathrm{s}}=\mu_{\mathrm{H}}^{0}+\mathrm{kT} \cdot \ln \left(\mathrm{c}_{\mathrm{s}}(\mathrm{R})\right)+\Delta \mu_{\mathrm{h}}(\mathrm{R})
$$

Die räumliche Variation der Wasserstoffkonzentration besitzt somit dieselbe Zylindergeometrie, die Gleichung 2.21 zugrunde liegt.

Nahe dem Versetzungskern kann die Wasserstoffkonzentration so groß werden, dass zusätzlich noch ein $\mathrm{HH}-$ Wechselwirkungsterm $\Delta \mu_{\mathrm{HH}}$ berücksichtigt werden muss. Außerdem versagt dort obige kontinuumsmechanische Behandlung der elastischen Wechselwirkung mit dem hydrostatischen Druck als Funktion des Radius R. Atomistische Rechnungen ermöglichen jedoch die Beschreibung der Wasserstoff-Defekt-Wechselwirkung im Versetzungskern. Neben der effective-medium Theorie [Nør80, Nor86] wurden EAM-Potentiale (Embedded Atom Method) [Bas98] erfolgreich angewandt. Letztere Rechnungen zeigen bei Nickel eine so starke Bindung von Wasserstoff nahe dem Versetzungskern, dass sich ein stoichiometrisches Hydrid bildet. Die mögliche Annahme, der Beitrag $\Delta \mu_{\mathrm{HH}}$ entspräche der Hydridbildungsenthalpie, widerspricht jedoch inelastischen Neutronenstreumessungen der lokalen Moden von Wasserstoff in verformtem und unverformten Palladium [Kir88]. In beiden Fällen liegt das Peakmaximum bei $68 \mathrm{meV}$, wie man es für Wasserstoff in der $\alpha$-Phase erwartet [Rus84]. In der $\beta$-Phase gebundener Wasserstoff besitzt einen Peak bei $59 \mathrm{meV}$ [Rus85]. Wäre der Wasserstoff in den verformten Proben derartig gebunden, so hätte man eine erhöhte Intensität bei dieser Energie auch für eine der $\alpha$-Phase entsprechende Wasserstoffkonzentration finden müssen. Es zeigte sich jedoch lediglich eine Peakverbreiterung, die als Folge der Platzenergieverteilung um eine Stufenversetzung zu verstehen ist. Dennoch liefern gasvolumetrische Messungen [Fla76a] oder Messungen der EMK [Kir81b] übereinstimmend Werte von ca. $-18 \mathrm{KJ} / \mathrm{mol}$ für $\Delta \mu_{\mathrm{HH}}$, die als chemische Wechselwirkung interpretiert werden. Eine rein elastische Wechselwirkung ist in [Fi193] modelliert und führt zu einer Segregation mit scharfer Grenzfläche an den Versetzungen. Es ist also davon auszugehen, dass sich nahe dem Versetzungskern ein Bereich mit hoher Wasserstoffkonzentration bildet, ohne dass dieser der $\beta$-Phase entspricht. Den zu erwartenden Konzentrationsverlauf zeigt Abbildung 2.6. 


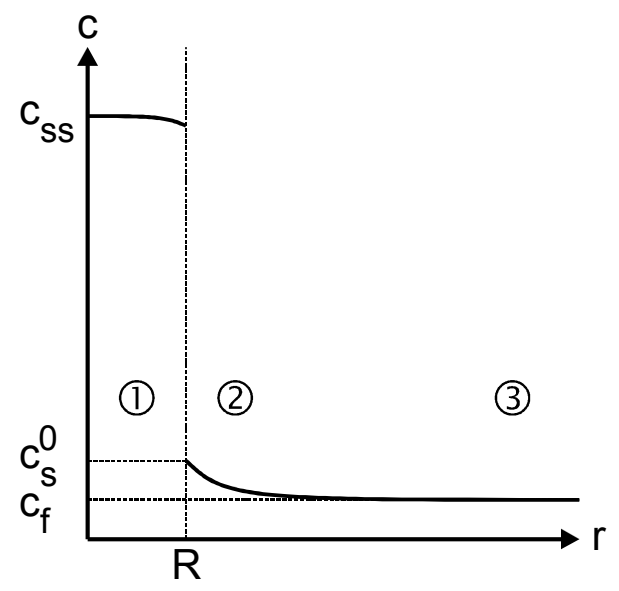

Abbildung 2.6: Der H-Konzentrationsverlauf im Dilatationsfeld einer Stufenversetzung im Ursprung. (1) für $\mathrm{r}<\mathrm{R}$ ist eine $\mathrm{HH}-W e c h s e l w i r k u n g$ berücksichtigt. (2) der Konzentrationsverlauf für $\mathrm{r}>\mathrm{R}$ ergibt sich aufgrund der elastischen Wechselwirkung und nähert sich der Konzentration $\mathrm{c}_{\mathrm{f}}$ des idealen Gitters (3).

Im zylinderförmigen Segregationsgebiet wird das chemische Potential $\mu_{\mathrm{ss}}$ eines Wasserstoffatoms ausreichend durch Annahme einer konstanten Konzentration $\mathrm{c}_{\mathrm{ss}}$ und eines effektiven Beitrags $\Delta \mu_{\mathrm{ss}}$ für die elastische und chemische Wechselwirkung $\left(\Delta \mu_{\mathrm{h}}, \Delta \mu_{\mathrm{HH}}\right)$ beschrieben:

$$
\mu_{\mathrm{ss}}=\mu_{\mathrm{H}}^{0}+\mathrm{kT} \cdot \ln \left(\frac{\mathrm{c}_{\mathrm{ss}}}{1-\mathrm{c}_{\mathrm{ss}}}\right)+\Delta \mu_{\mathrm{ss}}
$$

Im Kompressionsfeld der Stufenversetzung ist die Wasserstoffkonzentration abgesenkt. Dieser Effekt ist ausgehend von kleinen Konzentrationen $\mathrm{c}_{\mathrm{f}}$ im Vergleich zur Segregation im Dilatationsfeld klein und wird im Folgenden nicht weiter berücksichtigt.

Wie zuvor erwähnt, gibt es neben der Gasphase drei Bereiche der interstitiellen Lösung, die sich miteinander im Gleichgewicht befinden müssen:

$$
\mu_{\mathrm{f}}=\mu_{\mathrm{s}}=\mu_{\mathrm{ss}}
$$

Abbildung 2.6 zeigt die erwarteten Konzentrationsverläufe im Dilatationsfeld. Bereich 1 ist das oben beschriebene zylinderförmige Segregationsgebiet mit scharfer Grenzfläche. Die Wasserstoffkonzentration $\mathrm{c}_{\mathrm{ss}}$ entspricht der $\beta$-Phase. Im Bereich 2 ist die H-Konzentration bedingt durch die Wechselwirkung mit dem elastischen Feld der Stufenversetzung höher als die Konzentration $\mathrm{c}_{\mathrm{f}}$ des ungestörten Gitters (Bereich 3). 
Zentrale Aufgabe dieser Arbeit ist die Untersuchung des zylinderförmigen Segregationsgebietes. Dazu wurde ein Modell entwickelt, mit welchem der Radius R allein aufgrund des Gleichgewichts von Bereich 2 und 3 zu bestimmen ist. Zunächst setzt man unter der Bedingung eines identischen Standardpotentials $\mu_{\mathrm{H}}^{0}$ die Gleichungen 2.23 und 2.26 gleich:

$$
\mathrm{kT} \cdot \ln \left(\mathrm{c}_{\mathrm{f}}\right)=\mathrm{kT} \cdot \ln \left(\mathrm{c}_{\mathrm{s}}^{0}\right)-\frac{\mathrm{Eb} \overline{\mathrm{V}}_{\mathrm{H}}}{12 \pi(1-\mathrm{v})} \cdot \frac{1}{\mathrm{R}}
$$

Dabei bezeichnet $\mathrm{c}_{\mathrm{s}}^{0}$ die maximale Löslichkeit der $\alpha$-Phase, die entsprechend Abbildung 2.6 am Rand des Segregationsgebietes vorliegt. Alle in Gleichung 2.29 eingehenden Größen sind experimentell zugänglich und in Tabelle 2.2 zusammengefasst.

Tabelle 2.2: $\quad$ Materialparameter für Palladium.

\begin{tabular}{|c|c|c|}
\hline Elastititätsmodul & $\mathrm{E}$ & $18,08 \cdot 10^{10} \mathrm{~Pa}$ \\
\hline Poissonsche Querkontraktionszahl & $\mathrm{v}$ & 0,39 \\
\hline Burgersvektor & $\mathrm{b}=\frac{\mathrm{a}_{\mathrm{Pd}}}{\sqrt{2}}=\frac{3,89 \cdot 10^{-10} \mathrm{~m}}{\sqrt{2}}$ & $2,751 \cdot 10^{-10} \mathrm{~m}$ \\
\hline partielles Atomvolumen & $\mathrm{V}_{\mathrm{H}}=\mathrm{V}_{\mathrm{D}}$ & $2,9 \cdot 10^{-30} \mathrm{~m}^{3}$ \\
\hline $\begin{array}{c}\text { maximale Löslichkeit der } \alpha \text {-Phase } \\
\text { in versetzungsreichem Pd }\end{array}$ & $\mathrm{c}_{\mathrm{s}}^{0}$ & $0,008 \mathrm{H} / \mathrm{Pd}$ \\
\hline Besetzung im Hydrid & $\mathrm{c}_{\mathrm{ss}}$ & $0,6 \mathrm{H} / \mathrm{Pd}$ \\
\hline
\end{tabular}

Aus Gleichung 2.29 folgt ein Zusammenhang zwischen dem Radius R des Segregationsgebietes und der Wasserstoffkonzentration $\mathrm{c}_{\mathrm{f}}$ im ungestörten Gitter:

$$
c_{f}=c_{s}^{0} \cdot \exp \left(-\frac{A}{R}\right) \quad A=\frac{E b \bar{V}_{H}}{12 \pi(1-v) k T}
$$

Mit den in Tabelle 2.2 aufgeführten Parametern ergibt sich bei Raumtemperatur $(\mathrm{T}=293 \mathrm{~K})$ für die Konstante A der Wert $\mathrm{A}=15,51 \cdot 10^{-10} \mathrm{~m}$. 
Als weitere Bedingung muss Gleichung 2.3 erfüllt sein: $\mathrm{c}=\mathrm{c}_{\mathrm{f}}+\Delta \mathrm{c}$. Dabei wird der Beitrag des Spannungsfeldes außerhalb des Segregationsgebietes $(r>R)$ vernachlässigt. Der Volumenbruchteil des Segregationsgebietes ergibt sich aus der Versetzungsdichte $\zeta \mathrm{zu} \zeta \cdot \pi \mathrm{R}^{2}$. Innerhalb dieses Volumens wird eine konstante Konzentration $\mathrm{c}_{\mathrm{ss}}$ angenommen, so dass sich der folgende Zuammenhang ergibt:

$$
\mathrm{c}=\mathrm{c}_{\mathrm{ss}} \cdot \zeta \pi \mathrm{R}^{2}+\mathrm{c}_{\mathrm{s}}^{0} \cdot \exp \left(-\frac{\mathrm{A}}{\mathrm{R}}\right)
$$

In dieser Arbeit wird die radiale Ausdehnung $\mathrm{R}$ des Segregationsgebietes durch Vergleich der Kleinwinkelneutronenstreukurven mit dem Zylindermodell (Kapitel 3.3) bestimmt. Das hier entwickelte Modell verknüpft diesen Zylinderradius mit experimetell bestimmbaren Größen. Durch Anpassung von Gleichung 2.31 an die Zylinderradien (Kapitel 5.2.1) können diese überprüft werden. 


\section{Untersuchung des Segregationsgebietes mit Neutronenstreuung}

Zur Untersuchung der räumlichen Verteilung von Wasserstoff und Deuterium in versetzungsreichem Palladium wird in der vorliegenden Arbeit die Methode der Kleinwinkelneutronenstreuung (SANS) verwendet. Sie stellt derzeit die einzige Möglichkeit dar, den experimentellen Nachweis für das zylinderförmige Segregationsgebiet zu erbringen, welcher in Kapitel 2 gefordert wurde. In diesem Kapitel werden Modellrechnungen des Streuverhaltens der erwarteten Streuobjekte entwickelt, mit denen in Kapitel 5.2 die experimentell ermittelten Streukurven interpretiert werden.

\subsection{Grundlagen der Neutronenstreuung}

Die Bedeutung der Neutronenstreuung bei der Untersuchung von Festkörpern basiert auf den grundlegenden Eigenschaften des Neutrons, welche seine Wechselwirkung mit Materie bestimmen.

$\begin{array}{ll}\text { Ladung } & \mathrm{Z}_{\mathrm{n}}=0 \\ \text { Masse } & \mathrm{m}_{\mathrm{n}}=1,675 \cdot 10^{-27} \mathrm{~kg} \\ \text { Spin } & \mathrm{S}_{\mathrm{n}}=1 / 2 \hbar \\ \text { elektrisches Dipolmoment } & \mathrm{P}_{\mathrm{n}}=0 \\ \text { magnetisches Dipolmoment } & \mu_{\mathrm{n}}=-1,913 \cdot \mu_{\mathrm{Kern}} \\ \text { Halbwertszeit } & \tau_{1 / 2}=888 \mathrm{~s}\end{array}$

Aufgrund der fehlenden Ladung werden Neutronen nicht an der Elektronenhülle eines Atoms sondern am Atomkern gestreut. Ihre Wechselwirkung mit Materie ist im Vergleich zu Röntgenquanten schwächer. Daher ist es möglich, auch massive Proben mit Neutronen zu durchstrahlen.

Trifft eine ebene Welle auf ein punktförmiges Streuzentrum, geht von diesem nach dem Huygensschen Prinzip eine kugelsymmetrische Streuwelle aus.

einfallende ebene Welle $\quad \mathrm{e}^{\mathrm{i} \overrightarrow{\mathrm{k}}_{0} \cdot \overrightarrow{\mathrm{r}}}$

gestreute Kugelwelle $\quad-\frac{b}{|\vec{r}|} e^{i\left|\vec{k}_{1}\right| \cdot|\vec{r}|}$ 
Dabei bezeichnet $\overrightarrow{\mathrm{k}}_{0}$ den Wellenzahlvektor der einfallenden Neutronenwelle und $\overrightarrow{\mathrm{k}}_{1}$ den der gestreuten Welle. Gemäß der Impuls- und Energieerhaltung ergibt sich bei einem Streuexperiment ein Impuls- und Energieübertrag:

$$
\begin{aligned}
& \hbar \overrightarrow{\mathrm{Q}}=\hbar \overrightarrow{\mathrm{k}}_{0}-\hbar \overrightarrow{\mathrm{k}}_{1} \\
& \hbar \omega=\frac{\hbar^{2} \overrightarrow{\mathrm{k}}_{0}^{2}}{2 \mathrm{~m}_{\mathrm{n}}}-\frac{\hbar^{2} \overrightarrow{\mathrm{k}}_{1}^{2}}{2 \mathrm{~m}_{\mathrm{n}}}
\end{aligned}
$$

Der Streuvektor $\overrightarrow{\mathrm{Q}}=\overrightarrow{\mathrm{k}}_{0}-\overrightarrow{\mathrm{k}}_{1}$ wird durch den Impulsübertrag definiert. Findet ein Energieaustausch der Neutronenwelle mit dem streuenden System statt $(\omega \neq 0)$, so spricht man von inelastischer Streuung. Gilt $\left|\overrightarrow{\mathrm{k}}_{0}\right|=\left|\overrightarrow{\mathrm{k}}_{1}\right|$ und damit $\omega=0$, so liegt der elastische Fall vor.

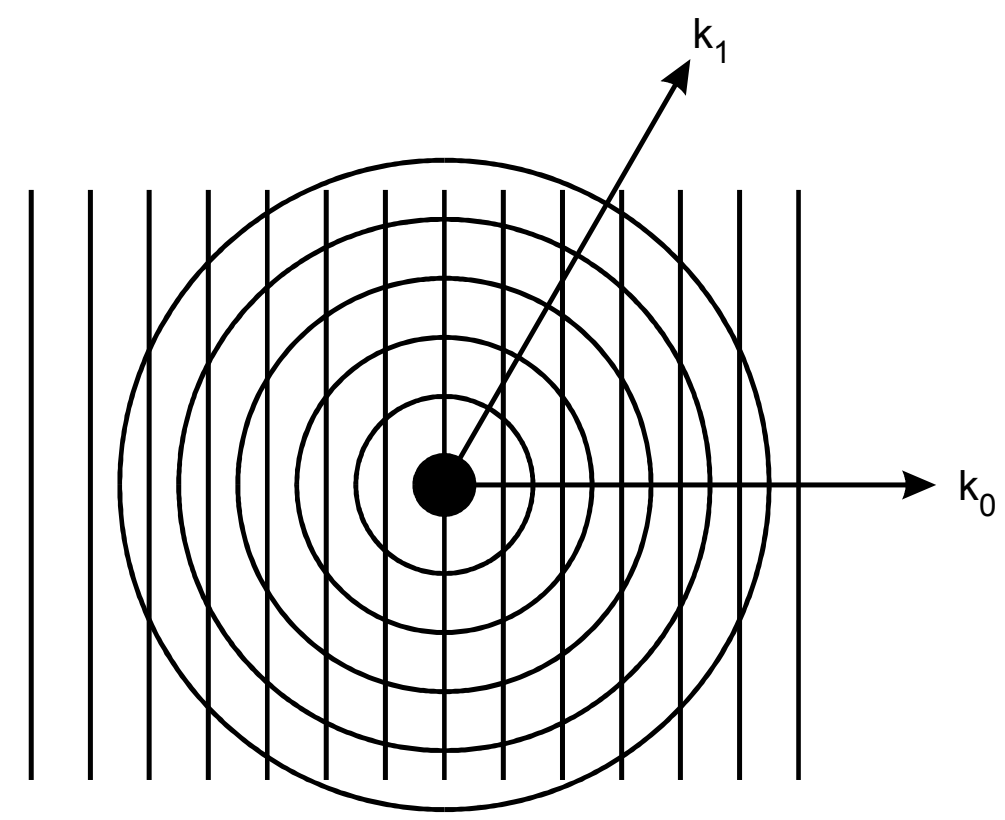

Abbildung 3.1: Schematisch Skizze eines Streuexperimentes. Die einfallende ebene Welle mit dem Wellenzahlvektor $\mathrm{k}_{0}$ trifft auf ein Streuzentrum, von dem eine Kugelwelle ausgeht.

Die Intensität wird bei Streuexperimenten als Streuquerschnitt angegeben. Unter dem totalen Streuquerschnitt $\sigma$ versteht man den Anteil der gestreuten Neutronen:

$$
\sigma=\frac{\text { Anzahl gestreuter Neutronen pro Zeiteinheit }}{\text { Fluß einfallender Neutronen }}
$$

Der differentielle Streuquerschnitt d $\sigma$ pro Raumwinkelelement $\mathrm{d} \Omega$ wird als makroskopischer Streuquerschnitt $\mathrm{d} \Sigma / \mathrm{d} \Omega$ bezeichnet:

$$
\frac{\mathrm{d} \Sigma}{\mathrm{d} \Omega}=\frac{\text { Anzahl der nach } \mathrm{d} \Omega \text { gestreuten Neutronen pro Zeiteinheit }}{\text { Flu } ß \text { einfallender Neutronen } \cdot \mathrm{d} \Omega}
$$


Eine Konsequenz aus der Streuung am Atomkern ist, dass Isotope unterschiedlich stark streuen. Analog zum Strukturfaktor bei der Röntgenstreuung wird die Amplitude der Streuwelle als das Streuvermögen eines Atoms definiert. Man bezeichnet diese Größe als die Streulänge b, die vom Kernradius, der Kernstruktur, dem Kernspin und der Energie der Neutronen abhängt.

Bei der Streuung an einem Kristall aus chemisch identischen Atomen sind zwei Anteile zu unterscheiden. Die kohärente Streuung wird beschrieben durch eine mittlere Streulänge aller chemisch identischen Atome:

$$
\mathrm{b}_{\text {kohärent }}=\langle\mathrm{b}\rangle
$$

Die kohärent gestreuten Neutronen sind interferenzfähig und damit abhängig vom Streuvektor. Dies gilt im elastischen Fall nicht für die inkohärente Streuung, die durch die Fluktuationen der Streulängen beschrieben wird.

$$
\mathrm{b}_{\text {inkohärent }}=\sqrt{\left\langle\mathrm{b}^{2}\right\rangle-\langle\mathrm{b}\rangle^{2}}
$$

Für diese Arbeit sind die Streulängen von Wasserstoff H, Deuterium D und Palladium Pd von Bedeutung. Sie ergeben sich aus den Streulängen der einzelnen Kernzustände und deren Wahrscheinlichkeit P.

$\begin{array}{llll}\mathrm{H} \quad \mathrm{H}^{1} \quad \mathrm{~J}=1 \text { (parallele Spins) } & \mathrm{b}^{+}=10,8 \mathrm{fm} & \mathrm{P}\left(\mathrm{b}^{+}\right)=\frac{3}{4} \\ & \mathrm{~J}=0 \text { (antiparallele Spins) }) & \mathrm{b}^{-}=-47,4 \mathrm{fm} & \mathrm{P}\left(\mathrm{b}^{-}\right)=\frac{1}{4} \\ & \mathrm{~b}_{\text {kohärent }}=-3,739 \mathrm{fm} \\ & \mathrm{b}_{\text {inkohärent }}=25,27 \mathrm{fm}\end{array}$


Den makroskopischen Streuquerschnitt erhält man aus der Interferenz von N Kugelwellen:

$$
\frac{\mathrm{d} \Sigma}{\mathrm{d} \Omega}=\mathrm{N} \cdot\left|\iiint_{\text {Teilchen }} \Delta \rho \cdot \mathrm{e}^{\mathrm{i} \overrightarrow{\mathrm{Q}} \cdot \overline{\mathrm{r}}} \mathrm{d}^{3} \overrightarrow{\mathrm{r}}\right|^{2}+\sigma_{\text {inkohärent }}
$$

Dabei bezeichnet $\Delta \rho=\left\langle b_{\text {kohärent }}\right\rangle$ die Streulängendichtedifferenz des Teilchens zur Matrix und $\sigma_{\text {inkohärent }}=4 \pi \cdot b_{\text {inkohärent }}^{2}$ den inkohärenten Streuquerschnitt.

\subsection{Typische Streukurven ausgewählter Streuobjekte}

Die makroskopischen Streuquerschnitte einfacher Objekte besitzen typische Streukurven. Allgemeine Darstellungen werden von Kostorz [Kos93] und Glatter und Kratky [Gla82] gegeben. Im Folgenden werden das Guinierverhalten und das Porodverhalten vorgestellt.

\subsubsection{Guinierverhalten}

Die Streuung von Kugeln mit einer Streulängendichtedifferenz $\Delta \rho$ zur homogenen Matrix und einer Volumendichte N kann entsprechend Gleichung 3.8 berechnet werden:

$$
\frac{\mathrm{d} \Sigma}{\mathrm{d} \Omega}=\mathrm{N} \cdot\left(\frac{4 \pi \cdot \Delta \rho}{\mathrm{Q}}\right)^{2} \cdot\left|\frac{\sin \mathrm{QR}}{\mathrm{Q}^{2}}-\frac{\mathrm{R} \cdot \cos \mathrm{QR}}{\mathrm{Q}}\right|^{2}
$$

Für QR $\rightarrow 0$ entspricht das Betragsquadrat in der Guinier-Näherung [Gui63] einer Exponentialfunktion. Unter Verwendung des Volumens V eines Teilchens erhält man folgenden Zusammenhang:

$$
\frac{\mathrm{d} \Sigma}{\mathrm{d} \Omega}=\mathrm{N} \cdot \mathrm{V}^{2} \cdot \Delta \rho^{2} \cdot \exp \left(-\frac{\mathrm{Q}^{2} \mathrm{R}^{2}}{5}\right)
$$

Die Guinier-Näherung ist für eine gaußförmige Streulängendichte exakt:

$$
\frac{\mathrm{d} \Sigma}{\mathrm{d} \Omega}=\mathrm{N} \cdot \mathrm{V}^{2} \cdot \Delta \rho^{2} \cdot \exp \left(-\frac{\mathrm{Q}^{2}\left\langle\mathrm{R}^{2}\right\rangle}{3}\right)
$$

Dabei bezeichnet $\left\langle\mathrm{R}^{2}\right\rangle$ den Gyrationsradius des Teilchens. Die Guinier-Gleichung 3.11 gilt für eine beliebige Teilchenform, wenn QR klein ist. 


\subsubsection{Porodverhalten}

Betrachtet man Gleichung 3.9 für den Fall QR $\gg 1$, so kann man das Betragsquadrat in der folgenden Gleichung durch sein Maximum von eins annähern [Por51, Por52].

$$
\frac{\mathrm{d} \Sigma}{\mathrm{d} \Omega}=\mathrm{N} \cdot\left(\frac{4 \pi \cdot \Delta \rho}{\mathrm{Q}}\right)^{2} \cdot \frac{\mathrm{R}^{2}}{\mathrm{Q}^{2}} \cdot\left|\frac{\sin \mathrm{QR}}{\mathrm{QR}}-\cos \mathrm{QR}\right|^{2}
$$

Fasst man zusätzlich $\mathrm{N} \cdot 4 \pi \mathrm{R}^{2}$ zur Gesamtoberfläche $\mathrm{S}$ pro Einheitsvolumen zusammen, so erhält man das Porod-Gesetz:

$$
\frac{\mathrm{d} \Sigma}{\mathrm{d} \Omega}=\frac{4 \pi \cdot \mathrm{S} \cdot(\Delta \rho)^{2}}{\mathrm{Q}^{4}}
$$

Das Porod-Gesetz gilt allgemein für Teilchen mit scharfen Grenzflächen und homogener Dichte. Insbesondere muss Q größer als der Reziprokwert der kürzesten Teilchendimension sein, damit $\mathrm{QR} \gg 1$ erfüllt ist. Umgekehrt bedeutet dies, dass die Teilchendimension mindestens dem Reziprokwert des kleinsten Streuvektors entspricht, bei dem das Porodverhalten beobachtet wurde.

\subsection{Das Streuverhalten des Segregationszylinders}

Zentrale Aufgabe der vorliegenden Arbeit ist die Untersuchung des zylinderförmigen Segregationsgebietes mit der Methode der Kleinwinkelneutronenstreuung. Daher wird das Streuverhalten eines Zylinders in Anlehnung an Atkinson und Hirsch [Atk58] hergeleitet.

Aus der Änderung $\Delta \rho$ der Streulängendichte ist der makroskopische Streuquerschnitt zu berechnen:

$$
\frac{\mathrm{d} \Sigma}{\mathrm{d} \Omega}=\mathrm{N}_{\mathrm{d}}\left\langle\left|\iint_{V_{\mathrm{V} o l}} \Delta \rho(\mathrm{r}, \vartheta) \mathrm{e}^{\mathrm{i} \overrightarrow{\mathrm{Q}} \overrightarrow{\mathrm{x}}} \mathrm{d}^{3} \overrightarrow{\mathrm{x}}\right|^{2}\right\rangle
$$

Als streuendes Objekt, in dem $\Delta \rho \neq 0$ gilt, werden entsprechend Kapitel 2 Zylinder angenommen. Die Auswertung von Gleichung 3.14 erfolgt in drei Schritten:

1. Das Volumenintegral über den streuenden Bereich wird berechnet. Dabei muß der Integrand geeignet entwickelt werden. Als Ergebnis erhält man die Amplitude eines Zylinders mit einer konstanten Streulängendichtedifferenz $\Delta \rho$.

2. Die Intensität eines Zylinders wird als Betragsquadrat der Amplitude bestimmt. Dabei werden Terme höherer Ordnung nicht berücksichtigt, weil sie einen vernachlässigbaren Beitrag zum Ergebnis liefern. 
3. Die Intensität eines Zylinders wird räumlich gemittelt. Damit bekommt man die Intensität eines Ensembles identischer streuender Objekte, die in alle Raumrichtungen gleichverteilt sind.

Zuerst sollen einfache mathematische Vorbereitungen zusammengefasst werden.

Aufgrund der Zylindersymmetrie des Problems werden der Ortsvektor $\overrightarrow{\mathrm{x}}$ und der Streuvektor $\overrightarrow{\mathrm{Q}}$ in Zylinderkoordinaten (vgl. Abb. 3.2) dargestellt:

$$
\vec{x}=\left(\begin{array}{c}
r \cos \vartheta \\
r \sin \vartheta \\
z
\end{array}\right) \wedge \vec{Q}=Q\left(\begin{array}{c}
\sin \chi \cos \xi \\
\sin \chi \sin \xi \\
\cos \chi
\end{array}\right)
$$

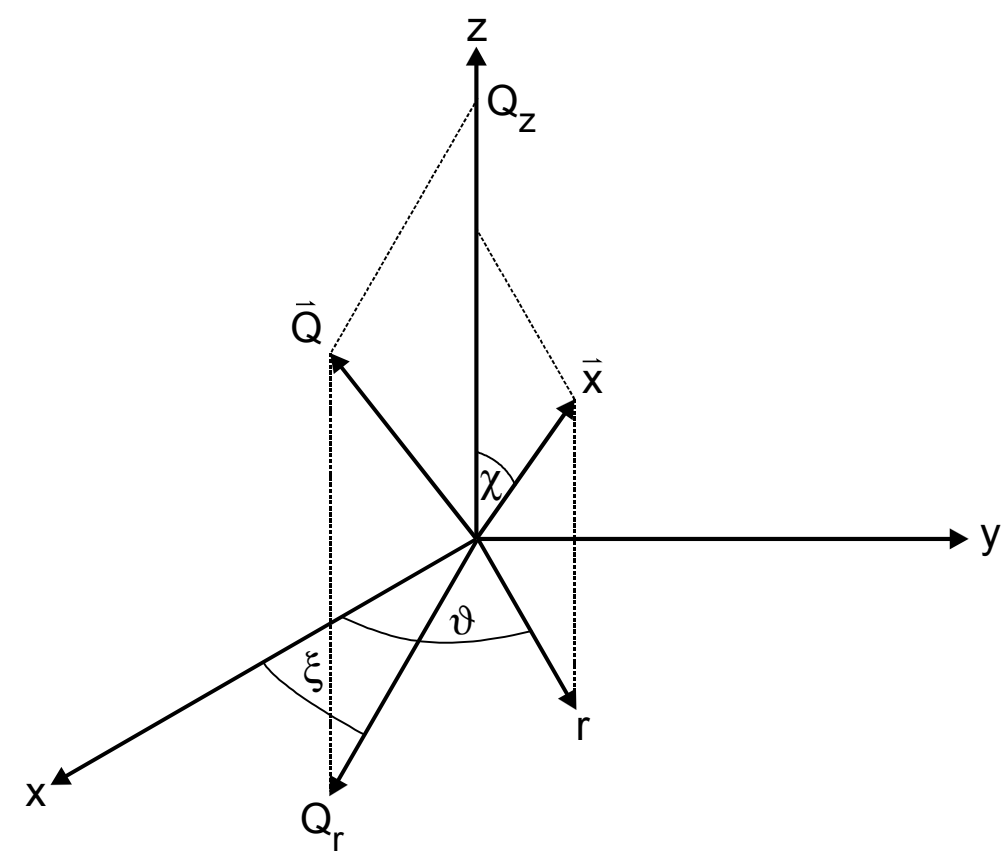

Abbildung 3.2: Zylinderkoordinaten

Damit ergibt sich für das Skalarprodukt $\overrightarrow{\mathrm{Q}} \cdot \overrightarrow{\mathrm{x}}$ in Zylinderkoordinaten unter Verwendung des Additionstheorems (3.17):

$$
\begin{aligned}
& \overrightarrow{\mathrm{Q}} \cdot \overrightarrow{\mathrm{x}}=\mathrm{Qr}(\sin \chi \cos \xi \cos \vartheta+\sin \chi \sin \xi \sin \vartheta)+\mathrm{Q} \cos (\chi) \mathrm{z} \\
& =\mathrm{rQ} \sin \chi \cos (\xi-\vartheta)+\mathrm{Q} \cos (\chi) \mathrm{z} \\
& \cos (\alpha \pm \beta)=\cos \alpha \cos \beta \mp \sin \alpha \sin \beta
\end{aligned}
$$


Das Integral stellt im mathematischen Sinne eine Fouriertransformation dar und führt auf eine Besselfunktion. Die analytische Lösung kann approximiert werden, wenn man den Integranden entwickelt. Dafür werden die ersten drei Glieder der Exponentialreihe verwendet:

$$
\exp (\mathrm{x})=\sum_{\mathrm{k}=0}^{\infty} \frac{\mathrm{x}^{\mathrm{k}}}{\mathrm{k} !} \approx 1+\mathrm{x}+\frac{1}{2} \mathrm{x}^{2}+\mathrm{O}\left(\mathrm{x}^{3}\right)
$$

In dieser Näherung lautet der Exponentialterm aus Gleichung 3.14 folgendermaßen:

$$
\exp (\mathrm{irQ} \sin \chi \cos (\xi-\vartheta)) \approx 1+\mathrm{irQ} \sin \chi \cos (\xi-\vartheta)-\frac{1}{2} \mathrm{r}^{2} \mathrm{Q}^{2} \sin ^{2} \chi \cos ^{2}(\xi-\vartheta)
$$

Damit kann man das Volumenintegral auswerten. Dies erfolgt für einen Zylinder der Länge $2 \cdot \mathrm{H}$ und dem Radius $\mathrm{R}$ unter der Annahme eines konstanten Kontrastes $\Delta \rho$ :

$$
\Delta \rho(r, \vartheta, z)=\left\{\begin{array}{cc}
\frac{\mathrm{b} \cdot \mathrm{c}}{\mathrm{V}_{\mathrm{M}}} & \mathrm{r} \leq \mathrm{R} \wedge|\mathrm{z}| \leq \mathrm{H} \\
0 & \text { sonst }
\end{array}\right.
$$

Außerdem kann man die Integration nach $\mathrm{z}$ von der nach $\vartheta$ und $\mathrm{r}$ separieren.

$$
\begin{aligned}
\iiint_{\text {Zylinder }} \Delta \rho(\mathrm{r}, \vartheta) \mathrm{e}^{\mathrm{i} \overrightarrow{\mathrm{Q}} \overrightarrow{\mathrm{x}}} \mathrm{d}^{3} \overrightarrow{\mathrm{x}} & \approx \\
& \int_{-\mathrm{H}}^{\mathrm{H}} \exp (\mathrm{izQ} \cos \chi) \mathrm{dz} . \\
& \cdot \int_{0}^{\mathrm{R}} \int_{0}^{2 \pi}\left[1+\mathrm{irQ} \sin \chi \cos (\xi-\vartheta)-\frac{1}{2} \mathrm{r}^{2} \mathrm{Q}^{2} \sin ^{2} \chi \cos ^{2}(\xi-\vartheta)\right] \mathrm{d} \vartheta \mathrm{rdr} \mathrm{r}
\end{aligned}
$$

Die beiden Integrale liefern das folgende Ergebnis:

$$
\begin{aligned}
& \int_{-H}^{H} \exp (i Q z) d z=\left[\frac{\exp (i z Q \cos \chi)}{i Q \cos \chi}\right]_{-H}^{H}=\frac{2 \sin (H Q \cos \chi)}{Q \cos \chi} \\
& \int_{0}^{\mathrm{R}} \int_{0}^{2 \pi}\left[1+\operatorname{irQ} \sin \chi \cos (\xi-\vartheta)-\frac{1}{2} \mathrm{r}^{2} \mathrm{Q}^{2} \sin ^{2} \chi \cos ^{2}(\xi-\vartheta)\right] \mathrm{d} \vartheta \mathrm{rdr} \\
& =\pi \mathrm{R}^{2}-\frac{\pi}{8} \mathrm{R}^{4} \mathrm{Q}^{2} \sin ^{2} \chi
\end{aligned}
$$


Damit ist der erste Schritt der Lösung von Gleichung 3.14 abgeschlossen und Gleichung 3.24 stellt die Amplitude der Streuung an einem Zylinder dar.

$$
\mathrm{A}(\mathrm{Q}, \chi, \xi)=\frac{2 \mathrm{bc} \pi \mathrm{R}^{2}}{\mathrm{~V}_{\mathrm{M}}} \cdot \frac{\sin (\mathrm{HQ} \cos \chi)}{\mathrm{Q} \cos \chi} \cdot\left(1-\frac{1}{8} \mathrm{R}^{2} \mathrm{Q}^{2} \sin ^{2} \chi\right)
$$

Die Intensität enspricht dem Betragsquadrat der Amplitude. Es werden nur Terme bis $\mathrm{R}^{6}$ berücksichtigt.

$$
\begin{aligned}
|A|^{2} & =\frac{4 b^{2} c^{2} \pi^{2} R^{4}}{V_{M}^{2}} \cdot \frac{\sin ^{2}(H Q \cos \chi)}{Q^{2} \cos ^{2} \chi} \cdot\left(1-\frac{1}{4} R^{2} Q^{2} \sin ^{2} \chi+\frac{1}{64} R^{4} Q^{4} \sin ^{4} \chi\right) \\
& \approx \frac{4 b^{2} c^{2} \pi^{2} R^{4}}{V_{M}^{2}} \cdot \frac{\sin ^{2}(H Q \cos \chi)}{Q^{2} \cos ^{2} \chi} \cdot\left(1-\frac{1}{4} R^{2} Q^{2} \sin ^{2} \chi\right)
\end{aligned}
$$

Gleichung 3.25 stellt die Intensität eines streuenden Zylinders dar, dessen Längsachse den Winkel $\chi$ mit dem Streuvektor Q einschließt. Zur Bestimmung des Streuquerschnittes eines Ensembles von Zylindern mit statistischer Orientierung, ist eine räumliche Mittelung über die Winkel $\xi$ und $\chi$ durchzuführen.

Da die Amplitude $\mathrm{A}(\mathrm{Q}, \chi, \xi)=\mathrm{A}(\mathrm{Q}, \chi)$ nur vom Winkel $\chi$ abhängt, ist dieser geeignet zu substituieren:

$$
\begin{array}{ccc}
\mu=\cos \chi & \Rightarrow & \sin ^{2} \chi=1-\mu^{2} \\
\mathrm{~d} \mu=-\sin \chi \mathrm{d} \chi & \Rightarrow & \mathrm{d} \chi=\frac{-\mathrm{d} \mu}{\sqrt{1-\mu^{2}}}
\end{array}
$$

Entsprechend Gleichung 3.14 ergibt sich der makroskopische Streuquerschnitt zu:

$$
\frac{\mathrm{d} \Sigma}{\mathrm{d} \Omega}=\frac{2 \mathrm{~N}_{\mathrm{d}} \mathrm{b}^{2} \mathrm{c}^{2} \pi^{2} \mathrm{R}^{4}}{\mathrm{Q}^{2} \mathrm{~V}_{\mathrm{M} ;}^{2}} \cdot \int_{-1}^{1} \frac{\sin ^{2}(\mathrm{HQ} \mu)}{\mu^{2}} \cdot\left(1-\frac{1}{4} \mathrm{R}^{2} \mathrm{Q}^{2}\left(1-\mu^{2}\right)\right) \mathrm{d} \mu
$$

Mit $\sin ^{2} x=\frac{1}{2}(1-\cos 2 x)$ und dem Integralsinus $\operatorname{Si}(x)=\int_{0}^{x} \frac{\sin t}{t} d t$ ergeben sich:

$$
\begin{aligned}
\int_{-1}^{1} \frac{\sin ^{2}(\mathrm{HQ} \mu)}{\mu^{2}} \mathrm{~d} \mu & =\int_{-1}^{1} \frac{\mathrm{d} \mu}{2 \mu^{2}}-\int_{-1}^{1} \frac{\cos 2 \mathrm{HQ} \mu}{2 \mu^{2}} \mathrm{~d} \mu \\
& =\left[\frac{-1}{2 \mu}\right]_{-1}^{1}+\left[\frac{\cos 2 \mathrm{HQ} \mu}{2 \mu}\right]_{-1}^{1}+\mathrm{HQ} \int_{-1}^{1} \frac{\sin 2 \mathrm{HQ} \mu}{\mu} \mathrm{d} \mu \\
& =-1+\cos (2 \mathrm{HQ})+2 \mathrm{HQ} \cdot \mathrm{Si}(2 \mathrm{HQ})
\end{aligned}
$$


und:

$$
\begin{aligned}
\int_{-1}^{1} \sin ^{2}(\mathrm{HQ} \mu) & =\left[\frac{\mu}{2}-\frac{\sin (2 \mathrm{HQ} \mu)}{4 \mathrm{HQ}}\right]_{-1}^{1} \\
& =1-\frac{\sin (2 \mathrm{HQ})}{2 \mathrm{HQ}}
\end{aligned}
$$

Zusammengefaßt liefern diese Gleichungen das Ergebnis:

$$
\begin{aligned}
\frac{\mathrm{d} \Sigma}{\mathrm{d} \Omega}= & \frac{2 \mathrm{~N}_{\mathrm{d}} \mathrm{b}^{2} \mathrm{c}^{2} \pi^{2} \mathrm{R}^{4}}{\mathrm{Q}^{2} \mathrm{~V}_{\mathrm{M}}^{2}} . \\
& \cdot\left[(2 \mathrm{HQ} \cdot \operatorname{Si}(2 \mathrm{HQ})+\cos (2 \mathrm{HQ})-1) \cdot\left(1-\frac{\mathrm{R}^{2} \mathrm{Q}^{2}}{4}\right)+\left(1-\frac{\sin (2 \mathrm{HQ})}{2 \mathrm{HQ}}\right) \cdot \frac{\mathrm{R}^{2} \mathrm{Q}^{2}}{4}\right]
\end{aligned}
$$

Für im Vergleich zum Radius lange Zylinder $(\mathrm{H} \gg \mathrm{R})$ wird in der Guinier-Näherung $(\mathrm{QR} \ll 1)$ das Streuverhalten $(\mathrm{HQ} \gg 1)$ approximiert:

$$
\frac{2 \mathrm{HQ} \cdot \mathrm{Si}(2 \mathrm{HQ})+\cos (2 \mathrm{HQ})-1}{\mathrm{Q}^{2}} \approx \frac{\pi \mathrm{H}}{\mathrm{Q}}
$$

In dieser Näherung erhält man das Streuverhalten von Zylindern mit der Streulängendichtedifferenz $\Delta \rho^{2}=b^{2} c^{2} / V_{M}^{2} z u$ :

$$
\frac{\mathrm{d} \Sigma}{\mathrm{d} \Omega}=\frac{\pi \cdot 2 \mathrm{NH} \cdot\left(\pi \mathrm{R}^{2}\right)^{2} \cdot \Delta \rho^{2}}{\mathrm{Q}} \cdot \exp \left(-\frac{\mathrm{Q}^{2} \mathrm{R}^{2}}{4}\right)
$$

Für kleine Radien folgt der Streuquerschnitt dem $Q^{-1}-$ Verhalten linienhafter Streuobjekte:

$$
\frac{\mathrm{d} \Sigma}{\mathrm{d} \Omega}=\pi \cdot 2 \mathrm{NH} \cdot\left(\pi \mathrm{R}^{2}\right)^{2} \cdot \frac{\Delta \rho^{2}}{\mathrm{Q}}
$$




\section{Beschreibung der Experimente}

Die in den vorangegangenen Kapiteln entwickelten Modellvorstellungen zur Segregation von Wasserstoff und Deuterium an Versetzungen wurden experimentell überprüft. Im Folgenden werden die verwendeten Methoden beschrieben und erste Ergebnisse vorgestellt.

Die Präparation von Proben mit hoher Dichte an Stufenversetzungen und deren Charakterisierung wird in Kapitel 4.1 zusammen mit der Methode der elektrochemischen Beladung dargestellt. Untersuchungen der Löslichkeit von Wasserstoff und Deuterium an Versetzungen und deren Ergebnisse werden in Kapitel 4.2 beschrieben. Die Methode der Kleinwinkelneutronenstreuung ist für diese Arbeit von zentraler Bedeutung und wird in Kapitel 4.3 vorgestellt.

\subsection{Probenpräparation}

Palladium der Wielandt GmbH (Pforzheim) mit einer Reinheit von 99,95 \% wurde als Walzblech mit einer Dicke von $1 \mathrm{~mm}$ als Probenmaterial verwendet. Die weitere Behandlung der Proben wird im Folgenden dargestellt.

Die Proben wurden auf Verunreinigungen durch Sauerstoff und Kohlenstoff untersucht. Der Sauerstoffgehalt konnte mittels Trägergas-Schmelzextraktion ${ }^{3} \mathrm{zu} 16 \pm 3 \mu \mathrm{g} / \mathrm{g}$ bestimmt werden. Eine Verbrennungsanalyse ${ }^{4}$ ergab eine Kohlenstoffkonzentration von $23 \pm 8 \mu \mathrm{g} / \mathrm{g}$. Die entsprechenden Atomverhältnisse sind in Tabelle 4.1 aufgeführt.

Tabelle 4.1: $\quad$ Sauerstoff- und Kohlenstoffgehalt des verwendeten Palladiums

\begin{tabular}{|c|c|}
\hline Element & Konzentration \\
\hline Sauerstoff & $(1,1 \pm 0,2) \cdot 10^{-4} \mathrm{O} / \mathrm{Pd}$ \\
\hline Kohlenstoff & $(2,0 \pm 0,7) \cdot 10^{-4} \mathrm{C} / \mathrm{Pd}$ \\
\hline
\end{tabular}

\subsubsection{Elektrochemische Beladung}

Es gibt grundsätzlich zwei Methoden, eine Probe mit Wasserstoff zu beladen. Zum einen kann man dem Sievertsschen Gesetz (Gleichung 2.7) entsprechend durch den Gleichgewichtspartialdruck von Wasserstoff in der Gasphase eine bestimmte Konzentration im Metall-Wasserstoffsystem einstellen. Allerdings ist die Sievertskonstante meist nur ungenügend bekannt, so dass die Bestimmung der Konzentration gasvolumetrisch erfolgt, wie in Kapitel 4.2.1 beschrieben ist.

\footnotetext{
${ }^{3}$ Dr. Grallath, Max-Planck-Institut für Metallforschung, Stuttgart

${ }^{4}$ Herr Meyer, Max-Planck-Institut für Metallforschung, Stuttgart
} 
Eine definierte Beladung mit Wasserstoff ist auch elektrochemisch möglich, wenn es einen geeigneten Elektrolyten für die erforderliche Temperatur gibt. Als Elektrolyt wurde 1-normale Schwefelsäure $\mathrm{H}_{2} \mathrm{SO}_{4}$ verwendet, die auch in ihrer deuterierten Form als $\mathrm{D}_{2} \mathrm{SO}_{4}$ zur Verfügung steht. Durch die Wahl des Elektrolyten kann gezielt mit Wasserstoff oder Deuterium beladen werden. Hierbei ist allerdings zu beachten, dass sowohl schweres Wasser $\mathrm{D}_{2} \mathrm{O}$ als deuterierte Schwefelsäure $\mathrm{D}_{2} \mathrm{SO}_{4}$ hygroskopisch sind. Bei einer Verunreinigung des deuterierten Elektrolyten mit Wasserstoff löste sich dieser bevorzugt in der Probe. Eine Beladung mit Deuterium ist daher nur unter Luftabschluß möglich (Abbildung 4.1).

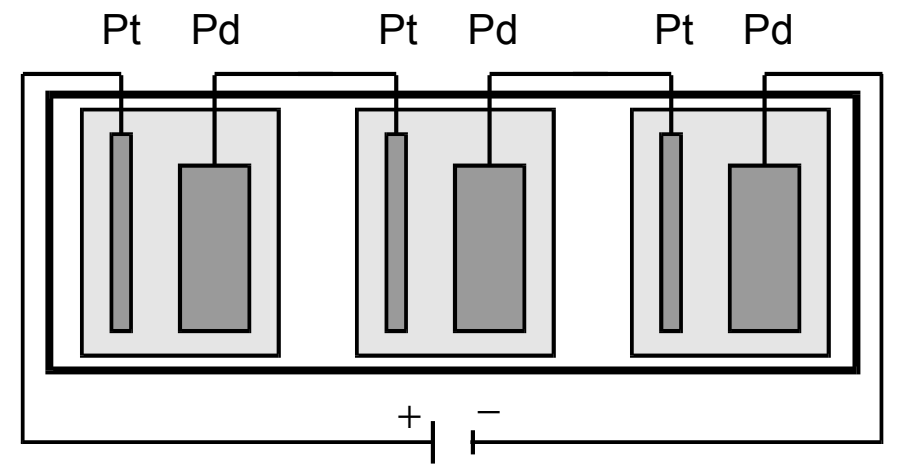

Abbildung 4.1: $\quad$ Skizze der Beladungsappartur. Die zu einem Probensatz gehörenden Proben befinden sich mit einer Gegenelektrode aus Platin in jeweils einer mit Elektrolyt gefüllten Zelle und sind in Reihe geschaltet. Der gesamte Aufbau ist luftdicht abgeschlossen.

Die Probe dient im Elektrolyten als Kathode. Bei der Beladung mit Wasserstoff läuft die folgende Reaktion an der Probenoberfläche ab:

$$
\mathrm{Pd}+\mathrm{H}_{3} \mathrm{O}^{+}+\mathrm{e}^{-} \rightarrow \mathrm{Pd} \mathrm{H}+\mathrm{H}_{2} \mathrm{O}
$$

An der Gegenelektrode aus Platin findet die Elektrolyse von Wasser statt:

$$
\mathrm{Pt}+6 \cdot \mathrm{H}_{2} \mathrm{O} \rightarrow \mathrm{Pt}+\mathrm{O}_{2}+4 \cdot \mathrm{H}_{3} \mathrm{O}^{+}+4 \mathrm{e}^{-}
$$

Die transportierte Ladung $\Delta \mathrm{Q}$ ergibt sich aus den experimentell gut zugänglichen Größen Strom I und Zeit $\Delta$ t.

$$
\Delta \mathrm{Q}=\int \mathrm{I} \mathrm{dt}
$$


Für einen konstanten Strom $\mathrm{I}_{0}$ vereinfacht sich die Gleichung $\mathrm{zu}$ dem Produkt $\mathrm{I}_{0} \cdot \Delta \mathrm{t}$. Die Menge atomaren Wasserstoffes, die an der Probenoberfläche entsteht, lässt sich nach dem Gesetz von Faraday berechnen:

$$
\Delta \mathrm{n}_{\mathrm{H}}=\frac{\Delta \mathrm{Q}}{\mathrm{q}_{\mathrm{e}} \cdot \mathrm{N}_{\mathrm{A}}}=\frac{\Delta \mathrm{Q}}{\mathrm{F}}
$$

Dabei bezeichnet $\mathrm{q}_{\mathrm{e}}$ die Elementarladung, $\mathrm{N}_{\mathrm{A}}$ die Avogadrokonstante und $\mathrm{F}$ die sich daraus ergebende Faradaykonstante. Die Konzentration in der Probe erhält man aus dem Molenbruch von Wasserstoff und Metall unter Verwendung der Probenmasse $m_{\text {Probe }}$ und der Molmasse $\mathrm{m}_{\mathrm{Pd}}$ des Palladiums:

$$
\Delta \mathrm{c}_{\mathrm{H}}=\frac{\Delta \mathrm{n}_{\mathrm{H}}}{\mathrm{n}_{\mathrm{Pd}}}=\frac{\mathrm{I}_{0} \cdot \Delta \mathrm{t}}{\mathrm{q}_{\mathrm{e}} \cdot \mathrm{N}_{\mathrm{A}}} \cdot \frac{\mathrm{m}_{\mathrm{Pd}}}{\mathrm{m}_{\text {Probe }}}
$$

Der an der Probenoberfläche entstandene Wasserstoff wird in der Probe durch Diffusion verteilt, die somit eine maximale Stromdichte bestimmt. Bei höheren Stromdichten kann der Wasserstoff nicht mehr vollständig von der Probe aufgenommen werden. Für die Beladung mit Wasserstoff oder Deuterium wurden deshalb Stromdichten unterhalb von $0,3 \mathrm{~mA} / \mathrm{cm}^{2}$ gewählt, bei denen eine vollständige Aufnahme des Wasserstoffes gewährleistet ist.

Die Entladung einer Probe erfolgt bereits passiv an Luft. Dieser insbesondere bei massiven Proben langsame Vorgang kann durch anodische Polarisation der Probe im Elektrolyten beschleunigt werden. Im Gegensatz zur Beladung, die bei konstantem Strom durchgeführt wird, erfolgt die Entladung potentiostatisch bei $800 \mathrm{mV}$. Dieser Wert liegt unterhalb des chemischen Potentials aller unerwünschten Reaktionen wie beispielsweise der Oxidation der Probe.

\subsubsection{Versetzungsbildung durch Phasenseparation}

Interstitiell gelöster Wasserstoff führt zu einer Aufweitung des Metallgitters, die auch durch das positive Vorzeichen des partiellen Atomvolumens $\overline{\mathrm{V}}$ von Wasserstoff in Metallen zum Ausdruck kommt. Wird durch Beladung einer Palladiumprobe die maximale Löslichkeit der $\alpha$-Phase überschritten, so scheidet sich die Hochkonzentrationsphase ( $\beta$ ) aus. Der Volumenmisfit der $\beta$-Phase von ca. $16 \%$ zur $\alpha$-Phase (Kapitel 2.1) führt zu großen Spannungen, die durch den Ausstoß eines prismatischen Ringes aus Zwischengitteratomen abgebaut werden können. Makenas und Birnbaum haben diese extrinsiche Versetzungsringe durch elektronenmikroskopische Untersuchungen an Niob direkt nachgewiesen [Mak80]. Ein solcher Versetzungsring, wie er in Abbildung 4.2 skizziert ist, stellt eine zusätzliche Gitterebene dar. Die Ausscheidung der $\beta$-Phase erzeugt daher einen hohen Anteil an Stufenversetzungen.

Die Beladung von Palladium mit Wasserstoff bis in das Zweiphasengebiet und anschließende Entladung soll im Folgenden als Zyklieren bezeichnet werden. 
Anderton und Mitarbeiter haben eine Abhängigkeit der mechanischen Eigenschaften von der maximalen Wasserstoffkonzentration beim Zyklieren in Palladium nachgewiesen [And96]. Der Anstieg der mechanischen Härte mit dem Grad des Zyklierens lässt auf eine entsprechende Zunahme der Versetzungsdichte schließen. Aufgrund von Untersuchungen der Löslichkeit von Wasserstoff in Palladium hat Flanagan angenommen, dass die Versetzungsdichte in zykliertem Material höher als in gewalztem ist. Die höchste Versetzungsdichte besass jedoch Material, das zunächst gewalzt und dann zykliert wurde. In zyklierten Proben konnte auch elektronenmikroskopisch eine hohe Versetzungsdichte nachgewiesen werden [Jam76]. Derartige Untersuchungen werden in Kapitel 4.1.3 vorgestellt.

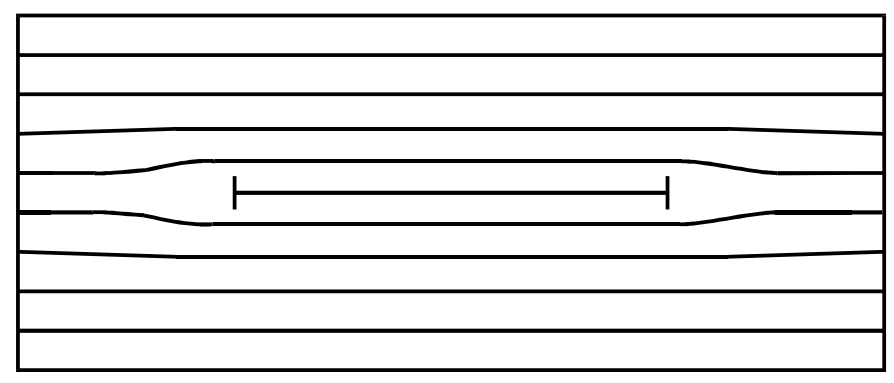

Abbildung 4.2: $\quad$ Skizze eines prismatischen Versetzungsringes und der benachbarten Gitterebenen.

Die Proben wurden, wie in Kapitel 4.1.1 beschrieben, elektrochemisch in 1-normaler Schwefelsäure etwa einen Tag bei Raumtemperatur mit einer Stromdichte von ca. $20 \mathrm{~mA} / \mathrm{cm}^{2}$ beladen, bei der die Aufnahme des Wasserstoffes durch die Proben besonders bei hohen Konzentrationen unvollständig ist. Die erreichte Konzentration wurde daher durch Wiegen bestimmt und mit $0,73 \mathrm{H} / \mathrm{Pd}$ angegeben. Folglich wurde das Zweiphasengebiet vollständig durchquert. Anschließend erfolgte eine potentiostatische Entladung bei $800 \mathrm{mV}$.

Für die Experimente der Neutronenstreuung wurden mit diesem Verfahren zwei Probensätze bestehend aus drei Einzelproben präpariert. Das Zyklieren erfolgte einmal direkt an den Walzblechen und einmal nach einer zweistündigen Glühung bei $1150^{\circ} \mathrm{C}$ im Vakuum mit anschließender Ofenabkühlung. Die Probensätze werden im Folgenden als gewalzt und zykliert und als geglüht und zykliert bezeichnet.

\subsubsection{Charakterisierung der Versetzungsdichte}

Für die Bestimmung der Versetzungsdichte $\zeta$ stand ein Elektronenmikroskop Philips EM420ST zur Verfügung, welches mit einer Beschleunigungsspannung von $120 \mathrm{kV}$ betrieben wurde. Die elektronenmikroskopischen Proben wurden aus jeweils einer geglühten und einer versetzungsreichen Probe präpariert, die zuvor für die SANS-Untersuchungen verwendet worden waren. Aus dem Material wurden zuerst mittels Funkenerosion Scheiben der Dicke $200 \mu \mathrm{m}$ geschnitten, dann daraus Plättchen mit einem Durchmesser von 2,3 mm gestanzt und 
anschließend mechanisch auf eine Dicke von ca. $100 \mu \mathrm{m}$ geschliffen. Das elektrochemische Dünnen nach dem Jet-Polishing-Verfahren erfolgte mit Hilfe eines Tenupol-3 der Fa. Struers bei einer Temperatur von $1{ }^{\circ} \mathrm{C}$, minimaler Flussrate und einer Spannung von $40 \mathrm{~V}$ unter Verwendung eines Elektrolyten aus $800 \mathrm{ml}$ Essigsäure und $200 \mathrm{ml}$ Perchlorsäure.

Abbildung 4.3 zeigt elektronenmikroskopische Aufnahmen der in Kapitel 4.1.2 als geglüht und zykliert bezeichneten Probe und einer geglühten Probe aus demselben Material. Die Abbildungsbedingungen wurden so gewählt, dass ein Versetzungskontrast erzeugt wurde. Die Versetzungsdichte $\zeta$ ist als Gesamtlänge der Versetzungslinie pro Einheitsvolumen definiert. Sie entspricht aufgrund der kleinen Probendicke im durchstrahlten Bereich dem Verhältnis der Durchstoßpunktezahl von Versetzungslinien zur jeweiligen Fläche. Da im Elektronenmikroskop nicht alle Versetzungen gleichzeitig abgebildet werden können, stellt die so ermittelte Versetzungsdichte eine untere Abschätzung dar. Die Genaugigkeit der Methode ist erfahrungsgemäß auf eine Größenordnung beschränkt.

Tabelle 4.2: $\quad$ Versetzungdichten der geglühten sowie der geglühten und dann zyklierten Probe.

\begin{tabular}{|l|c|}
\hline \multicolumn{1}{|c|}{ Probe } & Versetzungsdichte $\zeta$ \\
\hline Pd, geglüht & $\sim 10^{8} \mathrm{~cm}^{-2}$ \\
\hline Pd, geglüht und zykliert & $\sim 5 \cdot 10^{11} \mathrm{~cm}^{-2}$ \\
\hline
\end{tabular}

Die Versetzungsdichte der geglühten Probe besitzt eine für gut erholte Metalle typische Größenordnung. Der Wert für die geglühte und dann zyklierte Probe liegt in dem Bereich stark verformter Metalle und stimmt mit den von Heuser und King [Heu97] angegebenen Werten überein.

Ein Beispiel für die Versetzungsstruktur eines zyklierten Palladiumwalzbleches zeigt Abbildung 4.4. Bei dieser Probe wurde ein deutlich höherer Walzgrad als bei dem zuvor beschriebenen Material erreicht. Dies erklärt möglicherweise die hier im Vergleich zur geglühten und dann zyklierten Probe zusätzlich ausgebildeten Zellstrukturen.

Auf eine Bestimmung des Burgersvektors zur Unterscheidung zwischen Stufen- und Schraubenversetzungen wurde verzichtet, weil durch Zyklieren, wie in Kapitel 4.1.2 beschrieben, fast nur Stufenversetzungen erzeugt werden. 

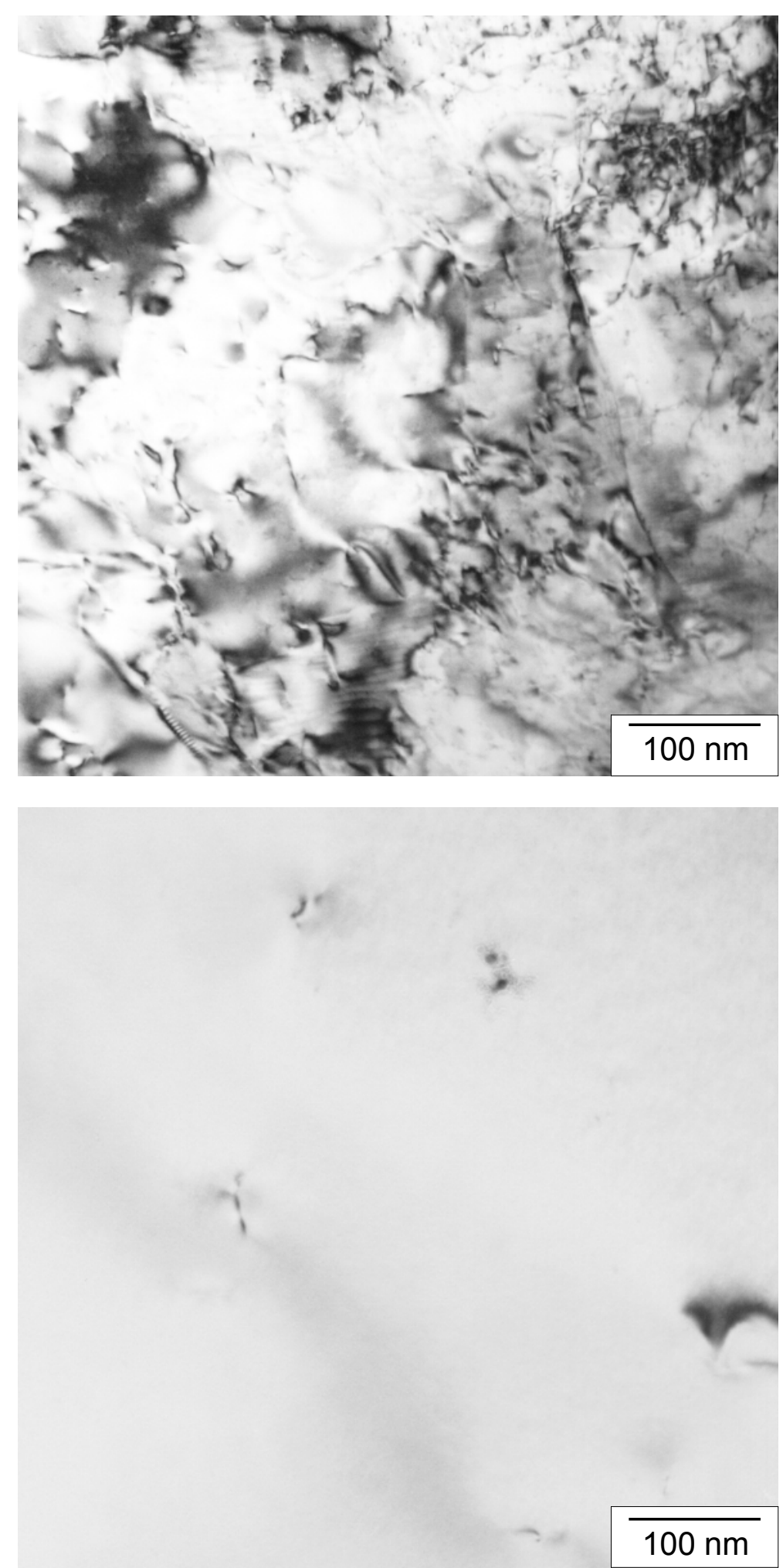

Abbildung 4.3: $\quad$ TEM Abbildungen (Hellfeld) der Versetzungsstruktur von Palladium.

Oben: geglühte und dann zyklierte Probe mit hoher Versetzungsdichte. Unten: geglühte Probe mit geringer Versetzungsdichte. 


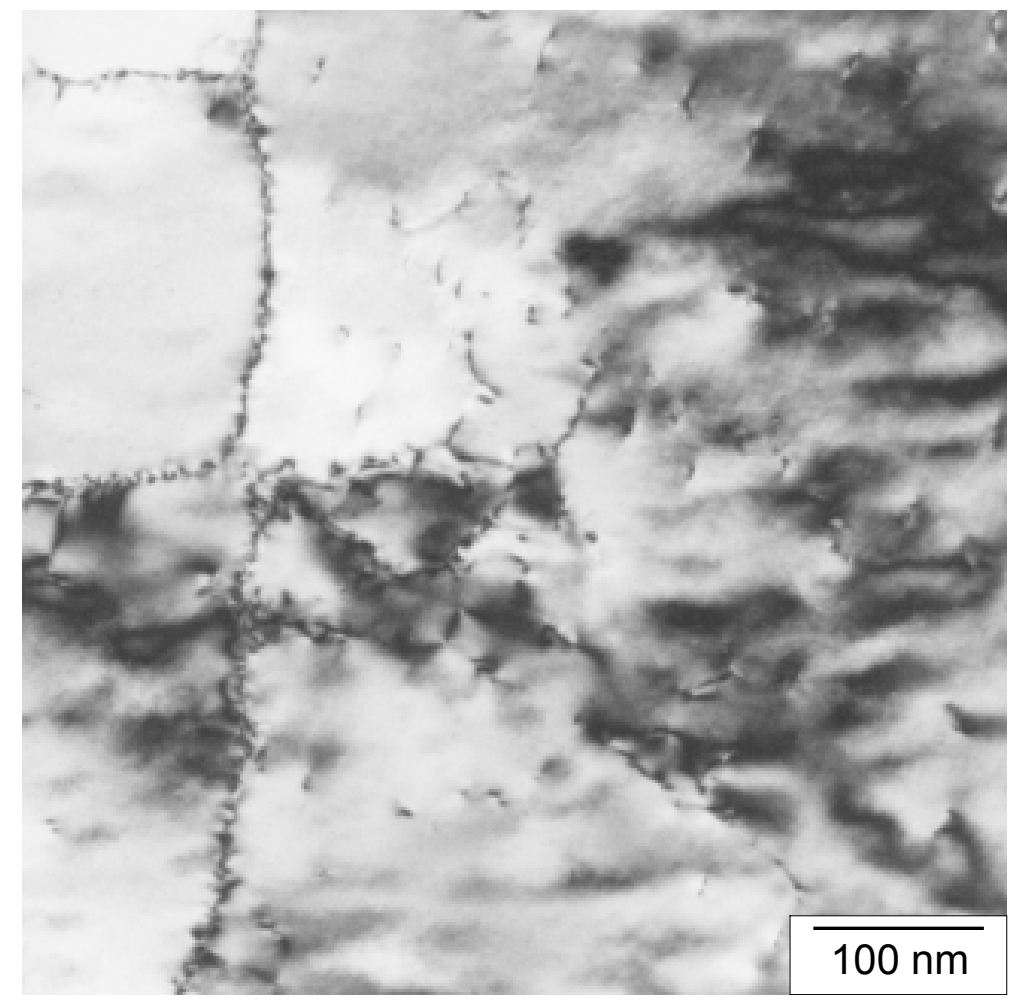

Abbildung 4.4: TEM Abbildungen (Hellfeld) der Versetzungsstruktur von zykliertem Palladiumwalzblech mit hohem Walzgrad.

\subsection{Bestimmung der segregierten Wasserstoffmenge}

Die Löslichkeit von Wasserstoff in Metallen wird durch die Segregation an Versetzungen beeinflusst. Allgemein nimmt ein Festkörper bei gegebenem Wasserstoffpartialdruck $\mathrm{p}_{\mathrm{H}_{2}}$ und gegebener Temperatur $\mathrm{T}$ eine definierte Menge $\mathrm{c}_{\mathrm{H}}$ an Wasserstoff auf, die als Löslichkeit bezeichnet wird. Sie setzt sich nach Gleichung 2.3 aus der partiellen Löslichkeit $\mathrm{c}_{\mathrm{f}}$ des „freien“ Wasserstoffs im idealen Gitter und der zusätzlichen Löslichkeit $\Delta \mathrm{c}$ im Dilatationsfeld der Versetzung zusammen.

$$
\mathrm{c}_{\mathrm{H}}=\mathrm{c}_{\mathrm{f}}+\Delta \mathrm{c}
$$

Dies entspricht einer Erhöhung der Löslichkeit durch den Einfluss von Versetzungen.

Die Löslichkeit ist eine Gleichgewichtsgröße und das System wird daher nach Gleichung 2.22 insbesondere durch das chemische Potential $\mu_{\mathrm{f}}$ des „freien“ Wasserstoffs (G1. 2.23) beschrieben. Dementsprechend ist der Wasserstoffpartialdruck $\mathrm{p}_{\mathrm{H}_{2}}$ eine eindeutige Funktion der partiellen Löslichkeit $\mathrm{c}_{\mathrm{f}}$ (Sievertssches Gesetz, Gl. 2.7) und hängt nicht von der Erhöhung $\Delta \mathrm{c}$ der Löslichkeit $\mathrm{ab}$. Folglich gehört zu einer unverformten Probe, für die $\Delta \mathrm{c}=0$ angenommen werden kann, und zu einer versetzungsreichen Probe derselbe Wasserstoffpartialdruck, wenn 
sie dieselbe Konzentration $\mathrm{c}_{\mathrm{f}}$ besitzen. Die aufgenommene Wasserstoffmenge hingegen ist im Fall der verformten Probe um $\Delta c$ erhöht.

In dieser Arbeit wird die Löslichkeitserhöhung $\Delta \mathrm{c}$ in Abhängigkeit von der Konzentration $\mathrm{c}_{\mathrm{f}}$ an „freiem“ Wasserstoff mit der Methode der Gasvolumetrie ermittelt.

\subsubsection{Die Methode der Gasvolumetrie}

Eine Probe sorbiert solange Wasserstoff, bis sie sich mit der Gasphase im Gleichgewicht befindet. Mit der Methode der Gasvolumetrie wird der Gleichgewichtswasserstoffpartialdruck direkt und die aufgenommene Wasserstoffmenge aus dem Druckabfall bestimmt.

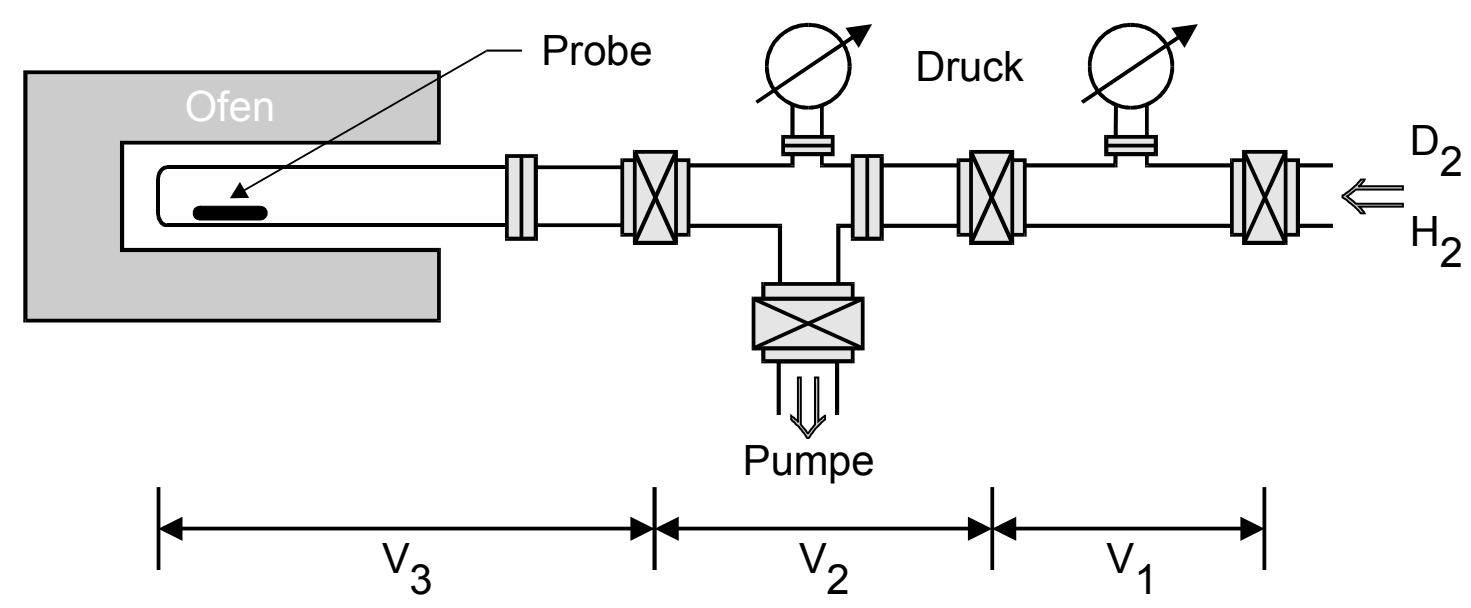

Abbildung 4.5: $\quad$ Schematische Übersichtsskizze des Apparaturaufbaus zur Gasvolumetrie

Die verwendete Apparatur ist in Abbildung 4.5 skizziert. Die Probe befindet sich in einem Quarzrohr, dessen Temperatur mit einem Ofen kontrolliert werden kann. Der gesamte Rezipient wird über eine Turbomolekularpumpe TCP 121 der Firma Balzers und eine Drehschieberpumpe DUO 016 desselben Herstellers evakuiert. Der Restgasdruck wurde mit einem Massenspektrometer zu ca. $10^{-6}$ mbar bestimmt, wobei das Restgas aus Wasserstoff, Kohlenmonoxid und geringen Mengen Sauerstoff besteht. Der Druckverlauf wurde über MKS Baraton Druckmessdosen aufgezeichnet.

Die Kinetik der Wasserstoffaufnahme wird einerseits durch die Diffusion innerhalb der Probe und andererseits durch die Dissoziation des $\mathrm{H}_{2}$-Moleküls an der Oberfläche kontrolliert. Letzterer Prozess ist bei Raumtemperatur dominierend [Fla76a] und macht eine Aktivierung der Oberfläche erforderlich. Dazu wurde die Probe mechanisch geschliffen, sorgfältig mit Isopropanol im Ultraschallbad gereinigt, getrocknet und nach dem Einbau in den Rezipienten für mindestens $12 \mathrm{~h}$ bei $80^{\circ} \mathrm{C}$ evakuiert. Anschließend wurde sie kurz mit Wasserstoff begast, für 10 min einer Temperatur von $600{ }^{\circ} \mathrm{C}$ ausgesetzt und wiederum für $12 \mathrm{~h}$ evakuiert. Dieses Vorgehen stellt außerdem sicher, dass die Probe wasserstofffrei ist [Fla76a]. 


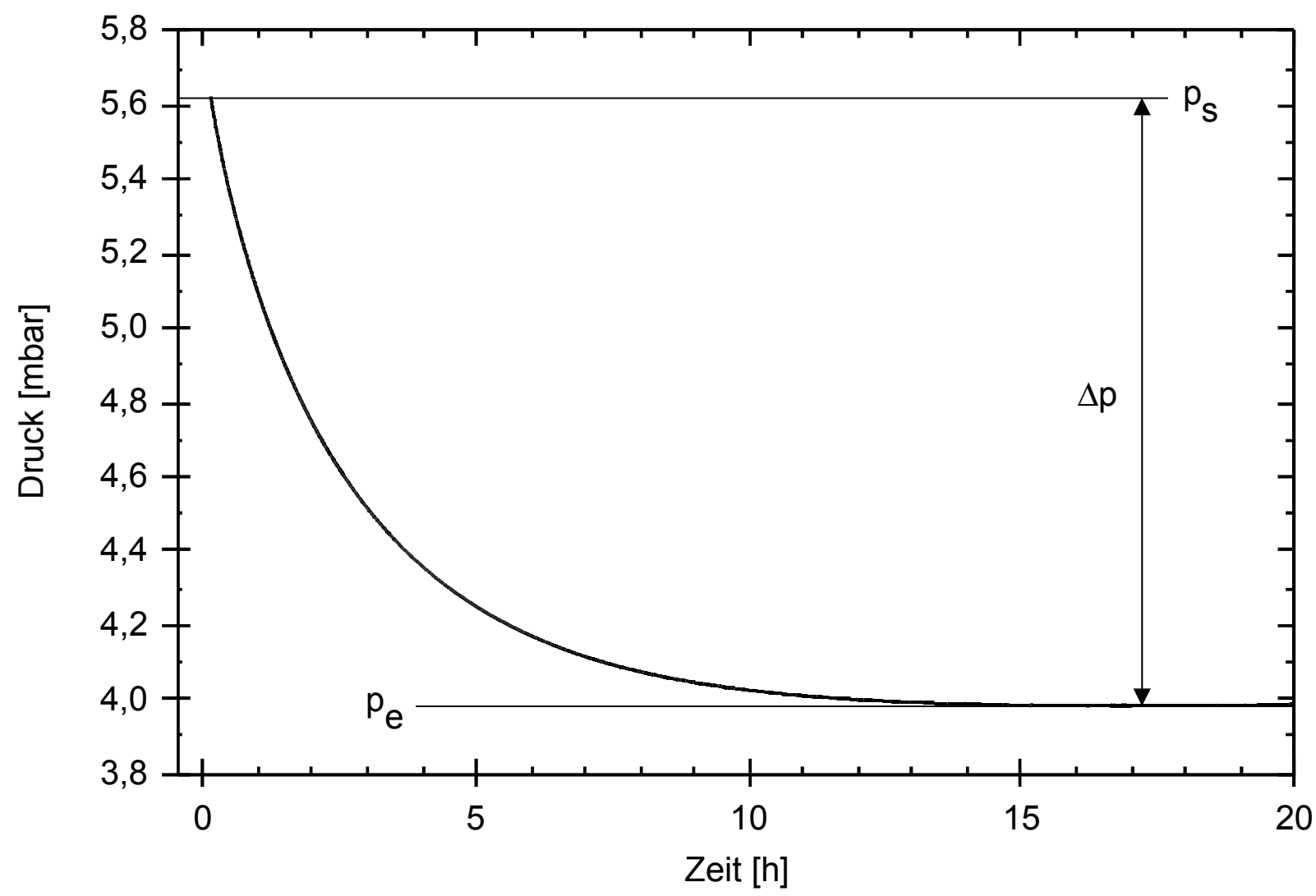

Abbildung 4.6: Beispiel einer Sorptionsmessung. Die Probe beginnt die Wasserstoffaufnahme bei einem Startdruck $\mathrm{p}_{\mathrm{s}}$ im Rezipienten und erreicht nach ca. $17 \mathrm{~h}$ den Gleichgewichtsdruck $p_{e}$, was einem Druckabfall $\Delta p$ entspricht.

Experimentell befindet sich die Probe in dem evakuierten Probenvolumen $\left(\mathrm{V}_{2}+\mathrm{V}_{3}\right.$ in Abbildung 4.5) und im Referenzvolumen $\mathrm{V}_{1}$ ist ein bestimmter Wasserstoffdruck eingestellt. Der entsprechende Startdruck $\mathrm{p}_{\mathrm{s}}$ im gesamten Rezipienten wurde direkt aus Messungen ohne Probe bestimmt. Dies berücksichtigt den Temperaturgradienten zwischen dem Probenraum im Ofen und dem Rezipienten außerhalb. Mit Öffnen des Ventils zwischen Referenz- und Probenvolumen sorbiert die Probe Wasserstoff und ein stationärer Enddruck $p_{\mathrm{e}}$ stellt sich ein. Bei $80{ }^{\circ} \mathrm{C}$ wurde das Gleichgewicht in 2 bis 20 Stunden erreicht. Abbildung 4.6 zeigt exemplarisch eine Sorptionsmessung. Als Ergebnis erhält man den Gleichgewichtswasserstoffpartialdruck $\mathrm{p}_{\mathrm{H}_{2}}$ und die Änderung $\Delta \mathrm{c}$ der Wasserstoffkonzentration für die eingestellte Temperatur T:

$$
\begin{aligned}
\mathrm{p}_{\mathrm{H}_{2}} & =\mathrm{p}_{\mathrm{e}} \\
\Delta \mathrm{c} & =\frac{\Delta \mathrm{n}_{\mathrm{H}}}{\mathrm{n}_{\mathrm{Pd}}}=\frac{2 \cdot \frac{\mathrm{V}}{\mathrm{RT}} \cdot\left(\mathrm{p}_{\mathrm{e}}-\mathrm{p}_{\mathrm{s}}\right)}{\frac{\mathrm{m}_{\text {Probe }}}{\mathrm{m}_{\text {Pd }}}}
\end{aligned}
$$


Dabei bezeichnet $\mathrm{V}$ das Volumen des Rezipienten, $\mathrm{R}$ die allgemeine Gaskonstante, $\mathrm{m}_{\text {Probe }}$ die Masse der Palladiumprobe und $\mathrm{m}_{\mathrm{Pd}}=106,42 \mathrm{~g} / \mathrm{mol}$ die Molmasse von Palladium.

Tabelle 4.3: $\quad$ Proben für die Gasvolumetrie

\begin{tabular}{|c|c|c|}
\hline Probe & Geometrie & Masse \\
\hline Pd, unverformt & $2 \times(15 \times 15 \times 0,79) \mathrm{cm}^{3}$ & $4256,7 \mathrm{mg}$ \\
\hline Pd, verformt & $2 \times(15 \times 15 \times 0,75) \mathrm{cm}^{3}$ & $4078,9 \mathrm{mg}$ \\
\hline
\end{tabular}

Beide Probensätze bestehen aus dem in Kapitel 4.1 beschriebenen Material und wurden bei $1150{ }^{\circ} \mathrm{C}$ geglüht. Die versetzungsreiche Probe wurde anschließend in der Apparatur gasvolumetrisch zykliert. So konnte die Aktivierungsglühung vermieden werden, die sonst zu Erholung geführt hätte. Die durch das Zyklieren erreichte Versetzungsdichte $\zeta$ ist abhängig von der maximalen Deuteriumkonzentration [And96], die bei der verformten Probe $\approx 0,5 \mathrm{D} / \mathrm{Pd}$ betragen hat. Die Probe wurde also fast vollständig in die $\beta$-Phase umgewandelt. Eine signifikante Reduktion der Versetzungsdichte $\zeta$ durch Erholung bei $80^{\circ} \mathrm{C}$ wurde bei temperaturabhängigen Untersuchungen [Kir81c, Fla87] nicht gefunden.

\subsubsection{Isothermen für Wasserstoff und Deuterium}

Ziel der gasvolumetrischen Messungen ist die Bestimmung der Löslichkeitserhöhung $\Delta \mathrm{c}$ zwischen einer verformten und einer unverformten Probe in Abhängigkeit von der Konzentration $\mathrm{c}_{\mathrm{f}}$ für beide Isotope Wasserstoff und Deuterium. Einerseits wird so der an den Versetzungen segregierte Wasserstoff quantitativ ermittelt. Einflüsse anderer Elemente der Realstruktur wie Korngrenzen oder Leerstellen sind nicht zu beachten [Fla76a]. Außerdem ermöglichen die Messungen einen direkten Vergleich zwischen den Isotopen.

Sukzessive Wiederholung der in Kapitel 4.2.1 beschriebenen Messungen lieferten DruckKonzentrationsisothermen bei $80^{\circ} \mathrm{C}$. Insgesamt wurden vier Isothermen bestimmt: Mit Wasserstoff und Deuterium jeweils für den verformten und den unverformten Probensatz. Die Messwerte der einzelnen Sorptionsexperimente und die daran angepassten Isothermen sind in Abbildung 4.7 dargestellt. Die Auftragung des Gleichgewichtsgasdruckes erfolgt dabei gegen die Löslichkeit $\mathrm{c}=\mathrm{c}_{\mathrm{f}}+\Delta \mathrm{c}$ der Probe. 

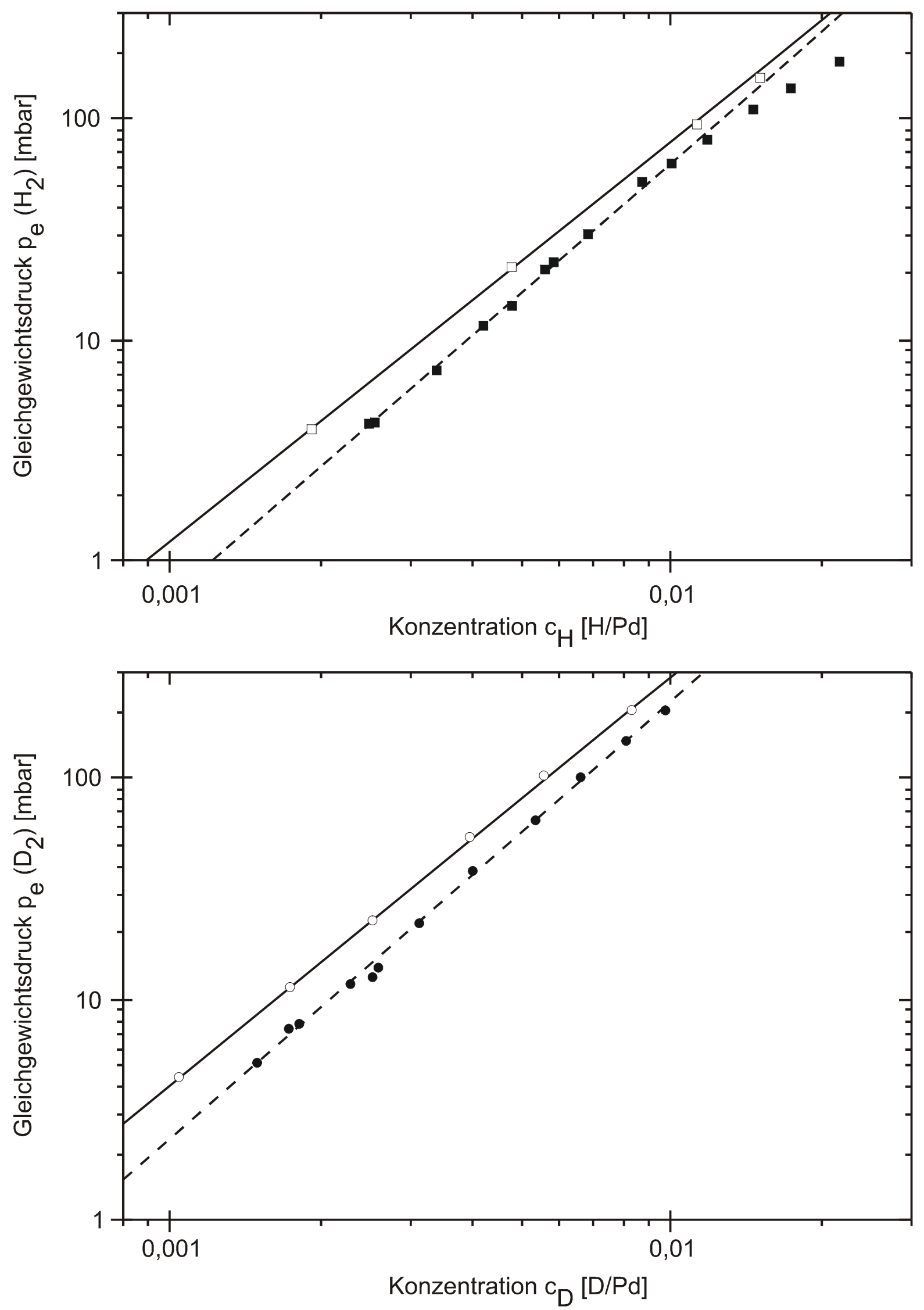

Abbildung 4.7: Druck-Konzentrationsisothermen bei $80^{\circ} \mathrm{C}$ für Wasserstoff (oben) und Deuterium (unten). Die offenen Symbole $\square \bigcirc$ beziehen sich auf die unverfomte und die gefüllten $1 \bullet$ auf die verformte Probe. Die Kurven sind eine Anpassung von Gleichung 4.9 an die Messwerte. 
Zunächst fällt auf, dass der zu einer Konzentration c gehörige Deuteriumpartialdruck höher als der entsprechende Wasserstoffpartialdruck ist. Man würde erwarten, dass das schwerere Isotop Deuterium aufgrund seiner geringeren Nullpunktsenergie [Dre76] bevorzugt in Lösung geht. Tatsächlich jedoch ist die Differenz zwischen den Nullpunktsenergien von Wasserstoff und Deuterium in der Gasphase größer als in den Oktaederplätzen des Palladiums, wie in Abbildung 4.8 skizziert ist. Dieser Zusammenhang wird auch durch die Sievertskonstante (Gl. 2.7) berücksichtigt:

$$
\mathrm{c}_{\mathrm{f}}=\exp \left(\frac{\mu_{\mathrm{H}}^{0}-\frac{1}{2} \mu_{\mathrm{H}_{2}}^{0}}{\mathrm{RT}}\right) \cdot \sqrt{\mathrm{p}_{\mathrm{H}_{2}}}
$$

Für Wasserstoff und Deuterium sind die Standardpotential $\mu^{0}$ sowohl in der Gasphase als auch in der Lösung unterschiedlich. Dies erklärt den höheren Deuteriumpartialdruck. Damit ist insbesondere noch keine Aussage über die Löslichkeitserhöhung $\Delta \mathrm{c}$ bei einer gegebenen Konzentration $\mathrm{c}_{\mathrm{f}}$ gemacht. Zusätzlich ist anzumerken, dass dem einfachen Bild von Abbildung 4.8 ein starres parabelförmiges Potential zugrunde liegt, welches die Gitterverzerrungen durch ein Zwischengitteratom nicht berücksichtigt. Eine Bestimmung des partiellen Atomvolumens $\overline{\mathrm{V}}$ ist daher nicht möglich.

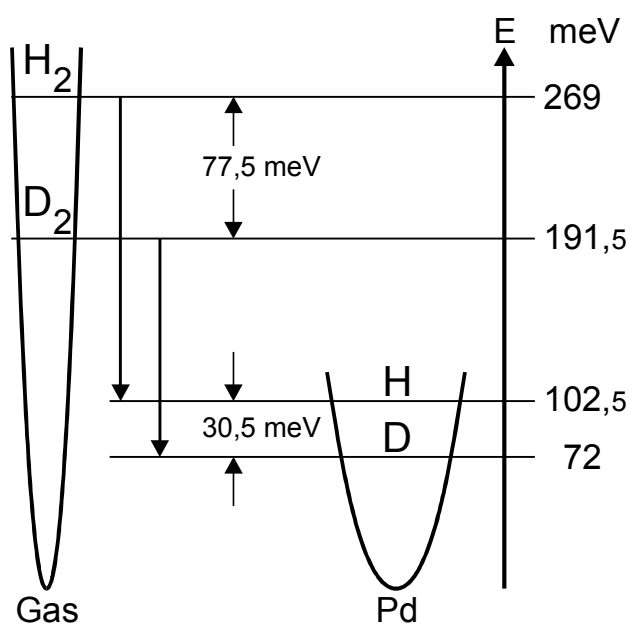

Abbildung 4.8: Nullpunktsenergien für Wasserstoff und Deuterium in der Gasphase und in den Oktaederplätzen des Palladiums [Wic78].

An die einzelnen Werte der Sorptionsmessungen in Abbildung 4.7 wurden Isothermen der Form:

$$
\log \mathrm{p}=\mathrm{A}+\mathrm{B} \cdot \log \mathrm{c}
$$


angepasst. Die Fitparameter sind in Tabelle 4.4 aufgeführt. Dabei wurden die beiden Messwerte der verformten Probe mit den höchsten Wasserstoffkonzentrationen nicht berücksichtigt, weil sie bereits zum 2-Phasengebiet abknicken.

Tabelle 4.4: $\quad$ An die Messwerte angepasste Isothermen für $80^{\circ} \mathrm{C}$ mit statistischem Fehler

\begin{tabular}{|c|c|c|}
\hline Wasserstoff & unverformt & $\log p=(5,509 \pm 0,062)+(1,804 \pm 0,026) \cdot \log \mathrm{c}$ \\
\hline Wasserstoff & verformt & $\log \mathrm{p}=(5,647 \pm 0,077)+(1,926 \pm 0,035) \cdot \log \mathrm{c}$ \\
\hline Deuterium & unverformt & $\log \mathrm{p}=(6,185 \pm 0,049)+(1,856 \pm 0,019) \cdot \log \mathrm{c}$ \\
\hline Deuterium & verformt & $\log \mathrm{p}=(6,313 \pm 0,120)+(1,978 \pm 0,050) \cdot \log \mathrm{c}$ \\
\hline
\end{tabular}

Alle Isothermen weichen systematisch vom Sievertsschen Verhalten (Gl. 4.8) ab und besitzen eine Steigung $B<2$, wie sie für beide Isothermen an der unverformten Probe zu erwarten wäre. Dieser systematische Fehler tritt sowohl für die verformte als auch für die unverformte Probe auf und muss daher bei der hier untersuchten Differenz $\Delta \mathrm{c}$ nicht berücksichtigt werden. Die Isothermen der verformten Proben verlaufen wie erwartet aufgrund der Löslichkeitserhöhung $\Delta \mathrm{c}$ jeweils steiler als die der unverformten.

Die in Tabelle 4.4 angegebenen Isothermen stimmen gut mit den bekannten Werten aus der Literatur überein, die in [Rau76] aufgeführt sind. Für das System Palladium-Deuterium wird beispielsweise bei $80^{\circ} \mathrm{C}$ eine Isotherme $\log \mathrm{p}=6,216+2 \cdot \log \mathrm{c}[\mathrm{Cle} 73]$ angegeben.

Die Erhöhung $\Delta \mathrm{c}$ der Löslichkeit wird in Kapitel 5.1 anhand der gasvolumetrisch bestimmten Isothermen für Wasserstoff und Deuterium diskutiert.

\subsection{Kleinwinkelneutronenstreuung}

Die Untersuchungen mit der Methode der Kleinwinkelneutronenstreuung (SANS) wurden am Forschungszentrum Jülich hauptsächlich am Instrument KWS1 und ergänzend an KWS2 durchgeführt. Das experimentelle Vorgehen sowie die Berechnung des makroskopischen Streuquerschnittes aus den gemessenen Intensitäten nach der Sekundärstandardmethode werden in diesem Kapitel beschrieben. Außerdem wird der Nettostreuquerschnitt eingeführt. Die weitere Auswertung und Diskussion der Ergebnisse erfolgt in Kapitel 5.2.

\subsubsection{Experimentelles Vorgehen}

Für die Neutronenstreuung wurde jeweils ein Probensatz aus drei identisch präparierten Palladiumblechen der Dimension $15 \times 15 \times 1 \mathrm{~mm}^{3}$ verwendet. Die Beladung mit Wasserstoff und Deuterium erfolgte, wie in Kapitel 4.1.1 beschrieben, elektrochemisch direkt vor den SANSMessungen bei Raumtemperatur. Die Beladungsströme lagen im Bereich von Milliampère und damit deutlich unter der kritischen Stromdichte von $0,3 \mathrm{~mA} / \mathrm{cm}^{2}$. Die entsprechenden 
Beladungszeiten waren grundsätzlich länger als die zu einer Stunde abgeschätzte Diffusionszeit von Wasserstoff für die halbe Probendicke. Nach der Beladung wurden die Proben unverzüglich in einen wasserstoffdichten Probenhalter aus Quarzglas eingebracht, der dann evakuiert wurde. Die Desorption der Proben bis zur Einstellung des Gleichgewichtpartialdrucks kann aufgrund des kleinen Volumens vernachlässigt werden. Durch Verwendung von Quarzglas mit einer Wandstärke von ca. $1 \mathrm{~mm}$ konnte eine Transmission für Neutronen besser als 0,9 erreicht werden.

Der Strahlengang der Kleinwinkelstreuapparaturen KWS1 und KWS2 des Forschungszentrums Jülich ist in Abbildung 4.9 skizziert. Beide Instrumente unterscheiden sich nur durch die Selektoren und Detektoren. Außerdem ist der Neutronenleiter zur KWS1 gerade und der zur KWS2 gekrümmt. Da im Rahmen dieser Arbeit fast alle Messungen an der KWS1 durchgeführt wurden, wird auf eine gesonderte Beschreibung dieser geringen Unterschiede verzichtet. Sie wurden bei der Eichung der gemessenen Intensitäten berücksichtigt.

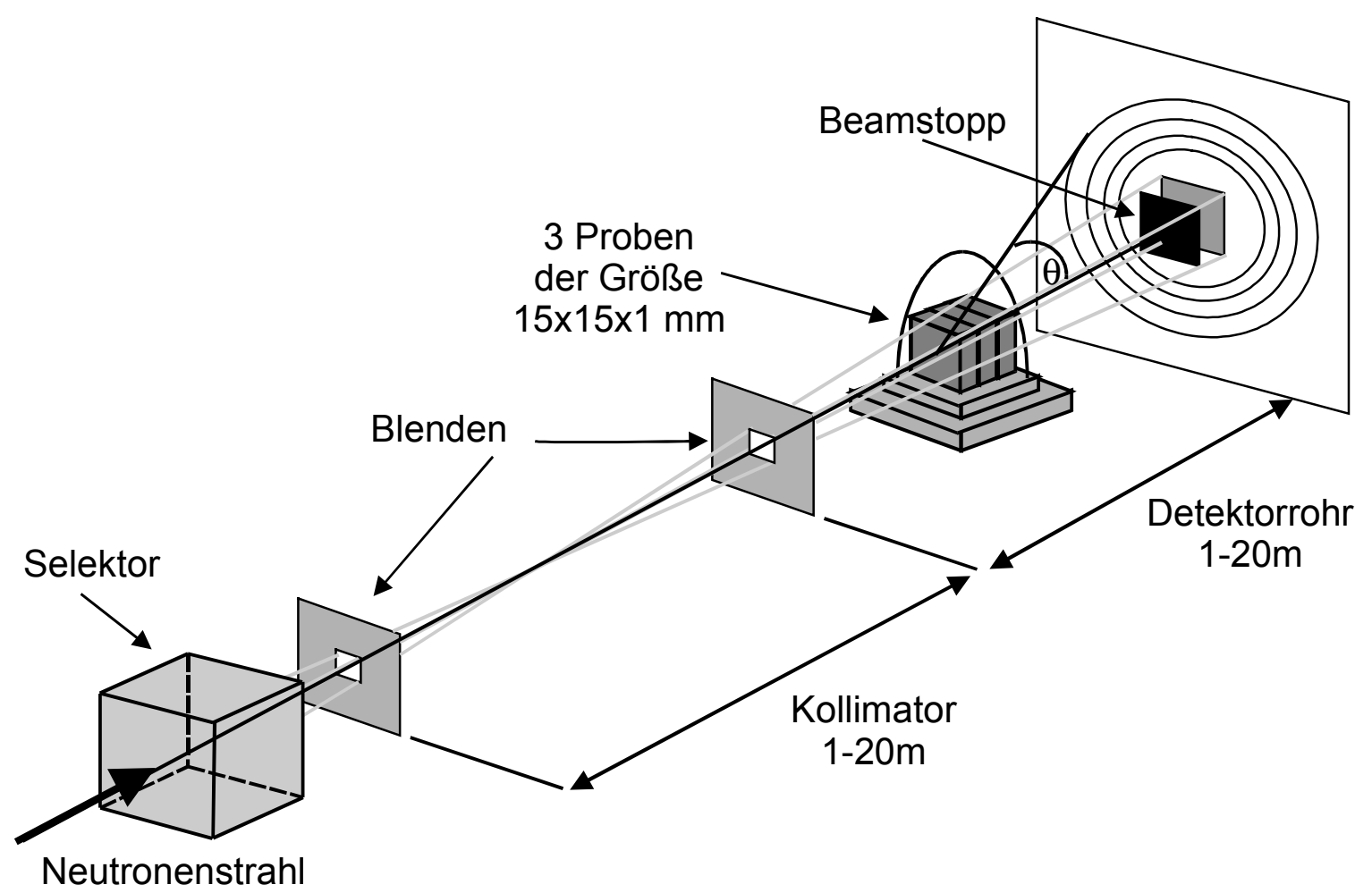

Abbildung 4.9: $\quad$ Schematische Übersichtsskizze der Neutronenkleinwinkelstreuapparatur

Die Kleinwinkelstreuappartur (Abbildung 4.9) ist evakuiert und besteht im Wesentlichen aus einer Kollimatorstrecke, der Probenkammer und dem Detektor im Detektorrohr. Der Abstand von Probe zu Detektor kann durch Verfahren des Detektors zwischen 1,25 m und $20 \mathrm{~m}$ eingestellt werden. Die Kollimatorstrecke ist ein System aus Blenden und Neutronenleitersegmenten. Das Optimum an Intensität und Auflösung wird erreicht, wenn der Abstand der beiden 
die Divergenz des Neutronenstrahls bestimmenden Kollimatorblenden dem Probe-Detektorabstand entspricht. Eine Blende ist direkt vor der Probe angeordnet und bestimmt den Strahlquerschnitt. Die zweite Kollimatorblende ist diejenige unmittelbar hinter dem letzten im Neutronenstrahl befindlichen Neutronenleitersegment. Der Primärstrahl ungestreuter Neutronen fällt so vollständig auf den in der Mitte des Detektors angebrachten Strahlfänger (Beamstopp). Ein darin eingebauter ${ }^{3} \mathrm{He}-Z$ ähler ermöglicht eine direkte Bestimmung der Transmission. Es treffen nur gestreute Neutronen auf die aktive Fläche des Detektors aus ${ }^{6}$ Li-Szintillationsglas. Eine $8 \times 8$ Photomultiplieranordnung lokalisiert das Ereignis in $64 \times 64$ Kanälen. Mit dem Geschwindigkeitsselektor wird die Wellenlänge $\lambda_{n}$ der Neutronen eingestellt. Die in dieser Arbeit gewählten Einstellungen sind in Tabelle 4.5 zusammengefasst.

Tabelle 4.5: $\quad$ Experimentelle Parameter der Kleinwinkelneutronenstreuung

\begin{tabular}{|c|c|c|c|}
\hline Neutronenwellenlänge $\lambda_{\mathrm{n}}$ & \multicolumn{3}{|c|}{$7,0 \AA \mathrm{mit} \Delta \lambda_{\mathrm{n}} / \lambda_{\mathrm{n}}=0,2$} \\
\hline Abstand Probe-Detektor & $1,25 \mathrm{~m}$ & $2 \mathrm{~m}$ & $8 \mathrm{~m}$ \\
\hline Kollimation & $2 \mathrm{~m}$ & $2 \mathrm{~m}$ & $8 \mathrm{~m}$ \\
\hline aufgenommener Q-Bereich & \multicolumn{3}{|c|}{$5 \cdot 10^{-3} \ldots 2,3 \cdot 10^{-1} \AA^{-1}$} \\
\hline Strahlquerschnitt & \multicolumn{3}{|c|}{$6 \times 6 \vee 8 \times 8 \mathrm{~mm}^{2}$} \\
\hline
\end{tabular}

Die Integrationszeit (Messzeit) wurde für jede Detektorposition so gewählt, dass der statistische Fehler unter $1 \%$ zu erwarten war. Der relative Fehler einer Stichprobe mit dem Umfang $\mathrm{N}$ verhält sich wie $1 / \sqrt{\mathrm{N}}$. Aus der integralen Zählrate $\dot{\mathrm{N}}$ des gesamten Detektors kann man die durchschnittlichen Counts $\mathrm{N}_{i}$ pro Kanal in der Zeit $\mathrm{zu} \dot{\mathrm{N}} \cdot \mathrm{t} \cdot 15 / 4096$ abschätzen. Dabei ist 4096 die Anzahl der Kanäle und 15 ergibt sich aus der radialen Mittelung, die bei einen isotropen Streuverhalten zulässig ist. Bei den Detektorabständen 1,25 m und $2 \mathrm{~m}$ waren durchschnittlich 15 .. 30 min Messzeit für die gewünschte Statistik nötig. Entsprechend hätte eine Messung mit einer Detektorposition bei $8 \mathrm{~m}$ die 16-fache Messzeit erfordert, die grundsätzlich auf 2 .. 3 Stunden gekürzt wurde. Das starke Porodverhalten der Proben bei den zu diesem Detektorabstand gehörenden kleinen Q-Werten bewirkte eine erhöhte Zählrate, so dass eine vergleichbare Statistik erreicht wurde.

\subsubsection{Berechnung des makroskopischen Streuquerschnittes}

Die Umrechnung der gemessenen Intensitäten in makroskopische Streuquerschnitte erfolgte für jede Detektorposition kanalweise nach der Sekundärstandardmethode [Pyc91, Wig87]. Dabei wurde die inkohärente Streuung von Lupolen als Sekundärstandard benutzt, um die Absolutwerte des Streuquerschnittes je Einheitsvolumen aus den Zählraten I abzuleiten. Die gemessene Intensität $\mathrm{I}_{\text {Probe+Halter }}$ der Palladiumprobe in dem oben beschriebenen Probenhalter 
wurde um ihre Transmission $\mathrm{T}_{\text {Probe }}$, die Intensität $\mathrm{I}_{\text {Halter }}$ des leeren Probenhalters und seine Transmission $\mathrm{T}_{\text {Halter }}$ sowie um den Untergrund $\mathrm{I}_{0}$ korrigiert.

$$
\frac{\mathrm{d} \Sigma}{\mathrm{d} \Omega}=\left(\mathrm{T} \cdot \mathrm{D} \cdot \frac{\mathrm{d} \Sigma}{\mathrm{d} \Omega}\right)_{\text {Lupolen }} \cdot \frac{\mathrm{A}_{\text {Lupolen }} \mathrm{L}_{\text {Probe }}^{2}}{\mathrm{~L}_{\text {Lupolen }}^{2} \mathrm{~A}_{\text {Probe }}} \cdot \frac{\mathrm{I}_{\text {Probe }+ \text { Halter }}-\mathrm{I}_{0}-\mathrm{T}_{\text {Probe }}\left(\mathrm{I}_{\text {Halter }}+\mathrm{I}_{0}\right)}{\mathrm{T}_{\text {Probe }} \mathrm{T}_{\text {Halter }} \mathrm{D}_{\text {Probe }}\langle\mathrm{I}\rangle_{\text {Lupolen }}}
$$

Dabei bezeichnet D die Dicke der jeweiligen Probe, A deren Fläche im Neutronenstrahl (Strahlquerschnitt) und L ihren Abstand zum Detektor. Die inkohärente Streuung $\langle\mathrm{I}\rangle_{\text {Lupolen }}$ wurde aus einer Messung der Lupolen Eichprobe zu jeder Kollimation bestimmt. Aus der Messung mit der kleinsten Kollimation von $2 \mathrm{~m}$ wurde außerdem die Detektorempfindlichkeit ermittelt. Eine Messung des leerem Probenhalters bei jedem Detektorabstand lieferte den Streubeitrag $\mathrm{I}_{\text {Halter }}$ des Probenhalters. Ein Vergleich mit einer Leerstrahlmessung ergab die Transmission $\mathrm{T}_{\text {Halter }}$ des Halters. Mit derselben Messung erhält man $\mathrm{T}_{\text {Probe }}$ aus dem Verhältnis zur Intensität $\mathrm{I}_{\text {Probe+Halter }}$ Die Transmission wurde immer bei dem größten Detektorabstand von $8 \mathrm{~m}$ ermittelt, bei dem das kleinste Q-Intervall auf das Zählrohr im Beamstopp trifft. Borcarbid diente als Absorber für die Bestimmung des Untergrundes $\mathrm{I}_{0}$. Der Sekundärstandard Lupolen wurde mit Vanadium geeicht [Pyc91]:

$$
\left(\mathrm{T} \cdot \mathrm{D} \cdot \frac{\mathrm{d} \Sigma}{\mathrm{d} \Omega}\right)_{\text {Lupolen }}=(7,34 \pm 0,03) \cdot 10^{-2}
$$

Diese Eichung erfolgte für jeden Kanal des Detektors. Anschließend wurde radial gemittelt, weil Palladium erwartungsgemäß ein isotropes Streubild zeigte. Ein Überstrahlen des Primärstrahles konnte durch Maskieren der Kanäle nahe dem Beamstopp ausgeschlossen werden. Am äußeren Rand des Detektors erfolgt die radiale Mittelung über eine geringere Anzahl an Kanälen, so dass der relative Fehler für die größten Q-Werte einer Detektorposition zunimmt.

Die Ergebnisse aller drei Detektorpositionen wurden zu einer Streukurve zusammengefasst (Abbildung 4.10). Aus dem Überlapp der Einzelkurven konnte ein Faktor bestimmt werden, der die Totzeit des Detektors aufgrund der hohen Zählrate der Lupolen-Eichmessung bei den kleinen Detektorabständen berücksichtigt. Ein derartiger Einfluss kann bei der 8 m-Messung ausgeschlossen werden. 


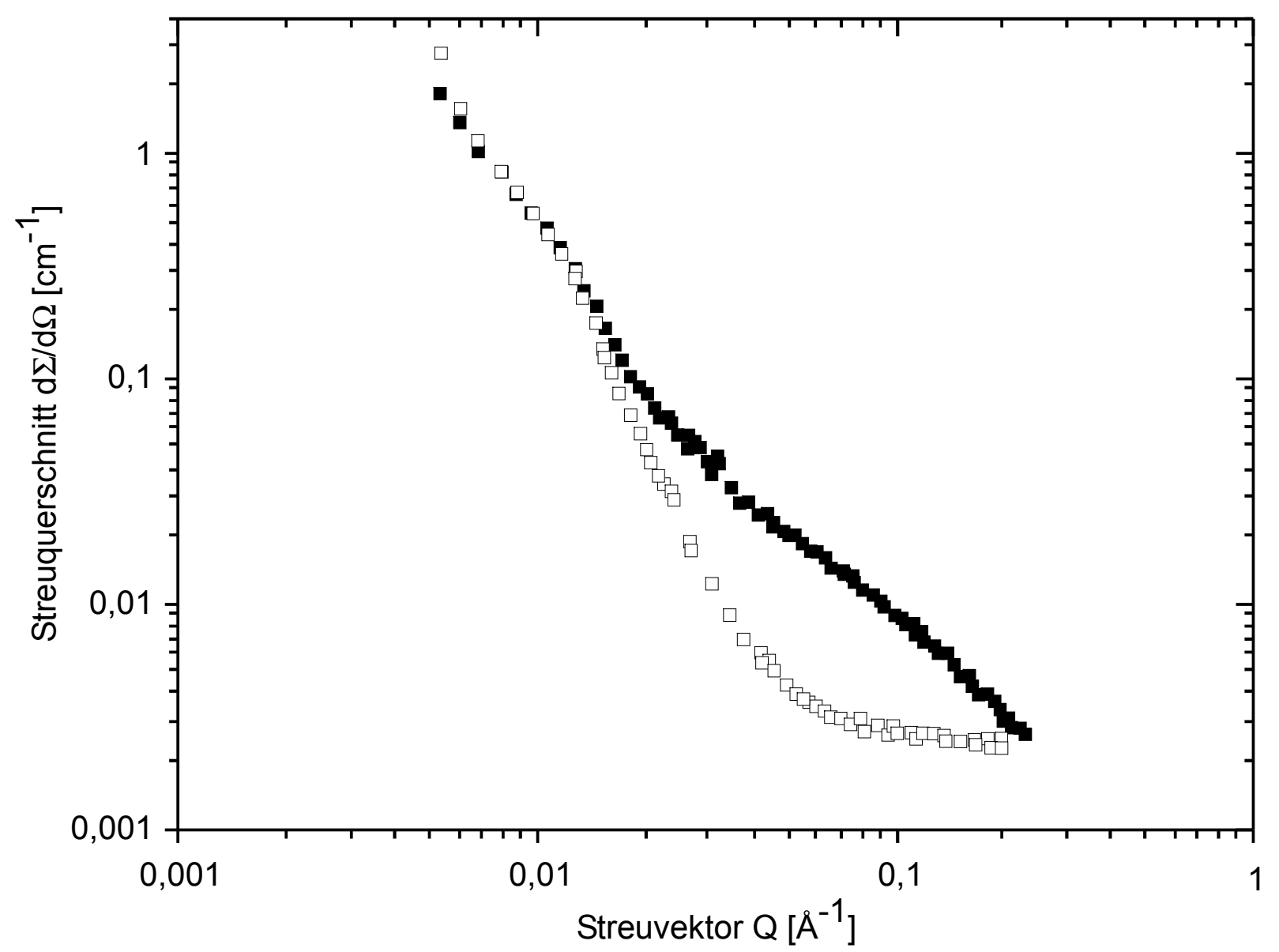

Abbildung 4.10: Streukurven einer versetzungsreichen Palladiumprobe. Der kohärente Streuquerschnitt ist doppeltlogarithmisch für den unbeladenen Zustand $(\square)$ und beladen mit $0,0105 \mathrm{H} / \mathrm{Pd}(\square)$ gegen den Streuvektor Q aufgetragen.

Abbildung 4.10 zeigt Streukurven einer versetzungsreichen Palladiumprobe. Beide Kurven besitzen für kleine Streuvektoren einen starken Anstieg, der in Kapitel 5.2.3 als Porodverhalten diskutiert wird. Für größere Streuvektoren verläuft der kohärente Streuquerschnitt der Probe im beladenen Zustand oberhalb der Kurve des unbeladenen Zustandes. Der Effekt durch die Beladung der Proben mit Wasserstoff bzw. Deuterium wird durch den Netto-Streuquerschnitt erfasst. Unter dem Netto-Streuquerschnitt soll die Differenz der Streuquerschnitte der mit einer Konzentration c beladenen und der unbeladenen $(\mathrm{c}=0)$ Probe verstanden werden:

$$
\left(\frac{\mathrm{d} \Sigma}{\mathrm{d} \Omega}\right)_{\text {netto }}=\left(\frac{\mathrm{d} \Sigma}{\mathrm{d} \Omega}\right)_{\mathrm{c}}-\left(\frac{\mathrm{d} \Sigma}{\mathrm{d} \Omega}\right)_{0}
$$

Den sich aus Abbildung 4.10 ergebende Netto-Streuquerschnitt zeigt Abbildung 5.3. Er wird in Kapitel 5.2.1 diskutiert. 
Für die Auswertung sind nur die kohärenten Streubeiträge interessant. Der inkohärenten Streubeitrag des Palladiums ist nicht im Netto-Streuquerschnitt enthalten. Dieser ist jedoch noch um die inkohärente Streuung von Wasserstoff bzw. Deuterium der Konzentration entsprechend zu vermindern:

$$
\left.\frac{\mathrm{d} \Sigma}{\mathrm{d} \Omega}\right|_{\text {inc }}=\frac{\sigma_{\text {inc }}^{(\mathrm{i})} \cdot \mathrm{c}_{\mathrm{i}} \cdot \mathrm{N}_{\mathrm{A}}}{4 \pi \cdot \Omega_{\mathrm{Pd}}}
$$

Dabei bezeichnen $\Omega_{\mathrm{Pd}}=8,852 \mathrm{~cm}^{3} / \mathrm{mol}$ das Molvolumen von Palladium und $\sigma_{\text {inc }}^{(\mathrm{i})}$ den inkohärenten Streuquerschnitt der Atomsorte i, die in Tabelle 4.6 angegeben sind.

Tabelle 4.6: $\quad$ Inkohärenter Streuquerschnitt [Sea92]

\begin{tabular}{|c|c|c|}
\hline Wasserstoff & $80,27 \cdot 10^{-24} \mathrm{~cm}^{2}$ & $\mathrm{c}_{\mathrm{i}}=\mathrm{c}_{\mathrm{H}}$ \\
\hline Deuterium & $2,05 \cdot 10^{-24} \mathrm{~cm}^{2}$ & $\mathrm{c}_{\mathrm{i}}=\mathrm{c}_{\mathrm{D}}$ \\
\hline Palladium & $0,093 \cdot 10^{-24} \mathrm{~cm}^{2}$ & $\mathrm{c}_{\mathrm{i}}=1$ \\
\hline
\end{tabular}




\section{Ergebnisse und Diskussion}

Im Folgenden werden die Ergebnisse der Gasvolumetrie und der Neutronenstreuung dargestellt und diskutiert, wobei die in Kapitel 2 entwickelte Vorstellung eines zylinderförmigen Segregationsgebietes an den Stufenversetzungen zugrunde gelegt wird. Die radiale Ausdehung des Zylinders wird gemäß Kapitel 3.3 aus den Streukurven in Abhängigkeit der Wasserstoffkonzentration quantitativ ausgewertet. Mit dem in Kapitel 2.4 vorgestellten Modell und der in Kapitel 4.1.3 bestimmten Versetzungsdichte erfolgt die Verknüpfung zwischen der Zylinderausdehnung und der an den Versetzungen segregierten Wasserstoffmenge. Letztere wird aus den Ergebnissen der Gasvolumetrie ermittelt.

Weiterhin wird gezeigt, dass Wasserstoff und Deuterium dasselbe Segregationsverhalten besitzen. Der beobachtete unterschiedliche Kontrast ihrer Streukurven wird qualitativ diskutiert. Dabei sind neben den Segregationszylindern weitere entsprechend mit Wasserstoff und Deuterium dekorierte Objekte $\mathrm{zu}$ berücksichtigen, die zu einem ausgeprägten Porodverhalten im Streubild führen.

\subsection{Gasvolumetrische Messungen}

Durch gasvolumetrische Untersuchungen der Sorption sowohl von Wasserstoff als auch von Deuterium wird die an den Versetzungen segregierte Menge $\Delta \mathrm{c}$ an erholtem und versetzungsreichem Palladium bestimmt. Dies ermöglicht, das Verhalten beider Isotope zu vergleichen. Die entsprechenden Messungen sind in Kapitel 4.2 beschrieben und Abbildung 4.7 zeigt die Ergebnisse. In Abbildung 5.1 sind die Messungen für $80^{\circ} \mathrm{C}$ als Konzentrations-Druckisothermen zusammengefasst und dargestellt. Da die zyklierten Proben eine erhöhte Löslichkeit besitzen, verlaufen ihre Isothermen oberhalb der zu den geglühten Proben gehörigen Isothermen. Außerdem sind die Deuteriumkurven entsprechend Kapitel 4.2.2 zu höheren Partialdrücken verschoben.

Für die geglühte Probe läßt sich von einem ungestörten Gitter ausgehen, so dass die gesamte von der Probe aufgenommene Menge c an Wasserstoff- bzw. Deuteriumatomen als „frei““ angenommen wird:

$$
\mathrm{c}=\mathrm{c}_{\mathrm{f}}
$$

Die zyklierte und damit versetzungsreiche Probe besitzt gemäß der Gleichungen 4.6 und 2.3 eine um $\Delta \mathrm{c}$ erhöhte Löslichkeit:

$$
\mathrm{c}=\mathrm{c}_{\mathrm{f}}+\Delta \mathrm{c}
$$


Der gasvolumetrisch bestimmte Gleichgewichtsgasdruck $\mathrm{p}$ ist durch das chemische Potential $\mu$ gegeben und damit nach dem Sievertsschen Gesetz (G1. 2.7 und 4.8) nur von der Konzentration $\mathrm{c}_{\mathrm{f}}$ der gelösten Wasserstoff- bzw. Deuteriumatome im ungestörten Gitter abhängig. Die Erhöhung $\Delta \mathrm{c}$ der Löslichkeit hat insbesondere keinen Einfluss auf den Partialdruck $\mathrm{p}$. Dementsprechend kann für jedes Isotop gemäß der Gleichungen 5.1 und 5.2 die Löslichkeitserhöhung $\Delta \mathrm{c}$ als Differenz der Konzentrations-Druckisothermen der zyklierten und der geglühten Probe bestimmt werden. Abbildung 5.1 zeigt die sich ergebenden Kurvenverläufe als Funktion des Gleichgewichtsgasdruckes. Die erwartungsgemäß (Kapitel 4.2.2) zu höheren Partialdrücken verschobene Deuteriumkurve $\Delta \mathrm{c}_{\mathrm{D}}$ läßt noch nicht auf einen Isotopeneffekt bei der Segregation an den Versetzungen schließen.

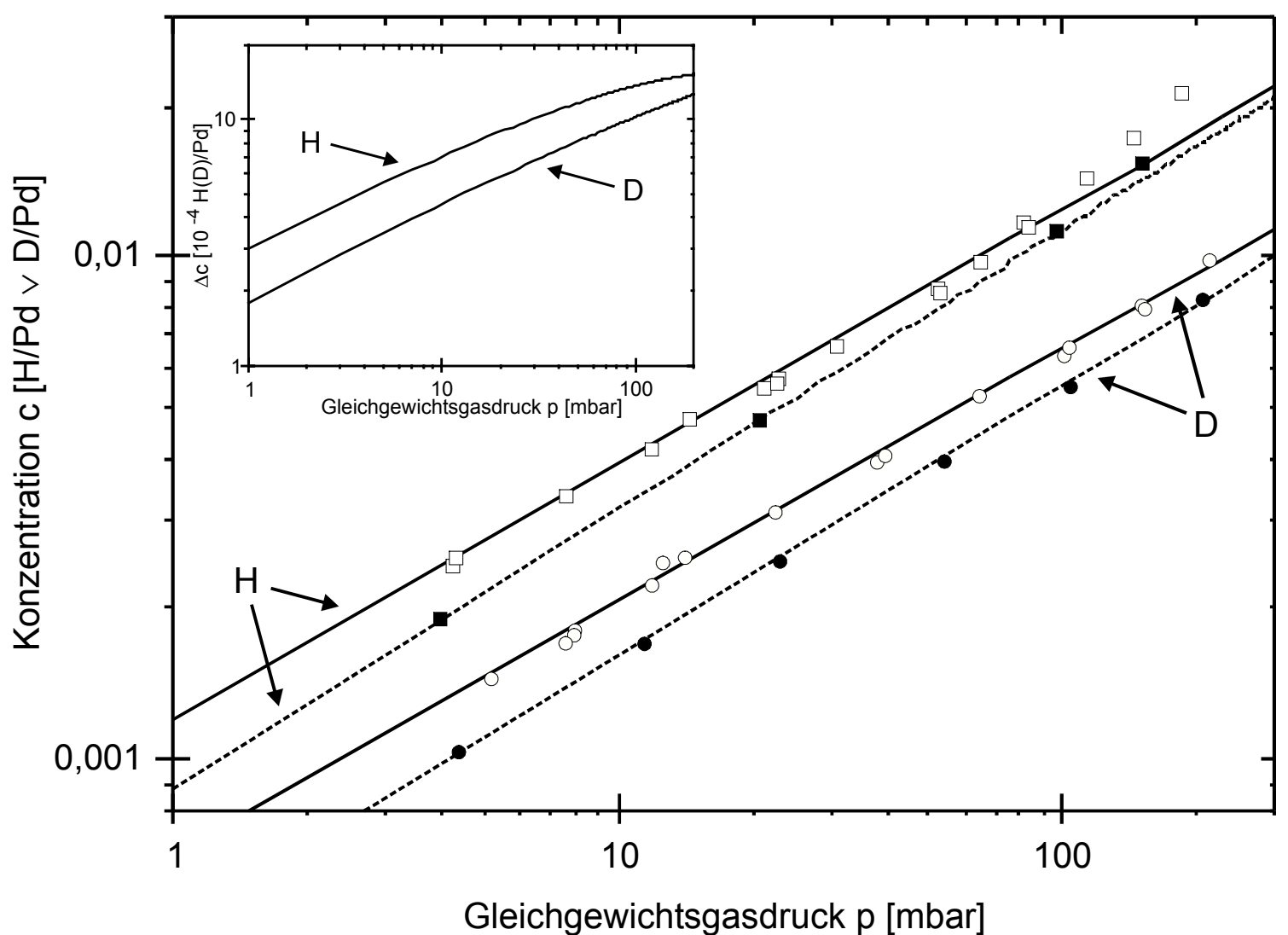

Abbildung 5.1: Doppeltlogarithmische Auftragung der Konzentrations-Druckisothermen für $80^{\circ} \mathrm{C}$. Die Sorptionsmessungen von Wasserstoff werden durch Quadrate symbolisiert, wobei sich geschlossene Quadrate $\mathbf{a u f}$ Messungen an geglühten Proben und offene Quadrate $\square$ auf Messungen an zyklierten Proben beziehen. Entsprechend werden geschlossene Kreise - Deuterium und geglühten Proben bzw. offene Kreise O Deuterium und zyklierten Proben zugeordnet. Die Kurven folgen aus der Anpassung von Gleichung 4.9 an die Messwerte. Das eingepasste Diagramm zeigt für Wasserstoff und Deuterium jeweils die Differenz $\Delta \mathrm{c}$ zwischen zyklierten und geglühten Proben. 
Eine Auftragung der Löslichkeitserhöhung $\Delta \mathrm{c}$ gegen $\mathrm{c}_{\mathrm{f}}$ eliminiert den in Kapitel 4.2.2 beschriebenen Isotopeneffekt zwischen Gasphase und interstitieller Lösung. Abbildung 5.2 zeigt die Zusammenhänge, die sich aus den Ergebnissen der angepassten Isothermen (Tabelle 4.4 ) ergeben. Die Übereinstimmung der Wasserstoff- und Deuteriumkurve ist im Rahmen der Messgenauigkeit bemerkenswert.

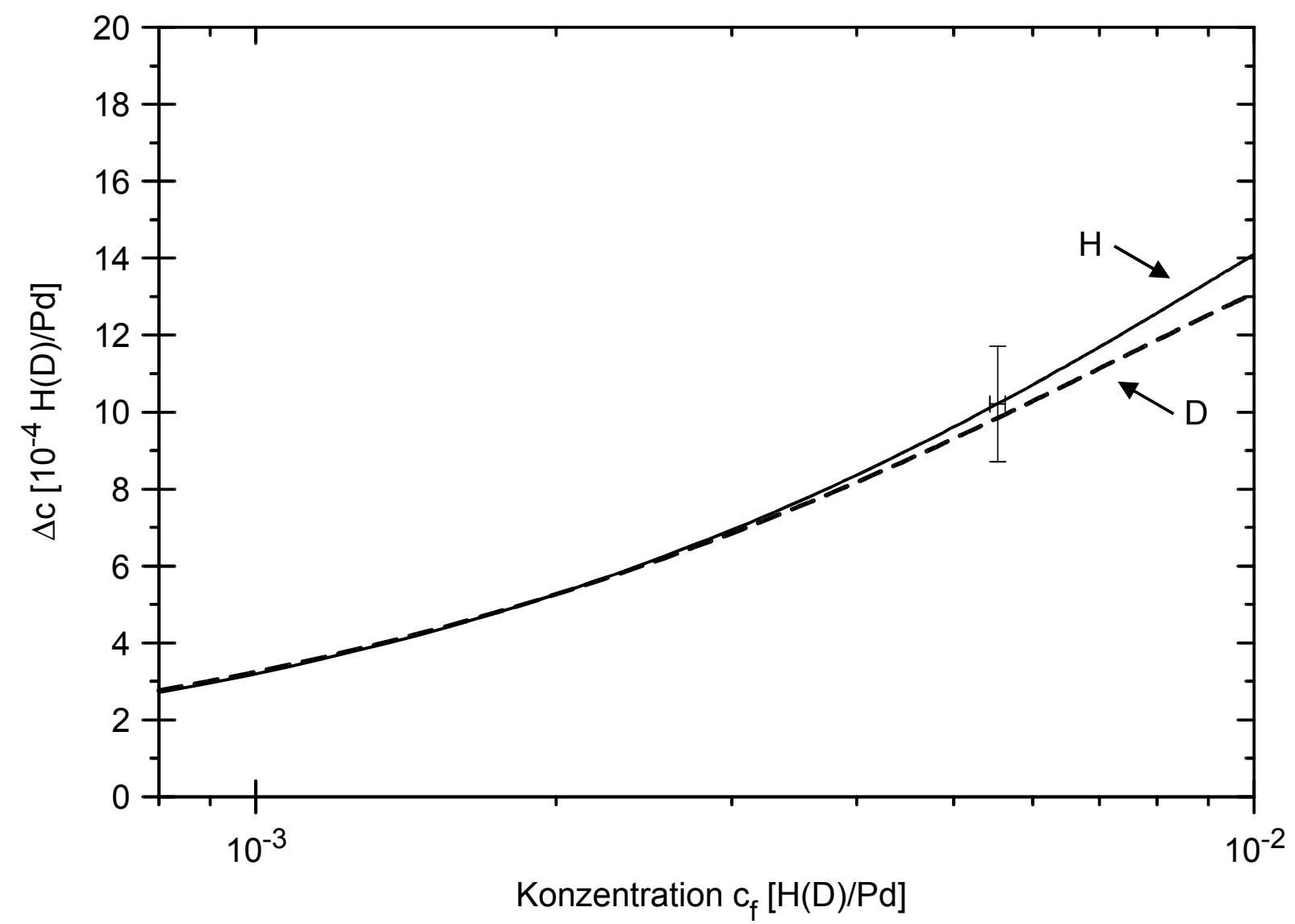

Abbildung 5.2: Halblogarithmische Auftragung der Löslichkeitserhöhung $\Delta \mathrm{c}$ einer zyklierten Probe gegen die Konzentration $c_{f}$ des „freien” Wasserstoffes (durchgezogene Linie) bzw. „freien” Deuteriums (gestrichelte Linie).

Damit erfolgt der experimentelle Nachweis, dass die Löslichkeitserhöhung $\Delta c$ durch die Segregation an Versetzungen für Wasserstoff und Deuterium in der $\alpha$-Phase gleich stark ist:

$$
\Delta \mathrm{c}_{\mathrm{H}}\left(\mathrm{c}_{\mathrm{f}}\right)=\Delta \mathrm{c}_{\mathrm{D}}\left(\mathrm{c}_{\mathrm{f}}\right)
$$

Dieses Ergebnis rechtfertigt die Annahme einer für Wasserstoff und Deuterium gleichen Wechselwirkung mit Versetzungen, wie sie den elastischen Argumenten von Gleichung 2.24 zugrunde liegt. 
Außerdem kann die Löslichkeitserhöhung für die gasvolumetrisch zyklierte Probe quantifiziert werden:

$$
\Delta \mathrm{c}=0,852 \cdot \mathrm{c}_{\mathrm{f}}^{0,937}-\mathrm{c}_{\mathrm{f}}
$$

$\mathrm{Zu}$ ihrer Bestimmung werden die Deuteriumdaten verwendet, da diese aufgrund einer größeren Anzahl an Messwerten eine geringere statistische Streuung aufweisen. Der Fehler in der Löslichkeitserhöhung wird mit $10 \%$ abgeschätzt. Einige Lösungen von Gleichung 5.4 sind in Tabelle 5.1 aufgeführt und können zudem Abbildung 5.2 entnommen werden. Die relative Löslichkeitserhöhung $\mathrm{c} / \mathrm{c}_{\mathrm{f}}$ liegt in der $\alpha$-Phase zwischen 1,3 und 1,15. Flanagan gibt für gewalztes Palladium einen Wert von $1,23 \pm 0,02$ bei $50{ }^{\circ} \mathrm{C}$ für $\mathrm{c} / \mathrm{c}_{\mathrm{f}}$ an, der unabhängig von der Konzentration ist [Fla76a]. Heuser findet bei Raumtemperatur und einer Konzentration $\mathrm{c}=5,5 \cdot 10^{-3} \mathrm{D} / \mathrm{Pd}$ für zykliertes Palladium Werte um 1,3 [Heu97]. Die hier bestimmte relative Löslichkeitserhöhung bei $80^{\circ} \mathrm{C}$ an einer gasvolumetrisch zyklierten Palladiumprobe ist wegen der höheren Temperatur und eventuell wegen einer kleineren Versetzungsdichte schächer ausgeprägt, und steht somit in guter Übereinstimmung mit den bekannten Werten.

Tabelle 5.1: Löslichkeitserhöhung einer gasvolumetrisch zyklierten Palladiumprobe für verschiedene Konzentrationen bei $80^{\circ} \mathrm{C}$.

\begin{tabular}{|cc|c|c|c|c|c|c|}
\hline $\mathrm{c}$ & {$\left[10^{-2} \mathrm{H} / \mathrm{Pd}\right]$} & 0,30 & 0,40 & 0,50 & 0,85 & 1,05 & 1,30 \\
\hline $\mathrm{c}_{\mathrm{f}}$ & {$\left[10^{-2} \mathrm{H} / \mathrm{Pd}\right]$} & 0,24 & 0,327 & 0,415 & 0,73 & 0,915 & 1,15 \\
\hline$\Delta \mathrm{c}$ & {$\left[10^{-2} \mathrm{H} / \mathrm{Pd}\right]$} & 0,06 & 0,073 & 0,085 & 0,12 & 0,135 & 0,15 \\
\hline $\mathrm{c} / \mathrm{c}_{\mathrm{f}}$ & & 1,25 & 1,22 & 1,20 & 1,16 & 1,15 & 1,13 \\
\hline
\end{tabular}

\subsection{Ergebnisse der Kleinwinkelneutronenstreuung}

In der Kleinwinkelstreuung bestimmt die Streulängendichtedifferenz $\Delta \rho$ und die Geometrie des streuenden Teilchens den kohärenten Netto-Streuquerschnitt. Dies ermöglicht es, die Segregation von Wasserstoff an Versetzungen zu charakterisieren. Für die radiale Ausdehnung des Segregationsgebietes kann eine Abhängigkeit von der Wasserstoffkonzentration in der $\alpha$-Phase nachgewiesen werden, die in Kapitel 5.2.1 diskutiert wird. Für deuterierte Proben ist der Kontrast weniger stark ausgeprägt und wird in Kapitel 5.2.2 interpretiert. Eine entsprechende Kontrastvariation wird beim Porodverhalten (Kapitel 5.2.3) beobachtet.

Im Folgenden bezeichnet $\mathrm{d} \Sigma / \mathrm{d} \Omega$ grundsätzlich den kohärenten Netto-Streuquerschnitt. 


\subsubsection{Zylinderradius in Abhängigkeit von der Wasserstoffkonzentration}

In diesem Kapitel wird die systematische Untersuchung des Streuverhaltens eines Palladiumwalzbleches in Abhängigkeit von der Wasserstoffkonzentration c in der $\alpha$-Phase vorgestellt. Die Versetzungsdichte $\zeta$ der Probe wurde, wie in Kapitel 4.1.2 beschrieben, durch elektrochemisches Zyklieren bei Raumtemperatur erhöht und elektronenmikroskopisch zu $5 \cdot 10^{11} \mathrm{~cm}^{-2}$ bestimmt (Kapitel 4.1.3).

Abbildung 4.10 zeigt die Streukurve der unbeladenen Probe und exemplarisch eine Streukurve des beladenen Zustandes, die um den inkohärenten Streubeitrag des gelösten Wasserstoffes vermindert wurde. Der vom Streuvektor unabhängige inkohärente Streuquerschnitt bestimmt sich gemäß Gleichung 4.13 mit den in Tabelle 4.6 dargestellten Werten. Der NettoStreuquerschnitt $\mathrm{d} \Sigma / \mathrm{d} \Omega$ wird als Differenz der Streuquerschnitte der Probe im beladenen und unbeladenen Zustand entsprechend Gleichung 4.12 berechnet. Den kohärenten Netto-Streuquerschnitt zeigt Abbildung 5.3 in doppeltlogarithmischer Auftragung.

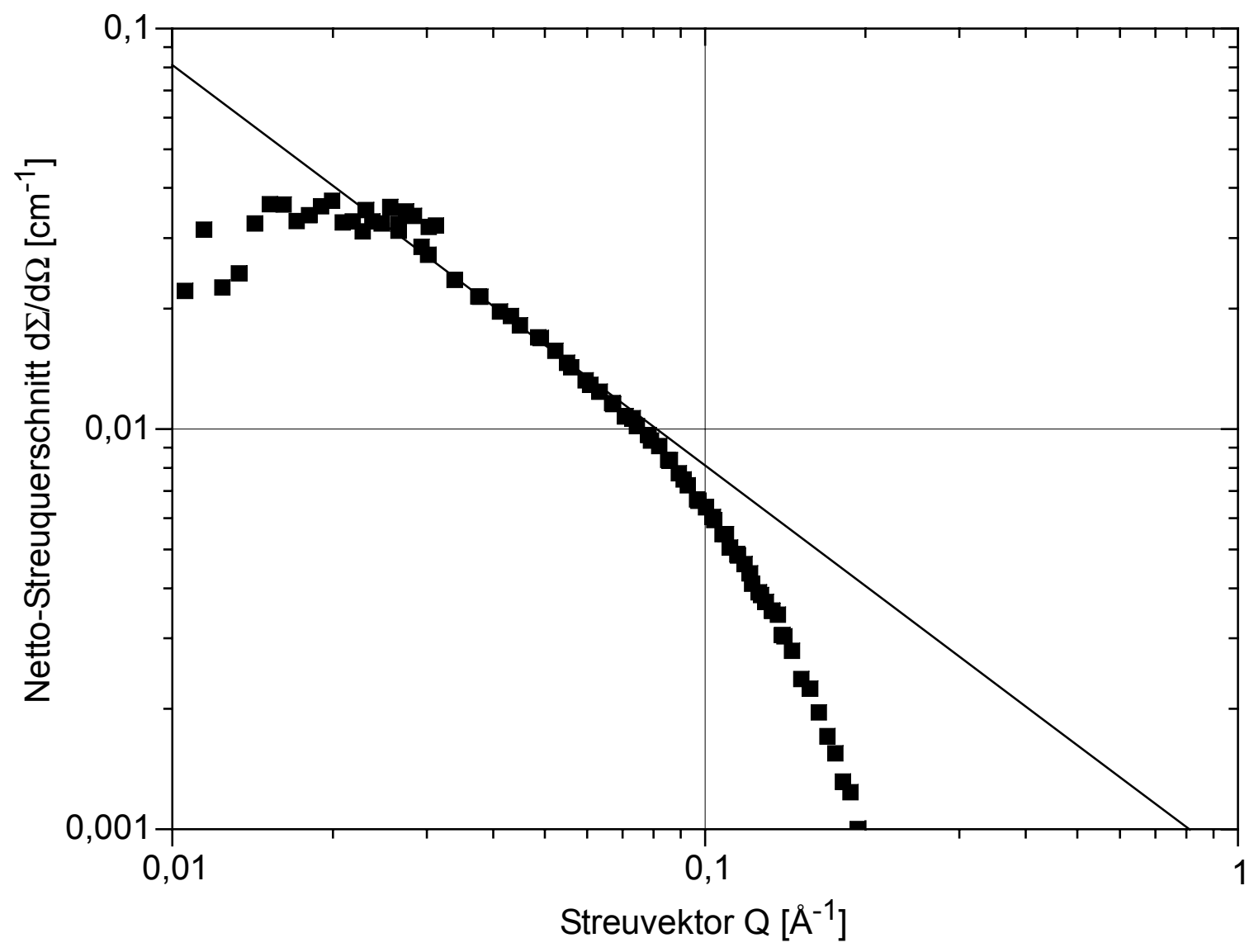

Abbildung 5.3: Doppeltlogarithmische Auftragung des kohärenten Netto-Streuquerschnittes einer versetzungsreichen Palladiumprobe beladen mit 0,0105 H/Pd. Die Gerade entspricht $\mathrm{d} \Sigma / \mathrm{d} \Omega \propto \mathrm{Q}^{-1}$. 
An den kohäherenten Netto-Streuquerschnitt kann für mittlere Werte des Streuvektors Q eine $\mathrm{Q}^{-1}$-Kurve angepasst werden, die in Abbildung 5.3 dargestellt ist. Ein $\mathrm{Q}^{-1}$-Verhalten wird für linienhafte Streuobjekte erwartet, deren Breite $2 \mathrm{R}$ klein gegen ihre Länge $2 \mathrm{H}$ ist, wobei $\mathrm{HQ} \gg 1$ und $\mathrm{RQ} \ll 1$ gelten muss. Demnach scheinen die mit Wasserstoff angereicherten Gebiete entlang der Versetzungslinie diese Bedingung zu erfüllen. Für die so dekorierte Versetzung beschreibt Gleichung 3.33 den Netto-Streuquerschnitt einer Probe mit der Versetzungsdichte $\zeta$. Es handelt sich um eine eindimensionale Modellierung.

$$
\frac{\mathrm{d} \Sigma}{\mathrm{d} \Omega}=\pi \cdot \zeta \cdot\left(\pi \mathrm{R}^{2}\right)^{2} \cdot \frac{\Delta \rho^{2}}{\mathrm{Q}}
$$

Abbildung 5.3 zeigt ein Abweichen vom $\mathrm{Q}^{-1}$-Verhalten sowohl bei kleinen als auch bei größeren Werten des Streuvektors Q. Der Effekt bei kleinen Q-Werten ist auf das durch die Beladung modifizierte Porodverhalten zurückzuführen, welches in Kapitel 5.2.3 diskutiert wird. Die Abweichung des Netto-Streuquerschnittes für größere Q-Werte deutet darauf hin, dass die Gleichung 5.5 zugrunde liegende Annahme eines linienhaften Streuobjektes um eine radiale Dimension zu erweitern ist. Dies führt zu der zylinderförmigen Beschreibung des Segregationsgebietes aus Kapitel 2.4. Das Streuverhalten stochastisch orientierter Zylinder mit Radius R und einer Streulängendichtedifferenz $\Delta \rho$ zur Matrix in einer Probe der Versetzungsdichte $\zeta$ folgt Gleichung 3.32:

$$
\frac{\mathrm{d} \Sigma}{\mathrm{d} \Omega}=\frac{\pi \cdot \zeta \cdot\left(\pi \mathrm{R}^{2}\right)^{2} \cdot \Delta \rho^{2}}{\mathrm{Q}} \cdot \exp \left(-\frac{\mathrm{Q}^{2} \mathrm{R}^{2}}{4}\right)
$$

Der Exponentialterm berücksichtigt die Abweichung vom eindimensionalen Verhalten.

Die Auftragung von $\ln \left(\mathrm{Q} \cdot \frac{\mathrm{d} \Sigma}{\mathrm{d} \Omega}\right)$ gegen $\mathrm{Q}^{2}$ wird im Folgenden als modifizierter Guinierplot bezeichnet. Abbildung 5.4 zeigt exemplarisch den modifizierten Guinierplot, der sich aus dem kohärenten Netto-Streuquerschnitt von Abbildung 5.3 ergibt. Die Messwerte folgen über einen weiten $\mathrm{Q}^{2}$-Bereich dem gemäß Gleichung 5.6 erwarteten linearen Verhalten, dessen Geradensteigung $-\mathrm{R}^{2} / 4$ entspricht. Der modifizierte Guinierplot wird auf diese Weise zur Bestimmung des Zylinderradius verwendet. Die Übereinstimmung der experimentell bestimmten Streuquerschnitte mit Gleichung 5.6 ist ausgeprägt und rechtfertigt somit die Annahme des zylinderförmigen Segregationsgebietes mit scharfer Grenzfläche, die sowohl in Kapitel 3.3 als auch in Kapitel 2.4 verwendet wurde. Der steile Abfall der Messwerte bei kleinen $Q^{2}$-Werten in Abbildung 5.4 wird wiederum dem Porodbeitrag zugeschrieben. 


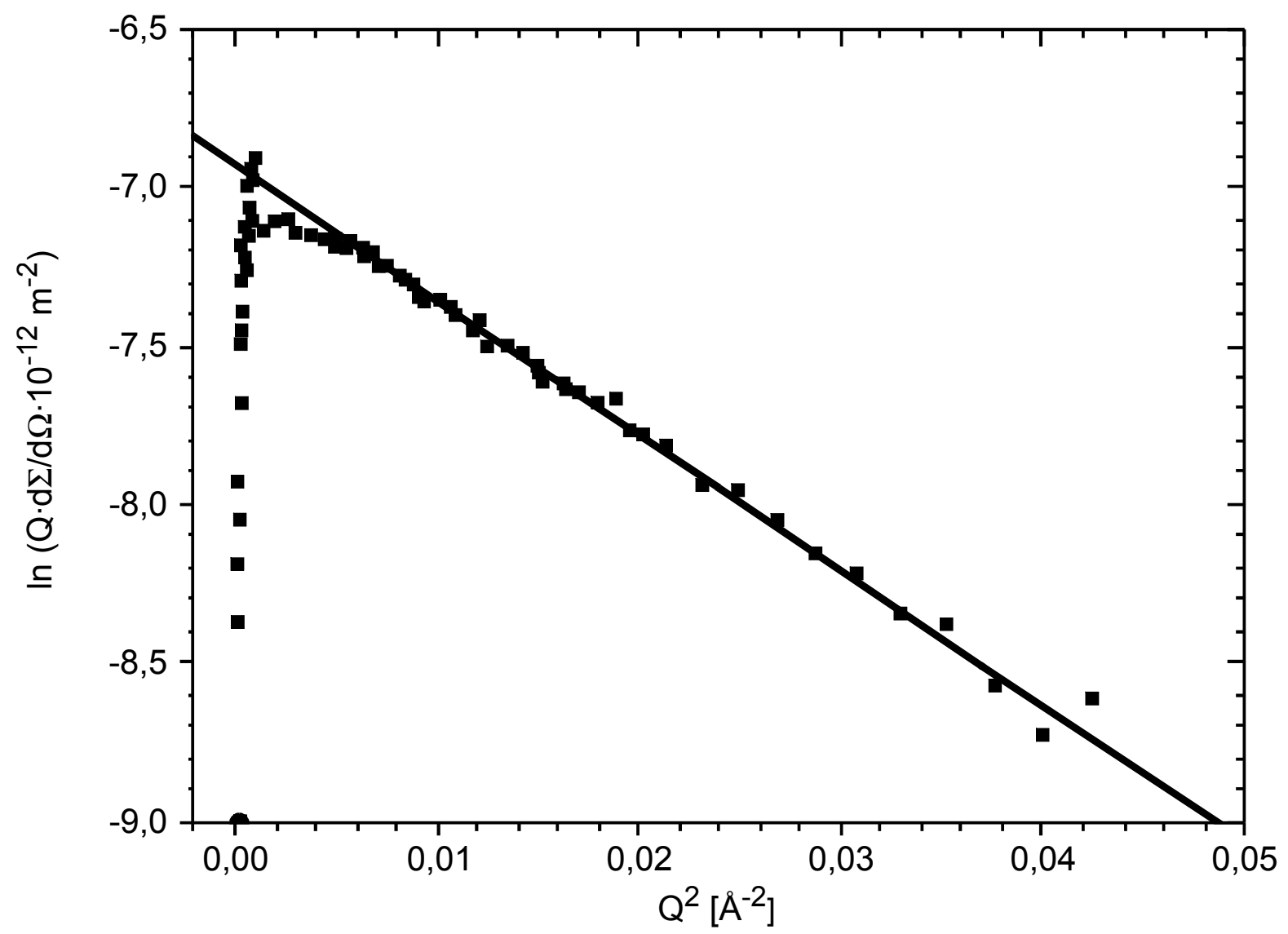

Abbildung 5.4: Modifizierter Guinierplot zu einer Beladung von 0,0105 H/Pd. Die Steigung der an die Messwerte angepassten Geraden entspricht $-\mathrm{R}^{2} / 4$.

Zentrale Aufgabe dieser Arbeit ist die systematische Untersuchung der Abhängigkeit des Zylinderradius R von der Wasserstoffkonzentration $\mathrm{c}_{\mathrm{H}}$. Dazu werden Streukurven der gewalzten und dann zyklierten Probe aufgenommen, die elektrochemisch mit definierten Wasserstoffkonzentrationen beladen sind. Abbildung 5.5 zeigt eine Übersicht aller entsprechenden NettoStreuquerschnitte. Sie verlaufen ähnlich wie die in Abbildung 5.3 dargestellte und bereits diskutierte Kurve. Abweichungen werden lediglich bei den Messungen zu einer Wasserstoffkonzentration von $1,3 \cdot 10^{-2} \mathrm{H} / \mathrm{Pd}$ und $1,7 \cdot 10^{-2} \mathrm{H} / \mathrm{Pd}$ beobachtet. Da beide Konzentrationen oberhalb der maximalen Löslichkeit der $\alpha$-Phase bei Raumtemperatur liegen, ist anzunehmen, dass Ausscheidungen der $\beta$-Phase für das abweichende Verhalten verantwortlich sind. Die Netto-Streuquerschnitte für höhere Konzentrationen besitzen aufgrund des mit der Wasserstoffkonzentration zunehmenden Streubeitrages der Segregationszylinder eine geringere statistische Streuung.

Die oben beschriebene Auswertung im modifizierten Guinierplot wurde für die verschiedenen Konzentrationen durchgeführt. Wie unmittelbar in Abbildung 5.4 ersichtlich ist, können die Messwerte durch einen linearen Zusammenhang beschrieben werden. Das veränderte Streuverhalten bei Konzentrationen oberhalb der maximalen Löslichkeit von $\alpha$-Palladium führt bei $1,3 \cdot 10^{-2} \mathrm{H} / \mathrm{Pd}$ zu einer erhöhten jedoch noch tolerierbaren Unsicherheit. Bei $1,7 \cdot 10^{-2} \mathrm{H} / \mathrm{Pd}$ ist 
die Übersättigung derart groß, dass die resultierende Krümmung im modifizierten Guinierplot keine Auswertung zulässt. Die gemäß Gleichung 5.6 bestimmten Zylinderradien sind in Tabelle 5.2 aufgeführt. Sie steigen erwartungsgemäß innerhalb der $\alpha$-Phase mit der Wasserstoffkonzentration an. Außerhalb der $\alpha$-Phase setzt sich dieser Trend nicht fort, so dass bei der Konzentration von 1,3·10 ${ }^{-2} \mathrm{H} / \mathrm{Pd}$ ein tendenziell kleinerer Zylinderradius gemessen wurde.

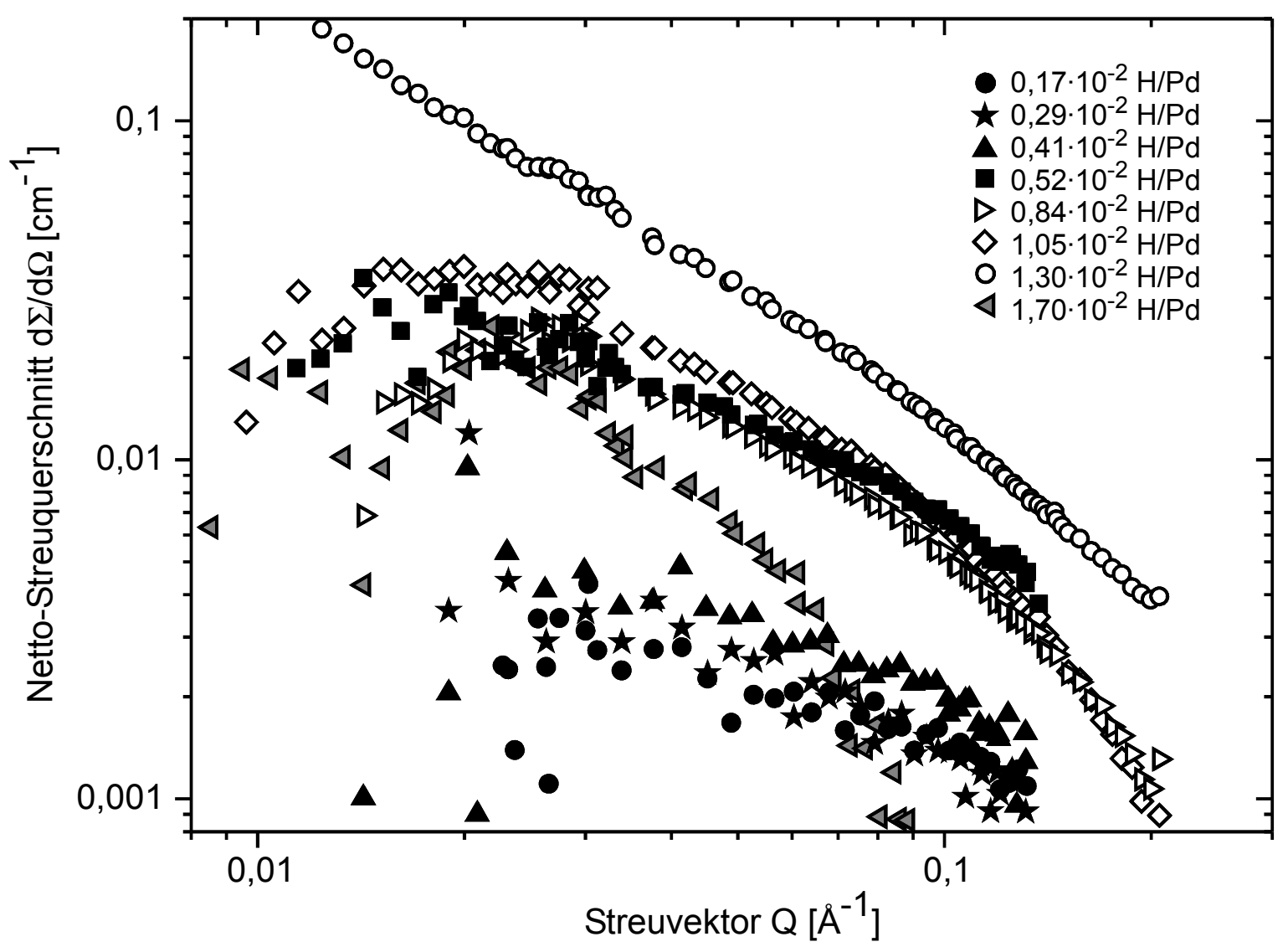

Abbildung 5.5: Übersicht der Netto-Streuquerschnitte in doppeltlogarithmischer Darstellung für die gewalzte und dann zyklierte Probe mit den angegebenen Wasserstoffkonzentrationen. Elektrochemische Beladung und Messung erfolgten bei Raumtemperatur.

Tabelle 5.2: $\quad$ Übersicht der aus Streuexperimenten bestimmten Zylinderradien $R$ in Abhängigkeit von der Wasserstoffkonzentration $\mathrm{c}_{\mathrm{H}}$

\begin{tabular}{|c|c|c|c|c|c|c|c|}
\hline $\mathrm{c}_{\mathrm{H}}\left[10^{-2} \mathrm{H} / \mathrm{Pd}\right]$ & 0,17 & 0,29 & 0,41 & 0,52 & 0,84 & 1,05 & 1,3 \\
\hline $\mathrm{R}[\AA]$ & 4,9 & 5,1 & 6,3 & 8,3 & 10,8 & 13,1 & 12,0 \\
\hline
\end{tabular}

Die im Rahmen dieser Arbeit gemessenen Radien des zylinderförmigen Segregationsgebietes von Wasserstoff an Stufenversetzungen liegen im Bereich 5 ... $13 \AA$. Heuser und King ermitteln an zyklierten Palladiumeinkristallen bei einer Deuteriumkonzentration von 
$0,55 \cdot 10^{-2} \mathrm{D} / \mathrm{Pd}$ ebenfalls Zylinderradien von $7 \ldots 11 \AA$ mittels Kleinwinkelneutronenstreuung [Heu97]. Theoretische Rechnungen von Ross und Stefanopoulos zeigen hingegen einen Abfall der Wasserstoffkonzentration von ihrem Maximalwert im Segregationsgebiet bei einer radialen Ausdehnung von $3 \ldots 5 \AA$ [Ros94]. Die Autoren nehmen lediglich eine rein elastische Wechselwirkung von Wasserstoff mit dem Stufenanteil einer Versetzung an. Die experimentellen Ergebnisse legen jedoch nahe, dass eine zusätzliche attraktive Wechselwirkung zu berücksichtigen ist, die zu einer Vergrößerung des Segregationsgebietes führt. In Kapitel 2.4 wurde ein thermodynamisches Modell vorgestellt, das neben dem elastischen Beitrag (Gleichung 2.24) auch eine konstante chemische Wechselwirkung analog zur Hydridbildung berücksichtigt. Abbildung 5.6 zeigt eine Anpassung dieses Modells an die gemessenen Zylinderradien, deren Ergebnis im Folgenden diskutiert wird.

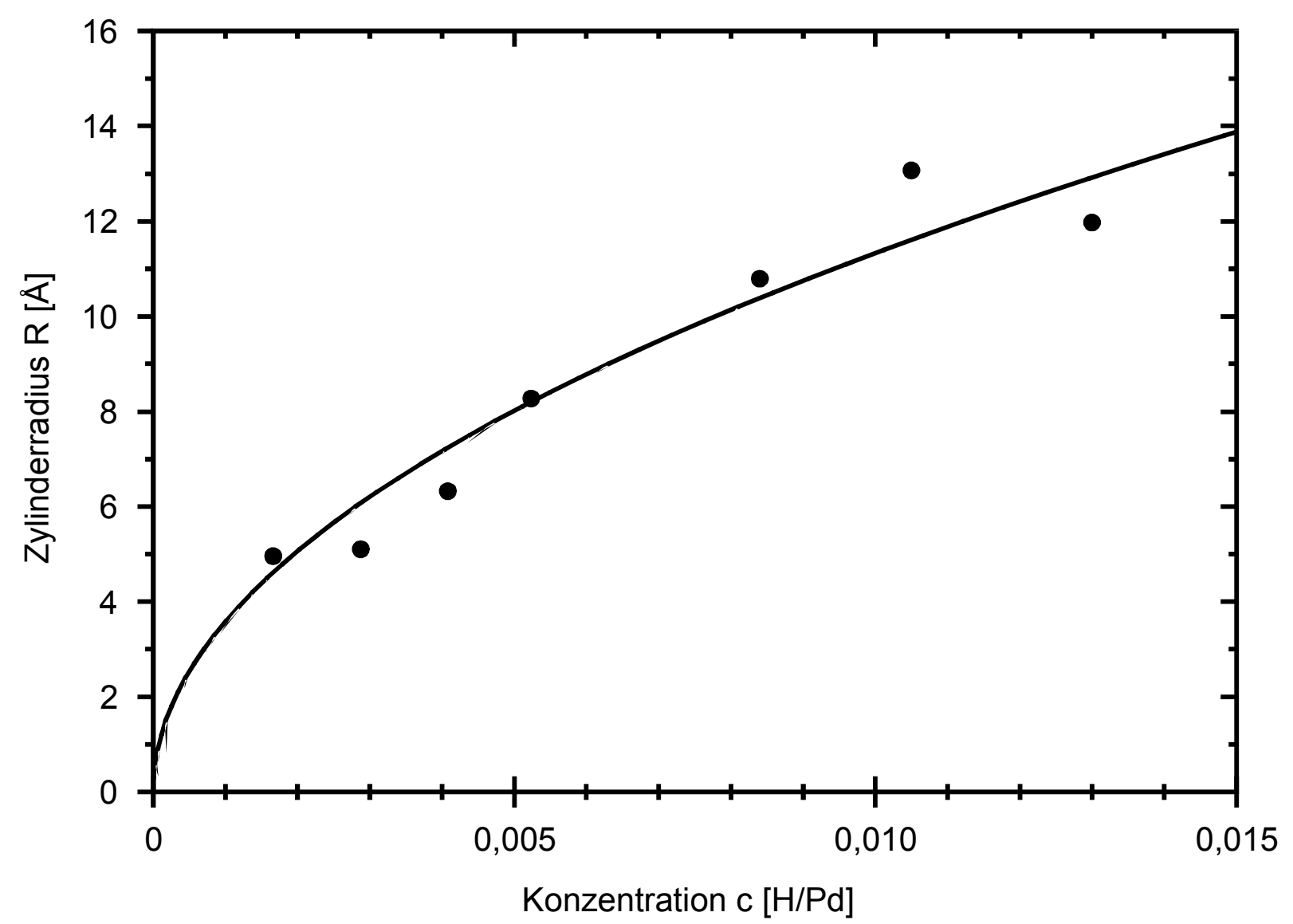

Abbildung 5.6: Abhängigkeit der Zylinderradien von der Wasserstoffkonzentration. Die Kurve stellt eine Anpassung von Gleichung 5.7 an die Messwerte dar. 
Die Abhängigkeit der Zylinderradien von der Wasserstoffkonzentration ist mit den Daten aus Tabelle 5.2 in Abbildung 5.6 dargestellt. An die Ergebnisse der Neutronenstreuung wurde Gleichung 2.31 angepasst, die das bereits erwähnte thermodynamische Modell aus Kapitel 2.4 beschreibt:

$$
\mathrm{c}=\mathrm{c}_{\mathrm{ss}} \cdot \zeta \pi \mathrm{R}^{2}+\mathrm{c}_{\mathrm{s}}^{0} \cdot \exp \left(-\frac{\mathrm{A}}{\mathrm{R}}\right)
$$

Dabei geht die maximale Löslichkeit $\mathrm{c}_{\mathrm{s}}^{0}$ der $\alpha$-Phase, die Besetzung $\mathrm{c}_{\mathrm{ss}}$ der Zwischengitterplätze im Segregationszylinder und die in Gleichung 2.30 definierte Konstante A ein. Letztere folgt aus dem Elastizitätsmodul von Palladium, der entsprechenden Poissonschen Querkontraktionszahl, dem Burgersvektor der Stufenversetzung und dem partiellen Atomvolumen von Wasserstoff in Metallen. Aus der Anpassung wurde eine Versetzungsdichte $\zeta$ von $(2,4 \pm 0,3) \cdot 10^{11} \mathrm{~cm}^{-2}$ bestimmt. Die Übereinstimmung $\mathrm{zu}$ der in Kapitel 4.1.3 elektronenmikroskopisch ermittelten Versetzungsdichte von $5 \cdot 10^{11} \mathrm{~cm}^{-2}$ ist insbesondere unter Berücksichtigung der dort verwendeten robusten Schätzung ausgezeichnet.

Wie in Abbildung 5.6 ersichtlich ist, werden die gemessenen Zylinderradien durch das in Kapitel 2.4 vorgestellte einfache thermodynamische Modell gut beschrieben. Die erfolgreiche Verknüpfung der Zylinderradien aus Neutronenkleinwinkelstreumessungen über die Konstante A mit den oben genannten Materialkonstanten von Palladium und der Versetzungsdichte aus elektronenmikroskopischen Untersuchungen rechtfertigt die Berücksichtigung einer direkten HH-Wechselwirkung zusätzlich zur elastischen Wechselwirkung des Wasserstoffes mit Versetzungen.

Die hier beschriebene Interpretation der Ergebnisse aus Neutronenstreuexperimenten kann auf ihre Konsistenz überprüft werden, indem die Versetzungsdichte zusätzlich direkt aus den Streukurven bestimmt wird. Die Achsenabschnitte der Anpassungen des $\mathrm{Q}^{-1}$-Verhaltens gemäß Gleichung 5.5 an die Netto-Streuquerschnitte (Abbildung 5.3) und von Gleichung 5.6 im modifizierten Guinierplot (Abbildung 5.4) ergeben beide eine Relation für $\zeta \cdot R^{4} \cdot \Delta \rho^{2}$. Die darin eingehende Streulängendichtedifferenz $\Delta \rho=\rho_{\text {Hydrid }}-\rho_{\text {Pd }}$ kann aus den folgenden Abhängigkeiten berechnet werden:

$$
\begin{aligned}
\rho_{\mathrm{Pd}} & =\mathrm{N}_{\mathrm{A}} \cdot \frac{\mathrm{b}_{\mathrm{Pd}}}{\mathrm{V}_{\mathrm{Pd}}}=4,02 \cdot 10^{10} \mathrm{~cm}^{-2} \\
\rho_{\text {Hydrid }} & =\mathrm{N}_{\mathrm{A}} \cdot \frac{\mathrm{b}_{\mathrm{Pd}}+\mathrm{c}_{\mathrm{ss}} \cdot \mathrm{b}_{\mathrm{H}}}{\mathrm{V}_{\mathrm{Pd}}+\mathrm{f} \cdot \mathrm{c}_{\mathrm{ss}} \cdot \overline{\mathrm{V}}} \underset{\mathrm{f}=1}{=} 2,23 \cdot 10^{10} \mathrm{~cm}^{-2}
\end{aligned}
$$

Dabei gehen die Streulängen $b_{P d}$ und $b_{H}$ von Palladium und Wasserstoff, das Molvolumen $V_{P d}$ von Palladium, das partielle Molvolumen $\overline{\mathrm{V}}$ von Wasserstoff in Metallen, die Besetzung $\mathrm{c}_{\mathrm{ss}}$ der Zwischengitterplätze im Segregationszylinder und der Faktor f ein, der den Grad der Aus- 
dehnung des Segregationszylinders in der Palladiummatrix beschreibt. Eine vollständige Ausdehnung wird durch $f=1$ ausgedrückt. Die Streulängendichtedifferenz ist von dem Faktor $f$ abhängig:

$$
\Delta \rho=N_{A} \cdot c_{s s} \cdot \frac{b_{H} \cdot V_{P d}-f \cdot b_{P d} \cdot \bar{V}}{\left(V_{P d}+f \cdot c_{s s} \cdot \bar{V}\right) \cdot V_{P d}}
$$

Bei vollständiger Ausdehnung $(\mathrm{f}=1)$ und einer Konzentration $\mathrm{c}_{\mathrm{ss}}=0,6$ im Segregationszylinder besitzt die Streulängendichtedifferenz $\Delta \rho_{\mathrm{H}}$ den Wert $-1,79 \cdot 10^{10} \mathrm{~cm}^{-2}$. Aus den Achsenabschnitten der oben beschriebenen Anpassungen kann mit diesem Wert und den Radien aus Tabelle 5.2 die Versetzungsdichte $\zeta$ direkt aus den Streukurven bestimmt werden. Abbildung 5.7 zeigt die Ergebnisse, die alle dieselbe Größenordnung besitzen wie die elektronenmikroskopisch ermittelte Versetzungsdichte von $5 \cdot 10^{11} \mathrm{~cm}^{-2}$.

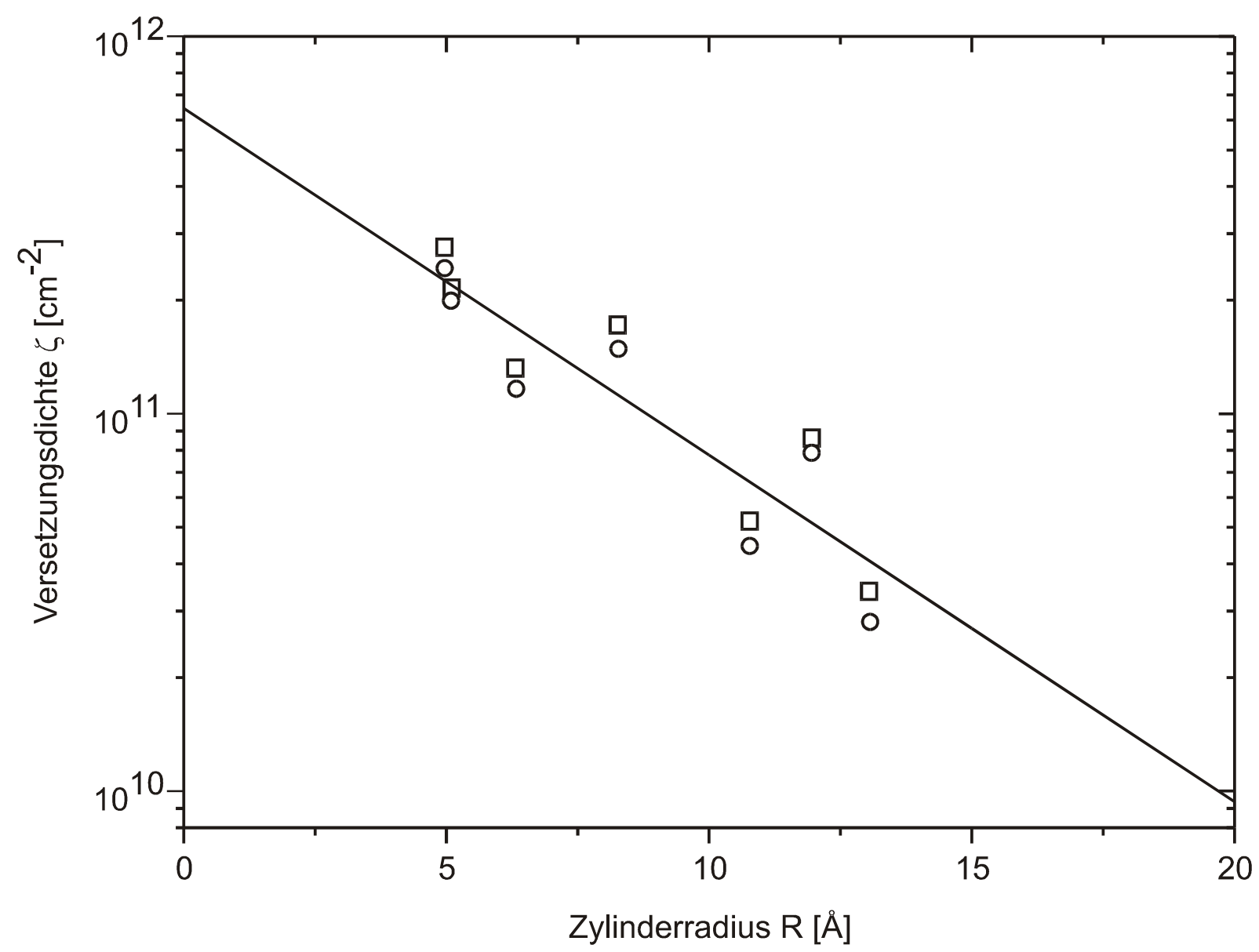

Abbildung 5.7: Auftragung der Versetzungsdichte gegen den Zylinderradius. Die Versetzungsdichte wurde jeweils aus den Achsenabschnitten der $\mathrm{Q}^{-1}$-Anpassung des Nettostreuquerschnittes (O) und der Anpassung im modifizierten Guinierplot $(\square)$ bestimmt. Die Gerade wurde durch Anpassung an alle Werte erhalten. 
Die Versetzungsdichte in Abbildung 5.7 scheint neben der Übereinstimmung in der Größenordnung außerdem mit steigendem Zylinderradius abzunehmen. Die Anpassung einer Gerade an die Messwerte ergibt eine Versetzungsdichte von $6 \cdot 10^{11} \mathrm{~cm}^{-2}$ bei $\mathrm{R}=0$ und damit eine gute Übereinstimmung mit dem Ergebnis der Transmissionselektronenmikroskopie. Die abnehmende Versetzungsdichte kann möglicherweise auf zwei Effekte zurückgeführt werden. Zum einen führt die elektrochemische Beladung zu einem Konzentrationsgradienten in der Probe, der mit großen Spannungen verbunden ist. Dadurch können Defekte und somit auch Versetzungen ausheilen. Schaefer konnte beispielsweise durch Positronenlebenszeitspektroskopie nachweisen, dass eine Einzelleerstelle in einkristallinem Palladium bereits bei $389 \mathrm{~K}$ mobil ist [Sch82]. In defektreichen Materialien ist diese Temperatur üblicherweise reduziert. In diesem Fall wäre auch von einer Abnahme der Versetzungsdichte auf langer Zeitskala auszugehen. Der Vergleich von Streukurven, die mit langem zeitlichen Abstand zueinander aufgenommen wurden, scheint diese Annahme zu bestätigen.

Ein Überschätzen des Zylinderradius würde ebenfalls zu einer Abnahme der Versetzungsdichte führen. Dies könnte durch einen Streubeitrag, der dem Beitrag der Segregationszylinder überlagert ist, erklärt werden. Mit Wasserstoff dekorierte Leerstellencluster oder Zellwände von Versetzungen würden einen entsprechenden Kontrast liefern. Da der Radius in die oben vorgestellte Bestimmung der Versetzungsdichte mit der vierten Potenz eingeht, könnten bereits kleine Beiträge den Effekt erklären.

Die experimentellen Ergebnisse dieser Arbeit widersprechen keiner der beiden Erklärungen.

Eine weitere Möglichkeit, das thermodynamische Modell zu überprüfen, bietet der Vergleich der gasvolumetrisch bestimmten Löslichkeitserhöhung $\Delta \mathrm{c}$ mit der Wasserstoffmenge, die an den Versetzungen gebunden ist. In Tabelle 5.3 sind die Werte für die einzelnen Zylinderradien dargestellt, die sich gemäß $\Delta \mathrm{c}=\mathrm{c}_{\mathrm{ss}} \cdot \zeta \cdot \pi \mathrm{R}^{2}$ (Gleichung 5.7) ergeben. Die Ergebnisse der Gasvolumetrie bei den zugehörigen Wasserstoffkonzentrationen werden Tabelle 5.1 entnommen. Sie sind grundsätzlich kleiner als die Vorhersagen des Zylindermodells. Diese Tendenz kann durch die höhere Temperatur bei den gasvolumetrischen Messungen und die wahrscheinlich kleinere Versetzungsdichte der verwendeten Probe erklärt werden. Die Übereinstimmung der unabhängig experimentell gewonnenen Werte ist besser als eine Größenordnung und daher eine Bestätigung des Modells. Die Abweichnung nimmt allerdings mit dem Zylinderradius zu, was wiederum auf eine leichte Überschätzung des Zylinderradius hindeuten könnte. Entsprechende Überlegungen werden in Kapitel 5.2.2 diskutiert. 
Tabelle 5.3: Vergleich der an den Versetzungen gebundenen Wasserstoffmenge aus gasvolumetrischen Messungen und Streuexperimenten.

\begin{tabular}{|cc|c|c|c|c|c|c|}
\hline \multicolumn{7}{|c|}{ Löslichkeitserhöhung aus gasvolumetrischen Messungen } \\
\hline $\mathrm{c}$ & {$\left[10^{-2} \mathrm{H} / \mathrm{Pd}\right]$} & 0,30 & 0,40 & 0,50 & 0,85 & 1,05 & 1,30 \\
\hline$\Delta \mathrm{c}$ & {$\left[10^{-2} \mathrm{H} / \mathrm{Pd}\right]$} & 0,06 & 0,073 & 0,085 & 0,12 & 0,135 & 0,15 \\
\hline \multicolumn{7}{|c|}{ Löslichkeitserhöhung im Zylindermodell } \\
\hline $\mathrm{R}$ & {$[\AA]$} & 5,1 & 6,3 & 8,3 & 10,8 & 13,1 & 12,0 \\
\hline$\Delta \mathrm{c}$ & {$\left[10^{-2} \mathrm{H} / \mathrm{Pd}\right]$} & 0,12 & 0,18 & 0,38 & 0,53 & 0,78 & 0,65 \\
\hline
\end{tabular}

\subsubsection{Streuverhalten deuterierter Proben}

Das Streuverhalten der geglühten und dann zyklierten Probe wird im Folgenden in Abhängigkeit von der Deuteriumkonzentration dargestellt und im Vergleich zu den bei Wasserstoffbeladung erhaltenen Ergebnissen diskutiert. Außerdem ermöglicht das umgekehrte Vorzeichen der Streulänge $b_{D}$ von Deuterium in Bezug auf die Streulänge $b_{H}$ von Wasserstoff eine Kontrastvariation bei den Streuexperimenten.

Mit dieser Kontrastvariation kann die Ausdehnung des Segregationszylinders innerhalb der Palladiummatrix auf unabhängige Weise bestimmt werden.

Dazu wurden von Kirchheim [Kir98] zwei Messungen der Kleinwinkelneutronenstreuung einer geglühten und zyklierten Probe mit identischer Deuterium- bzw. Wasserstoffkonzentration von $0,8 \cdot 10^{-2}-\mathrm{H}(\mathrm{D}) / \mathrm{Pd}$ am NIST in Washington durchgeführt. Die Messungen und die gasvolumetrische Beladung der Proben erfolgte bei $80{ }^{\circ} \mathrm{C}$. Abbildung 5.8 zeigt die NettoStreuquerschnitte. Die Streukurven folgen sowohl bei der Beladung mit Wasserstoff als auch bei der Beladung mit Deuterium demselben $\mathrm{Q}^{-1}$-Verhalten, welches bezüglich der Abbildung 5.3 diskutiert wurde. Deuterium bildet scheinbar identisch zu Wasserstoff linienhafte Segregationsgebiete.

Da die gasvolumetrische Untersuchung der Löslichkeitserhöhung durch Versetzungen (Gleichung 5.3) ein identisches Segregationsverhalten von Wasserstoff und Deuterium (Abbildung 5.2) zeigt, kann von einer gleich starken attraktiven HH- bzw. DD-Wechselwirkung ausgegangen werden und die Segregationsgebiete müssen für beide Isotope dieselbe Dimension besitzen. Folglich kann die Segregation von Deuterium an Versetzungen analog zu der von Wasserstoff beschrieben werden. 


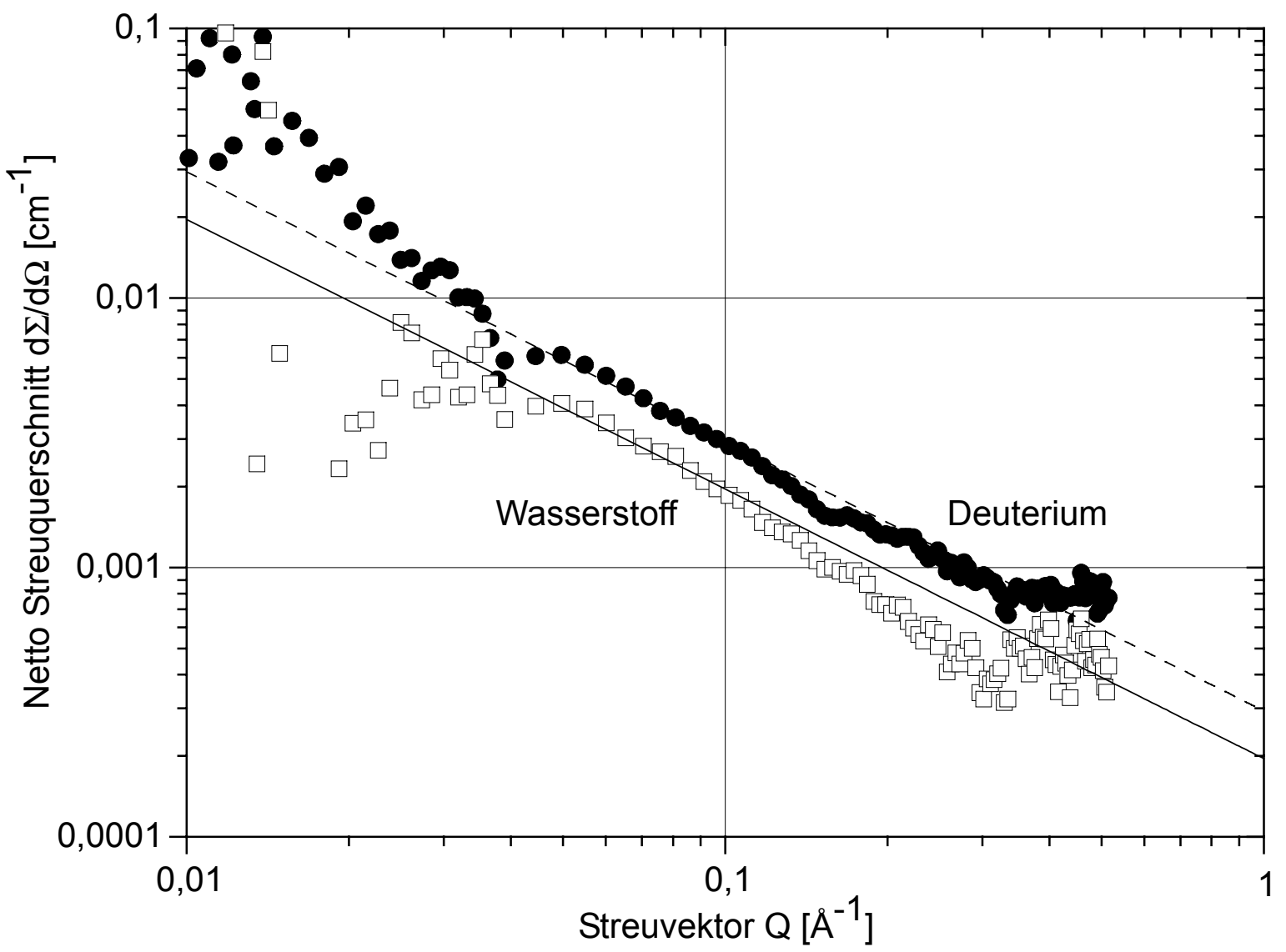

Abbildung 5.8: Vergleich des Streuverhaltens von Deuterium $(\bullet)$ und Wasserstoff $(\square)$ an einer identischen Probe, die bei $80^{\circ} \mathrm{C}$ gasvolumetrisch mit $0,008 \mathrm{H}(\mathrm{D}) / \mathrm{Pd}$ beladen wurde. Die eingezeichneten Kurven entsprechen $\mathrm{d} \Sigma / \mathrm{d} \Omega \propto \mathrm{Q}^{-1}$.

Die Beschreibung des $\mathrm{Q}^{-1}$-Verhaltens durch Gleichung 5.5 ist aufgrund des identischen Segregationsverhaltens beider Isotope ausschließlich von der Streulängendichtedifferenz $\Delta \rho_{\mathrm{H} / \mathrm{D}}$ des jeweiligen Isotopes abhängig, die gemäß Gleichung 5.9 bestimmt werden kann. Folglich erhält man den Quotienten der absoluten Streuquerschnitte $\mathrm{d} \Sigma / \mathrm{d} \Omega$ einer deuterierten und einer mit derselben Konzentration hydrierten Probe bei festem Streuvektor Q in der dort verwendeten Notation:

$$
\frac{\left(\frac{\mathrm{d} \Sigma}{\mathrm{d} \Omega}\right)_{\mathrm{D}}}{\left(\frac{\mathrm{d} \Sigma}{\mathrm{d} \Omega}\right)_{\mathrm{H}}}=\frac{\Delta \rho_{\mathrm{D}}^{2}}{\Delta \rho_{\mathrm{H}}^{2}}=\frac{\left(\mathrm{b}_{\mathrm{D}}-\mathrm{f} \cdot \mathrm{b}_{\mathrm{Pd}} \cdot \frac{\overline{\mathrm{V}}}{\mathrm{V}_{\mathrm{Pd}}}\right)^{2}}{\left(\mathrm{~b}_{\mathrm{H}}-\mathrm{f} \cdot \mathrm{b}_{\mathrm{Pd}} \cdot \frac{\overline{\mathrm{V}}}{\mathrm{V}_{\mathrm{Pd}}}\right)^{2}}
$$

Durch einen Vergleich von Gleichung 5.10 mit experimentellen Werten kann die Ausdehnung $f$ des Segregationsgebietes bestimmt werden. Ohne radiale Ausdehnung des Segregationsgebietes $(\mathrm{f}=0)$ würde ein Wert von $\mathrm{b}_{\mathrm{D}}^{2} / \mathrm{b}_{\mathrm{H}}^{2}=3,2$ erwartet. Die Anpassungen des $\mathrm{Q}^{-1}$-Verhaltens in Abbildung 5.8 ergibt allerdings einen Wert von 1,5 für das Intensitätsver- 
hältnis. Das experimentelle Ergebnis wird gemäß Gleichung 5.10 durch eine Volumenausdehnung beschrieben, die dem 0,8-fachen des Literaturwertes für das partielle Atomvolumen $\bar{V}$ von Wasserstoff und Deuterium in Metallen entspricht.

Neben der erfolgreichen Beschreibung der Streukurven von hydrierten Proben im modifizierten Guinierplot (Kapitel 5.2.1) wurde damit ein unabhängiger experimenteller Beweis für die radiale Ausdehnung des Hydrid- bzw. Deuteridzylinders erbracht.

Der Einfluss der Deuteriumkonzentration auf das Streuverhalten der geglühten und dann zyklierten Probe wurde untersucht. Die entsprechenden Netto-Streuquerschnitte sind in Abbildung 5.9 dargestellt.

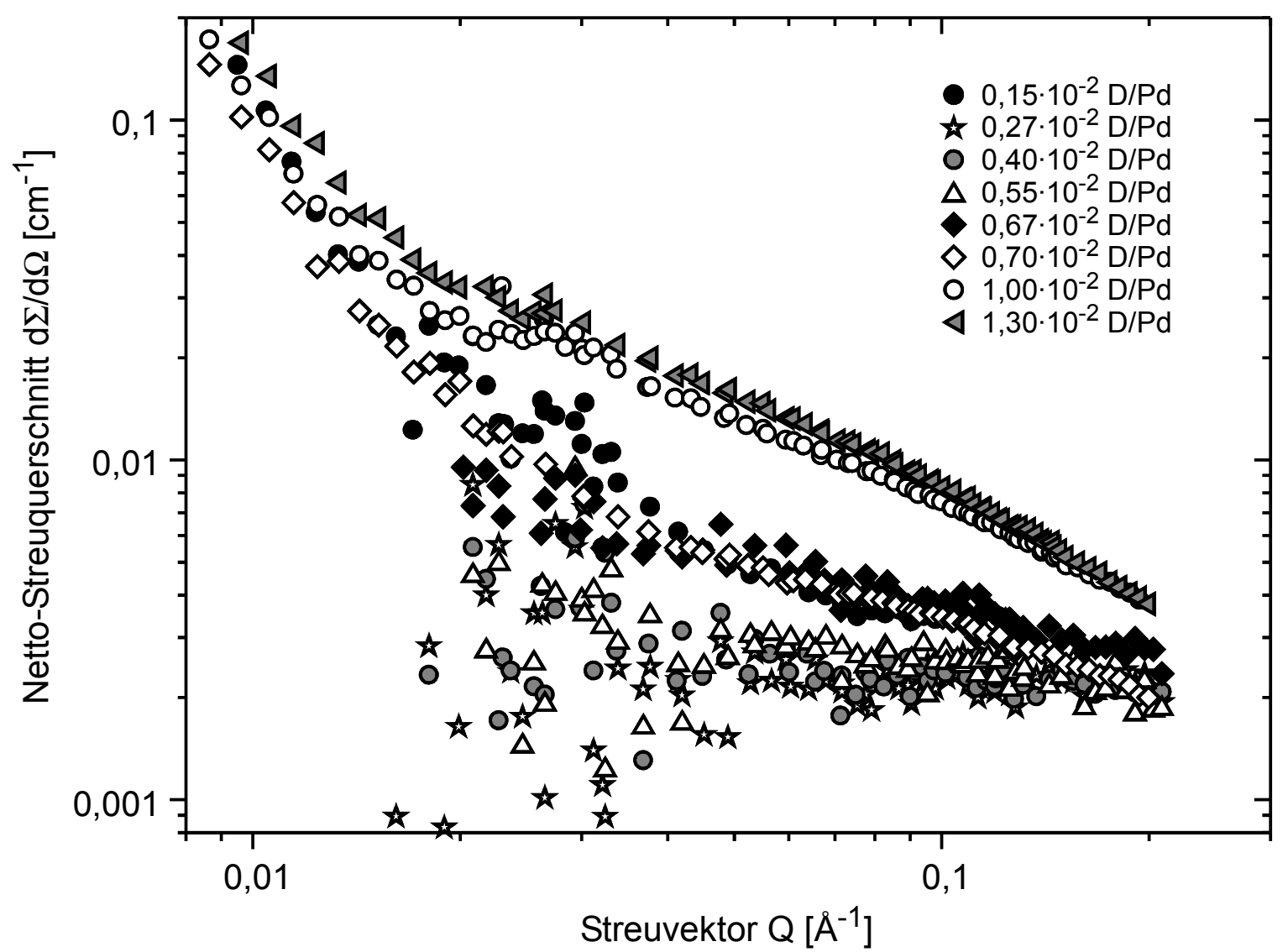

Abbildung 5.9: Übersicht der Netto-Streuquerschnitte in doppeltlogarithmischer Darstellung für die geglühte und dann zyklierte Probe mit den angegebenen Deuteriumkonzentrationen. Elektrochemische Beladungen und Messungen erfolgten bei Raumtemperatur.

Im Gegensatz zu den Streukurven der hydrierten Probe zeigte die deuterierte Probe erst bei ausreichend hohen Deuteriumkonzentrationen das erwartete modifizierte Guinierstreubild. In Kapitel 5.2.1 wurden bei der Bestimmung der Versetzungsdichte aus den Streuquerschnitten und der Ermittlung der an der Versetzungen gebunden Wasserstoffmenge jeweils ein Streu- 
beitrag vermutet, der die Streuung der Hydridzylinder verstärkt. Derselbe Beitrag sollte aufgrund des umgekehrten Vorzeichens der Streulänge für Deuterium abschwächend wirken. Es ist jedoch anzumerken, dass die Messungen der Kleinwinkelneutronenstreuung für Deuterium und Wasserstoff an unterschiedlichen Proben erfolgt sind. Für beide Proben wurde dasselbe Palladiumwalzblech elektrochemisch bei Raumtemperatur zykliert und nur im Fall der für die Messungen mit Deuterium verwendeten Probe zuvor geglüht. Offenbar führt dies zu einer unterschiedlichen Versetzungsanordnung. Der direkte Vergleich beider Proben bei derselben Deuteriumkonzentration zeigt ein unterschiedliches Streuverhalten. In Abbildung 5.10 sind die modifizierten Guinierplots der geglühten und dann zyklierten Probe bei Deuteriumkonzentrationen von $1,0 \cdot 10^{-2}$ und $1,3 \cdot 10^{-2} \mathrm{D} / \mathrm{Pd}$ dargestellt. Die Kurve zu höherer Konzentration verläuft steiler und ihrer Steigung entspricht ein Zylinderradius von 4,2 A. Außerdem enthält Abbildung 5.10 die Messwerte der gewalzten und zyklierten Probe beladen mit $1,0 \cdot 10^{-2} \mathrm{D} / \mathrm{Pd}$, denen ein Zylinderradius von 10,6 $\AA$ zuzuordnen ist.

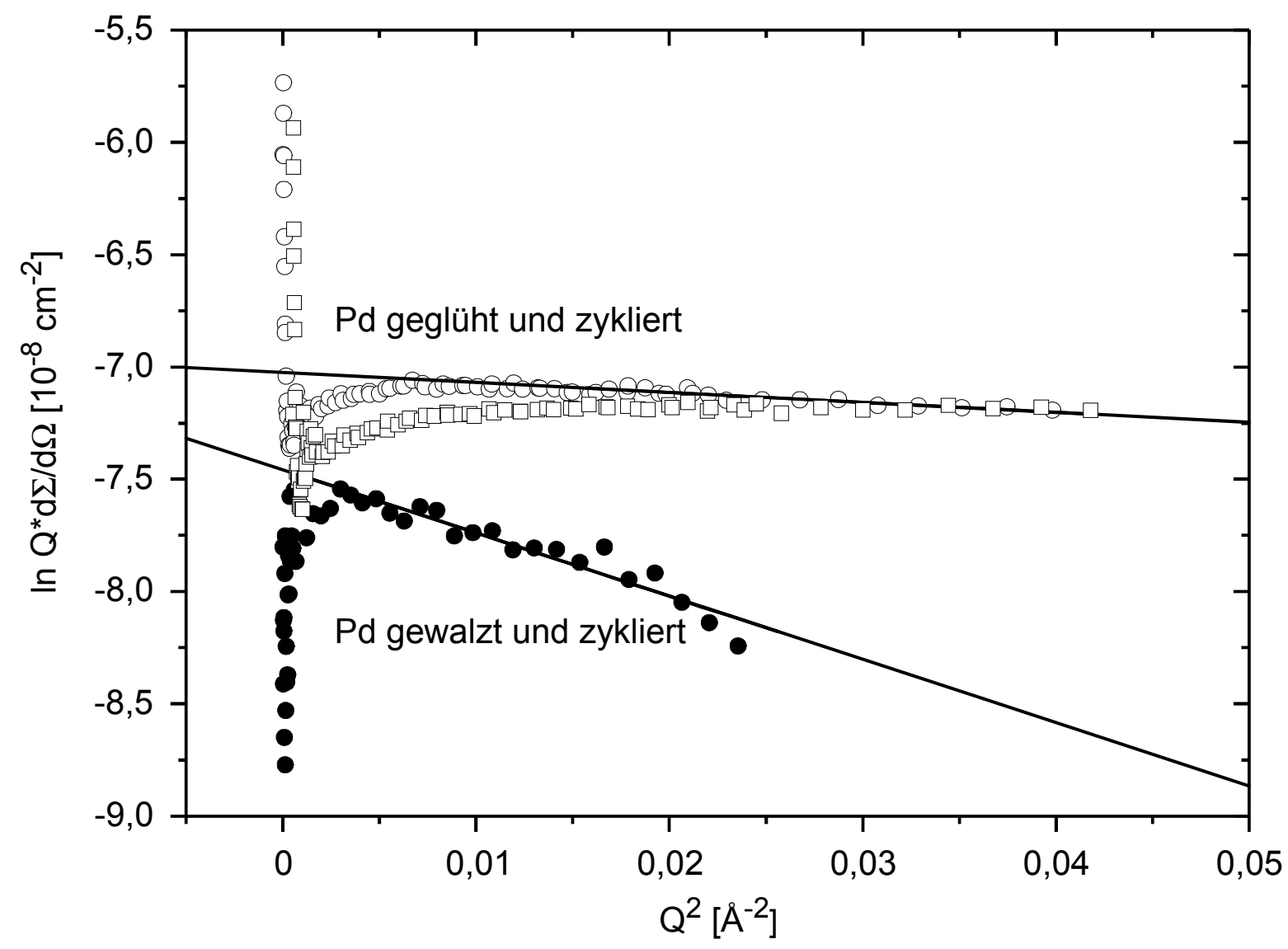

Abbildung 5.10: Modifizierter Guinierplot zweier deuterierter Proben. Die offenen Symbole beziehen sich auf die geglühte und dann zyklierte Probe beladen mit $1,0 \cdot 10^{-2} \mathrm{D} / \mathrm{Pd}$ $(\square)$ bzw. $1,3 \cdot 10^{-2} \mathrm{D} / \mathrm{Pd}(\mathrm{O})$ und die geschlossenen Kreise $(\bullet)$ auf die gewalzte und dann zyklierte Probe beladen mit $1,0 \cdot 10^{-2} \mathrm{D} / \mathrm{Pd}$. Die Geraden wurden jeweils an die durch Kreise symbolisierten Messwerte angepasst. 
Auch die Untersuchungen von Heuser und King führen in Abhängigkeit von der Probenpräparation auf Zylinderradien von 4 ...11 $\AA$ [Heu91, Heu97]. Die größten Radien wurden an Proben bestimmt, die gasvolumetrisch bei $80^{\circ} \mathrm{C}$ zykliert worden waren. Anscheindend beeinflussen die Methode des Zyklierens und die dabei eingestellte Temperatur die Defektstruktur der Probe soweit, dass sich das Streubild nachhaltig ändert.

\subsubsection{Porodverhalten}

Die Streukurven aller Palladiumproben verhielten sich bei kleinen Q-Werten wie $\mathrm{Q}^{-4}$. Eine derartige Abhängigkeit wurde in Kapitel 3.2.2 als Porodverhalten für Teilchen mit scharfen Grenzflächen und homogener Dichte durch Gleichung 3.13 beschrieben:

$$
\frac{\mathrm{d} \Sigma}{\mathrm{d} \Omega}=\frac{4 \pi \cdot \mathrm{S} \cdot(\Delta \rho)^{2}}{\mathrm{Q}^{4}}
$$

Dabei bezeichnet S die Gesamtoberfläche der Teilchen pro Einheitsvolumen.

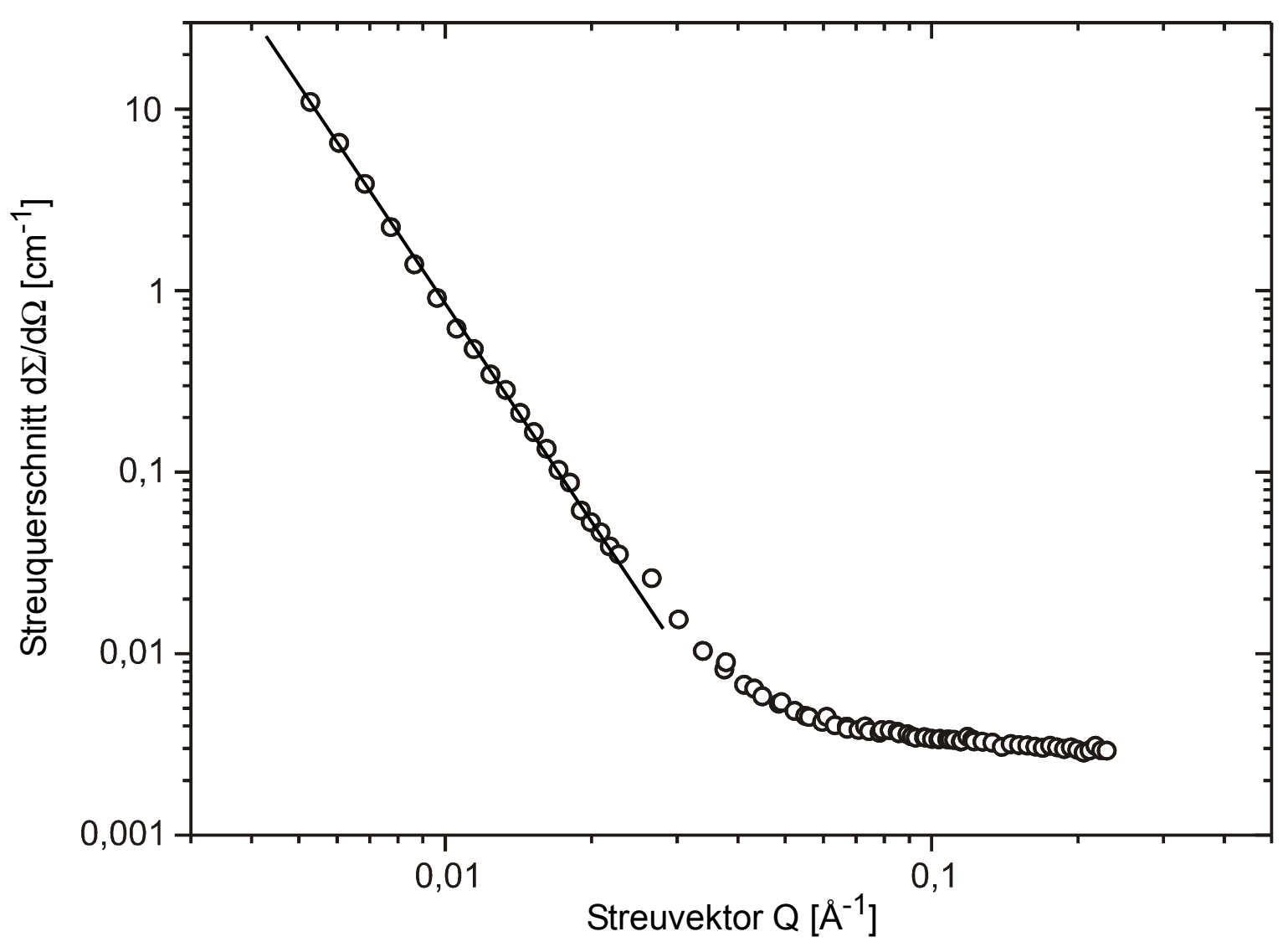

Abbildung 5.11: Absoluter Streuquerschnitt $(O)$ eines geglühten Palladiumwalzbleches in doppeltlogarithmischer Darstellung. Die Werte folgen über einen weiten Bereich des Streuvektors Q einem $\mathrm{Q}^{-4}$-Verhalten (Gerade). 
Abbildung 5.11 zeigt das Streubild einer Palladiumprobe, die jeweils zwei Stunden bei $1200{ }^{\circ} \mathrm{C}$ an Luft und bei $1150{ }^{\circ} \mathrm{C}$ im Vakuum geglüht und anschließend im Ofen abgekühlt wurde. Das Porodverhalten erstreckt sich bis $\mathrm{zu}$ dem kleinsten gemessenen Streuvektor $\mathrm{Q}=5 \cdot 10^{-3} \AA^{-1}$, so dass auf eine Teilchendimension von mindestens $200 \AA$ geschlossen wird.

Die Übereinstimmung mit anderen Autoren [Heu97, Ros99] deutet auf einen universellen Effekt für Palladium hin.

Als Ursache für das Porodverhalten werden Poren diskutiert [Ros99] und es kommen innere Oxide von Verunreinigungen durch unedlere Elemente in Frage. Die Streulängendichtedifferenz $\Delta \rho$ einer ungefüllten Pore wird durch Gleichung $5.8 \mathrm{mit}-4 \cdot 10^{10} \mathrm{~cm} / \mathrm{cm}^{-3}$ angegeben. Für Metalloxide bestimmt der Sauerstoff die Streulängendichtedifferenz und sie kann mit $\Delta \rho \approx 10^{10} \mathrm{~cm} / \mathrm{cm}^{3}$ abgeschätzt werden. Aus dem beobachteten Porodverhalten kann gemäß Gleichung 5.11 die Gesamtoberfläche S der Teilchen mit entsprechenden Streulängendichtedifferenzen bestimmt werden. Für Oxide liegt sie in der Größenordnung $10^{2} \ldots 10^{3} \mathrm{~cm}^{2} / \mathrm{cm}^{3}$ und bei Poren ergeben sich ca. $30 \mathrm{~cm}^{2} / \mathrm{cm}^{3}$ für alle Proben.

Mit der Annahme kugelförmiger Teilchen identischer Größe ist eine Abschätzung der Teilchendichte und der Verunreinigungskonzentration aus der Oberfläche S möglich. Die Teilchendichte ist mit $10^{12} \ldots 10^{14} \mathrm{~cm}^{-3}$ so klein, dass ein direkter Nachweis durch Elektronenmikroskopie unwahrscheinlich ist. Das beobachtete Porodverhalten wird durch Verunreinigungskonzentrationen von $10^{-4}$ erklärt, die in den Proben nachgewiesen wurden (Tabelle 4.1).

Innere Oxide sind bekannt als Haftstellen für Wasserstoff [Geg97] und erklären daher die Kontrastvariation aufgrund der Dekoration mit Wasserstoff bzw. Deuterium, wie sie beispielsweise in Abbildung 5.8 bei kleinen Werten des Streuvektors Q beobachtet wird. 


\section{Zusammenfassung}

In der vorliegenden Arbeit wurde das Segregationsverhalten von Wasserstoff und Deuterium an Versetzungen in Palladium untersucht. Die Charakterisierung der räumlichen Ausdehnung des Segregationsgebietes erfolgte dabei mit der Methode der Kleinwinkelneutronenstreuung. Gasvolumetrische Messungen der Löslichkeit wurden zur Bestimmung der segregierten Stoffmenge eingesetzt.

Die Netto-Streukurven, d. h. die Differenz der Streukurven einer Probe im beladenen und unbeladenen Zustand, folgen sowohl für Wasserstoff als auch für Deuterium in einem großen Bereich des Streuvektors $Q$ einem $Q^{-1}$-Verhalten. Für linienhafte Streuobjekte mit einem gegenüber der Länge $2 \mathrm{H}$ kleinen Durchmesser 2R wird dieses Verhalten bei Werten des Streuvektors $\mathrm{Q}$ erwartet, für die $\mathrm{HQ} \gg 1$ und $\mathrm{RQ} \ll 1$ gilt. Die Segregationsgebiete erfüllen demnach diese Bedingung und stellen daher linienhafte Teilchen dar.

Das Streuverhalten des Segregationsgebietes wurde in dieser Arbeit erstmalig auch für Wasserstoff untersucht. Die Streukurven zeigen bei größeren Streuvektoren eine Abweichung vom oben beschriebenen $\mathrm{Q}^{-1}$-Verhalten, die auf den Einfluss der radialen Ausdehnung des Segregationsgebietes zurückgeführt wird. Der Vergleich der Streukurven mit dem Streumodell eines Zylinders im modifizierten Guinierplot ergab eine Abhängigkeit der Zylinderradien von der Wasserstoffkonzentration. Die Radien nehmen in der $\alpha$-Phase des Systems PalladiumWasserstoff von ca. $5 \AA$ auf etwa $13 \AA$ mit der Konzentration zu. Das Ausmaß der Segregation ist damit größer als aufgrund einer rein elastischen Wechselwirkung des Wasserstoffes mit dem Stufenanteil der Versetzungen zu erwarten wäre. Entsprechende theoretische Rechnungen von Ross und Stefanopoulos ergeben innerhalb der hier untersuchten $\alpha$-Phase deutlich kleinere Ausdehnungen des Segregationsgebietes von 2 ... $5 \AA$ [Ros94]. Die Beschreibung der konzentrationsabhängig experimentell gewonnen Zylinderradien gelang in dieser Arbeit mit einem thermodynamischen Modell, welches eine direkte HH- bzw. DD-Wechselwirkung vergleichbar der Hydridbildungsenthalpie berücksichtigt. Die Segregationsgebiete können also als tangential zur Versetzungslinie orientierte Zylinder verstanden werden, in denen die Konzentration vermutlich der des Hydrids bzw. Deuterids entspricht.

Die Ausdehnung des Hydrid- bzw. Deuteridzylinders innerhalb der Palladiummatrix konnte außerdem durch einen Vergleich der Streuintensitäten von Wasserstoff und Deuterium, deren unterschiedliche Streulängen zu einer Kontrastvariation führen, im Bereich der Zylinderstreuung an einer identischen Probe bestätigt werden. Das experimentelle Ergebnis wird durch eine Volumenausdehnung beschrieben, die $80 \%$ des Literaturwertes für das partielle Atomvolumen von Wasserstoff und Deuterium in Metallen beträgt.

Die an den Versetzungen gebundene Wasserstoff- bzw Deuteriummenge konnte gasvolumetrisch gemessen werden. Für beide Isotope ist die Löslichkeitserhöhung identisch. Der Faktor 
nimmt innerhalb der $\alpha$-Phase ab und beträgt 1,2 bei $5 \cdot 10^{-3} \mathrm{H} / \mathrm{Pd}$. Die Löslichkeitserhöhung stimmt gut mit den Ergebnissen der Neutronenstreuung überein, aus denen ebenfalls die segregierte Stoffmenge unter Verwendung der elektronenmikroskopisch erhaltenen Versetzungsdichte berechnet wurde. Die Annahme eines Hydrid- bzw. Deuteridzylinders wird damit bestätigt.

Das Streuverhalten der deuterierten Proben unterschied sich von dem der hydrierten auch im modifizierten Guinierplot. Da die Segregation von Wasserstoff und Deuterium nach den Ergebnissen der Gasvolumetrie identisch ist, scheint der Zylinderstreuung ein weiterer Streubeitrag überlagert sein, der bei Deuterierung abschwächend und bei Hydrierung verstärkend wirkt. Unterschiede zwischen gewalzten und zyklierten Proben fanden auch Heuser und King [Heu97]. Derartige Effekte könnten durch Versetzungsagglomerate und Leerstellencluster verursacht werden, die von der Probenpräparation abhängen.

Die SANS-Streukurven aller Palladiumproben zeigten ein meist ausgeprägtes Porodverhalten, welches auf Teilchen der Größenordnung $100 \AA \AA$ schließen läßt. Die Übereinstimmung mit anderen Autoren [Heu97, Ros99] deutet auf einen universellen Effekt für Palladium hin, der durch Poren oder Fremdatomoxide erklärt werden kann. Die anzunehmende Konzentration von $10^{-4}$ stimmt gut mit der Verunreinigungskonzentration vieler unedlerer Elemente in Palladium überein. Es ist bekannt, dass Metall-Metalloxidgrenzflächen attraktive Haftstellen für Wasserstoff darstellen. Das Porodverhalten und seine Kontrastvariation durch die Dekoration mit Wasserstoff bzw. Deuterium werden daher wahrscheinlich durch Oxide verursacht.

In dieser Arbeit ist der direkte Nachweis von Hydridzylindern tangential zur Versetzungslinie mit Kleinwinkelneutronenstreuung gelungen und die Abhängigkeit der radialen Ausdehnung von der Wasserstoffkonzentration konnte mit einem thermodynamischen Modell beschrieben werden. Die für beide Isotope identische Löslichkeitserhöhung aufgrund von Versetzungen deutet ebenso wie das jeweils beobachtete Streuverhalten linienhafter Teilchen auf ein analoges Segregationsverhalten hin. Ein unabhängiger Nachweis der Ausdehnung des Segregationszylinders innerhalb der Palladiummatrix gelingt daher durch Kontrastvariation zwischen Wasserstoff und Deuterium. 


\section{Literaturverzeichnis}

[Abe62] P. Abens und W. G. Burgers, Trans. Farad. Soc., 58 (1962) 1989

[And96] C. Anderton, N. Strother, J. Pote, R. Foley, K. Rebeiz, S. Nesbit, A. Craft, Scripta Met., 35 (1996) 1013

[Atk58] H. H. Atkinson, P. B. Hirsch, Phil. Mag., 3 (1958) 213

[Bas98] M. I. Baskes, R. G. Hoagland, SAND98-8500 (1998) to be published

[Bes58] D. N. Beshers, Acta Metall., 6 (1958) 521

[Bla99] D. Blavette, E. Cadel, A. Fraczkiewicz, A. Menand, Science, 286 (1999) 2317

[Bou82] A. Bourret, C. Colliex, Ultramicroscopy, 9 (1982) 183

[Bro72] H. Brodowsky, Ber. Bunsenges. Physik. Chem., 76 (1972) 740

[Cle73] J. D. Clewley, T. Curran, T. B. Flanagan, W. A. Oates, Trans. Faraday Soc., 73 (1973) 449

[Cot49] A. H. Cottrell, B. A. Bilby, Proc. Phys, Soc. Lond., A62 (1949) 49

[Cot64] A. H. Cottrell, ,, Theory of Crystal Dislocations “, Documents in Modern Physics, Gordon and Breach, New York (1964)

[Dre76] W. Drexel, A. Murani, D. Tocchetti, W. Kley, I. Sosnowska, D. K. Ross, J. Phys. Chem. Sol., 37 (1976) 1135

[Fi193] A. P. Filippov, G. N. Gaidukov, Phil. Mag. A, 67 (1993) 109

[Fla76a] T. B. Flanagan, J. F. Lynch, J. D. Clewley, B. von Turkovich, J. less-common metals, 49 (1976) 13

[Fla76b] T. B. Flanagan, J. F. Lynch, J. Less Comm. Met., 49 (1976) 25

[Fla87] T. B. Flanagan, J. of the Korean Inst. of Metals, 25 (1987) 41

[Fla91] T. B. Flanagan und W. A. Oates, Ann. Rev. Mater: Sc., 21 (1991) 269

[Gla82] Small angle X-ray scattering, herausgegeben von O. Glatter, O. Kratky, Acad. Pr., London (1982)

[Geg97] J. Gegner, G. Hörz, R. Kirchheim, Interface Science, 5 (1997) 231

[Gra66] T. Graham, Phil. Trans. Roy. Soc. London, 156 (1866) 399

[Gri97] R. Griessen, J. N. Huiberts, M. Kremers, A. T. M. van Gogh, N. J. Koeman, J. P. Dekker, P. H. L. Notten, J. of alloys and compounds, 253 (1997) 44

[Gui63] A. Guinier, X-Ray Diffraction, Freeman, San Francisco (1963)

[Heu91] B. J. Heuser, J. S. King, G. C. Summerfield, F. Boué, J. E. Epperson, acta metall. mater., 11 (1991) 2815

[Heu97] B. J. Heuser, J. S. King, J. of Alloys and Compounds, 261 (1997) 225

[Hir68] J. P. Hirth, J. Lothe, ,, Theory of Dislocations “, MC. Graw-Hill, New York (1968)

[Jam76] H. C. Jamieson, G. C. Weatherly, F. D. Manchester, J. Less. Comm. Met., 50 (1976) 85

[Kir81a] R. Kirchheim, Acta Metall., 29 (1981) 835

[Kir81b] R. Kirchheim, Acta Metall., 29 (1981) 845 
[Kir81c] R. Kirchheim, Proc. TMS-AIME Fall Meeting, Louisville, Kentucky/USA

[Kir82] R. Kirchheim, Acta metall., 30 (1982) 1069

[Kir83] R. Kirchheim, J. Non-Crystalline Solids, 55 (1983) 243

[Kir88] R. Kirchheim, Prog. Mater. Sci., 32 (1988) 261

[Kir98] R. Kirchheim, persönliche Mitteilung

[Kos93] G. Kostorz, Physica Scripta, T49 (1993) 636

[Li66] J. C. M. Li, R. A. Oriani, L. S. Darken, Z. Phys. Chem., 49 (1966) 271

[Lyn91] F. E. Lynch, J. less comm. Met., 172 (1991) 943

[Mak80] B. J. Makenas, H. K. Birnbaum, Acta Metall., 28 (1980) 979

[Nør80] J. K. Nørskov, N. D. Lang, Phys. Rev., B21 (1980) 2131

[Nor86] P. Nordlander, J. K. Nørskov, F. Besenbacher, J. Phys., F16 (1986) 1161

[Oat81]～W. A. Oates und T. B. Flanagan, Proc. Sol. St. Chem., 13 (1981) 193

[Pei78] H. Peisl, in „Hydrogen in MetalsI“, herausgegeben von G. Alefeld und J. Völkl, Topics in Applied Physics, Vol. 28, Springer Verlag, Berlin (1978) 53

[Por51] G. Porod, Kolloid Z., 124 (1951) 83

[Por52] G. Porod, Kolloid Z., 125 (1952) 51

[Pyc91] W. Pyckhout-Hintzen, T. Springer, F. Forster, W. Gronski, C. Frischkorn, Macromolecules, 24 (1991) 1269

[Rau76] E. Raub, in „, Gase und Kohlenstoff in Metallen “, herausgegeben von E. Fromm und E. Gebhardt, Reine und angewandte Metallkunde in Einzeldarstellungen, Band 26, Springer-Verlag, Berlin (1976) 636

[Rod83] J. A. Rodrigues, R. Kirchheim, Scripta Met., 17 (1983) 159

[Ros94] D. K. Ross, K. Stefanopoulos, Z. Phys. Chem., 183 (1994) 29

[Ros99] D. K. Ross, K. Stefanopoulos, M. Kemali, J. Alloys and Compounds, 293-295 (1999) 346

[Rus84] J. J. Rush, J. M. Rowe, D. Richter, Z. Phys., 10 (1984) 283

[Rus85] J. J. Rush, J. M. Rowe, D. Richter, Z. Phys. B: Condensed Matter, 55 (1985) 283

[Sea92] V.F. Sears, Neutron News, 3 (1992) 26-37

[Sch82] H. E. Schaefer, Positron Annihilation, herausgegeben von P. G. Coleman, S. C. Sharma, L. M. Diana, North Holland Publish Company (1982)

[Sie29] A. Sieverts, Zeitschrift f. Metallkunde, 2 (1929) 37

[Szö87] A. Szökefalvi-Nagy, H. Y. Huang, R. Kirchheim, J. Phys. F, 17 (1987) 427

[Vö178] J. Völkl, G. Alefeld in „Hydrogen in MetalsI“, herausgegeben von G. Alefeld und J. Völkl, Topics in Applied Physics, Vol. 28, Springer Verlag, Berlin (1978)

[Wic78] E. Wicke und H. Brodowsky in Zusammenarbeit mit H. Züchner, in ,,Hydrogen in Metals II", herausgegeben von G. Alefeld und J. Völkl, Topics in Applied Physics, Vol. 29, Springer Verlag, Berlin (1978) 73

[Wig87] G. D. Wignall, F. S. Bates, J. Appl. Cryst., 20 (1987) 28 


\section{Lebenslauf}

\section{Persönliche Daten}

Name: $\quad$ Michael Maxelon

Geburtsort: Kassel

Geburtsdatum: 16. Januar 1970

Nationalität: deutsch

\section{Ausbildung}

Schulbildung

1989 Allgemeine Hochschulreife

Engelsburg-Gymnasium Kassel

Wehrdienst

6/89 bis $8 / 90$ Grundwehrdienst

Flugabwehrregiment 2 Kassel

\section{Studium}

9/90 Diplomstudiengang Physik

an der Georg-August-Universität Göttingen

10/92 Diplomvorprüfung Physik

4/94 bis 12/95 Diplomarbeit am Institut für Metallphysik über „TEM- und DSC-Untersuchungen der mechanischen Entordnung von $\mathrm{Cu}_{3} \mathrm{Au}^{\text {“ }}$

2/96 Diplomprüfung im Fach Physik

seit 4/96 Wissenschaftlicher Mitarbeiter am Institut für Materialphysik der Georg-August-Universität Göttingen in der Arbeitsgruppe von Prof. Dr. R. Kirchheim

seit 10/97 Zusatzstudiengang Wirtschaftswissenschaften an der Georg-August-Universität Göttingen 